\title{
Distributed Learning-based Cooperative Spectrum Sensing for Cognitive Internet of Things Systems
}

by

\author{
Anastassia Gharib, B.Sc. \\ A thesis submitted to the \\ Faculty of Graduate and Postdoctoral Affairs \\ in partial fulfillment of the requirements for the degree of
}

Master of Applied Science in Electrical and Computer Engineering

Ottawa-Carleton Institute for Electrical and Computer Engineering

Department of Systems and Computer Engineering

Carleton University

Ottawa, Ontario

April, 2018

(C) Copyright

Anastassia Gharib, 2018 


\section{Abstract}

The emerge of Internet of Things (IoT) brings up revolutionary changes to the field of wireless communications. Providing connection to billions of IoT devices together with challenging IoT environments lead to the need of new technologies in order to fulfill IoT spectrum demands. Cognitive radio (CR) technology can be seen as one of the prominent solutions to the spectrum scarcity issue in IoT, where multiband cooperative spectrum sensing (CSS) is the key. In this thesis, we consider heterogeneous distributed learning-based CR networks (CRNs). Focus is given to learning approaches named as incremental, consensus, and diffusion. We perform a comparative study to investigate which one of them best fits cognitive IoT demands. Simulation results are performed illustrating potentials of diffusion and consensus algorithms and disadvantages of incremental for cognitive IoT systems.

Lack of centralized control, increase in number of devices, and dynamic environments place a room for lots of challenges in the CSS process. Conventional CSS techniques have to be improved in order to fulfill sophisticated IoT requirements. One of the main challenges is cooperative secondary users' (SUs') scheduling to sense a subset of channels. To overcome the aforementioned challenge, in this thesis, we propose a novel multi-band CSS scheme, named as heterogeneous multi-band multi-user CSS (HM2CSS). The proposed scheme allows heterogeneous SUs to sense multiple channels and works by selecting cooperative SUs in two stages. Only SUs owning different information about channels are chosen to be cooperative. This is done by selecting leaders for each channel in the first stage and corresponding cooperative SUs in the second stage. Careful choice of leaders reflects the selection of cooperative SUs and hence, improves system performance. Then, diffusion learning algorithm is used to exchange locally sensed information among cooperative SUs for all channels. Further, the decision on the availability of channels is made.

Extensive simulation results illustrate that the proposed HM2CSS scheme satisfies the IEEE 802.22 detection performance standard. Detection performance and CRN 
throughput results are improved compared to the existing multi-band CSS schemes. Furthermore, it is shown that the proposed HM2CSS scheme is scalable in terms of detection performance. This allows new nodes to join the CRN without additional human intervention to bring the performance level back. Compared to the existing multi-band CSS schemes, system's detection performance does not degrade when increasing the CRN's size. In addition, the proposed HM2CSS scheme provides fair energy consumption for CSS on all channels and illustrates lower aggregate energy consumption for the system in total. Nevertheless, HM2CSS has its own drawbacks yet to overcome. It introduces higher computational complexity and power consumption per channel for certain detector types. Finally, future research insights are given in order to improve and overcome the drawbacks of HM2CSS and existing multi-band CSS schemes. 


\section{List of Publications}

- Oral presentation, Scalable Learning-based Cooperative Spectrum Sensing for Distributed IoT Systems, Annual IoT613 Conference, April 20, 2018.

- A. Gharib, W.Ejaz, and M. Ibnkahla, Heterogeneous Multi-band Multi-user Cooperative Spectrum Sensing for Spectrum Analytics in IoT Systems, Data Day 5.0, Carleton University, June 5, 2018.

- Z. Bouida, A. Gharib, and M. Ibnkahla, Secondary Systems Scheduling Using Precoding-Aided Space Shift Keying for Overlay Cognitive Radio, IEEE Wireless Communications and Networking Conference (WCNC), Barcelona, Spain, pp. 1-5, April 2018.

- A. Gharib, W. Ejaz, and M. Ibnkahla, Distributed Learning-based Multi-band Multi-user Cooperative Sensing in Cognitive Radio Networks, submitted to IEEE Global Communications Conference (GLOBECOM), 2018.

- A. Gharib, W. Ejaz, and M. Ibnkahla, Learning-based Multi-Band Spectrum Sharing for IoT in Next Generation Networks, to be submitted to IEEE Communications Magazine, 2018.

- A. Gharib, W. Ejaz, and M. Ibnkahla, Scalable Learning-based Heterogeneous Multi-band Multi-user Cooperative Spectrum Sensing for Distributed IoT Systems, to be submitted to IEEE Internet of Things Journal, 2018. 
To my beloved family 


\section{Acknowledgments}

My stay at Carleton University has helped me grow excessively not only as a researcher, but also as an individual. I would like to thank many people for being an amazing part of my life throughout the years of study.

To begin with, I would like to express my special thanks of gratitude to my supervisor, Prof. Mohamed Ibnkahla, who gave me the golden opportunity to become a part of the Sensor Systems and Internet of Things laboratory, providing me with unfailing support and continuous encouragement. I will always be grateful for his tremendous favors and kindness. In addition to this, I am using this opportunity to extend my warm thanks to Dr. Waleed Ejaz for his guidance. I am grateful to find someone as professional as he is. I would also like to thank Dr. Zied Bouida for creating a comfortable working environment of Sensor Systems and Internet of Things laboratory, as well as I am gratefully indebted for his valuable comments. I would like to show great appreciation to my dear friend, Asha Siad, for dedicating time to proofread this thesis.

Moreover, I am sending my special thanks to Eng. Loai Tamimi and Prof. Nihad Dib for their influence on me. Indeed their support and insistence has guided me towards the road of starting graduate studies in Canada.

A special part of my heart is devoted to my family. I would like to express very profound gratitude to my parents for accepting nothing less than excellence from me throughout my life, constant care, love, and patience. Last but not least, I would like to thank my little sisters for supporting me spiritually throughout writing this thesis and my life in general, being my best friends. This accomplishment would not have been possible without my beloved family. Thank you. 


\section{Table of Contents}

Abstract $\quad$ ii

List of Publications $\quad$ iv

Acknowledgments $\quad$ vi

Table of Contents vii

List of Tables $\quad$ x

List of Figures $\quad$ xi

Nomenclature $\quad$ xiv

1 Introduction 1

1.1 Motivation . . . . . . . . . . . . . . . . . 2

1.2 Problem Formulation . . . . . . . . . . . . . . . . . 2

1.3 Thesis Outline and Contributions . . . . . . . . . . . . . 3

1.3.1 Thesis Outline. . . . . . . . . . . . . . . 3

1.3.2 Main Contributions ................. 4

1.4 Notations . . . . . . . . . . . . . . . . . . . 5

2 Background $\quad 6$

2.1 Introduction . . . . . . . . . . . . . . . . . . 6

2.1.1 Internet of Things Concept . . . . . . . . . . . . . . . 6

2.1.2 Cognitive Radio Overview . . . . . . . . . . . . . . . . 8

2.1.3 Incorporating Cognitive Radio into Internet of Things . . . . 11

2.2 The Multi-band Cooperative Spectrum Sensing Problem . . . . . . . 13

2.3 Related Work . . . . . . . . . . . . . . . . . . . . . 15 
2.4 Performance Measures . . . . . . . . . . . . . . . . . . . . . . 16

2.4.1 Detection Performance . . . . . . . . . . . . . . . 16

2.4.2 Cognitive Radio Network Throughput _. . . . . . . . . . 21

2.4.3 Energy Consumption on the Sensing Process . . . . . . . . . 23

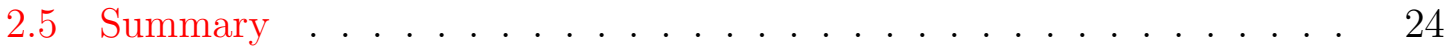

3 Cognitive Internet of Things Systems 25

3.1 Introduction . . . . . . . . . . . . . . . . . . . . . 25

3.2 IoT-based System Architecture _ . . . . . . . . . . . . . 25

3.2.1 Centralized Cognitive IoT Network Architecture . . . . . . . 26

3.2.2 Distributed Cognitive IoT Network Architecture . . . . . . . 26

3.2.3 Hybrid Cognitive IoT Network Architecture . . . . . . . . . 28

3.3 Cooperative Spectrum Sensing Challenges in Cognitive IoT Systems . 28

3.3.1 Application-Centric Approach / Treatment . . . . . . . . . . 29

3.3.2 Mobility of Users . . . . . . . . . . . . . . . . . . . . 29

3.3.3 Scalability, High Integrity, and Heterogeneity . . . . . . . . . 29

3.3.4 Cooperation and Learning . . . . . . . . . . . . . . . . 29

3.3.5 Maintaining Energy Efficiency . . . . . . . . . . . . . . 30

3.3.6 Fairness and Cooperative Secondary IoT-nodes Selection . . . 30

3.3.7 Computational Complexity . . . . . . . . . . . . . . . 31

3.3.8 Sensing Duration and Synchronization . . . . . . . . . 31

3.3 .9 Interference Management . . . . . . . . . . . . . . . 31

3.4 Summary . . . . . . . . . . . . . . . . . . . . 32

4 Distributed Learning Approaches 33

4.1 Introduction . . . . . . . . . . . . . . . 33

4.2 Centralized Rules / Algorithms . . . . . . . . . . . . . . 34

4.3 Distributed Learning Algorithms . . . . . . . . . . . . . 35

4.3.1 Incremental-based Approach . . . . . . . . . . . . . . . 35

4.3.2 Consensus-based Approach . . . . . . . . . . . . . . . . 36

4.3.3 Diffusion-based Approach . . . . . . . . . . . . . . . 36

4.3 .4 Comparison . . . . . . . . . . . . . . . . . 38

4.4 Simulation Results . . . . . . . . . . . . . . . . . . . . 40

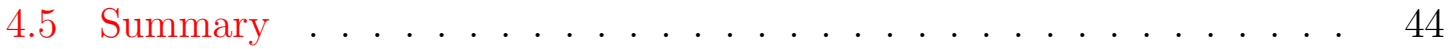


5 Heterogeneous Multi-band Multi-user CSS 46

5.1 Introduction . . . . . . . . . . . . . . . . 46

5.2 System Model . . . . . . . . . . . . . . . . . . . . 47

5.3 Heterogeneous Multi-band Multi-user Cooperative Spectrum Sensing 48

5.3.1 Stage 1: Leader Selection . . . . . . . . . . . . . . . . 50

5.3.2 Stage 2: Cooperative Secondary User Selection . . . . . . . . . 53

5.4 Simulation Results . . . . . . . . . . . . . . . . . . . 56

5.4.1 Diffusion Learning Analysis . . . . . . . . . . . . . . . . . 58

5.4.2 Receiver Operating Characteristic Results . . . . . . . . . . . 61

5.4.3 Aggregate Primary User Protection Level vs Average Signalto-Noise Ration Analysis . . . . . . . . . . . . . . . . . . . . 67

5.4.4 Throughput Analysis . . . . . . . . . . . . . . . 70

5.4.5 Energy Consumption on the Sensing Process . . . . . . . . . . 73

5.5 Summary . . . . . . . . . . . . . . . . 75

6 Conclusions and Future Work $\quad 77$

6.1 Conclusions . . . . . . . . . . . . . . . . . . 77

6.2 Future Work . . . . . . . . . . . . . . . . . . . . . 79

$\begin{array}{ll}\text { List of References } & 82\end{array}$ 


\section{List of Tables}

4.1 Comparison of incremental, consensus, and diffusion-based learning approaches' characteristics. . . . . . . . . . . . . . . . . . . 37

4.2 Comparison of incremental, consensus, and diffusion-based learning approaches' complexity on sensing the $m$-th channel per one iteration.

5.1 Number of channels with the certain information available about them for the total of $M$ channels. . . . . . . . . . . . . . . . . . 


\section{List of Figures}

2.1 Expected growth in the number of devices connected to the Internet [11]. 7

2.2 Major application domains of IoT. . . . . . . . . . . . . . 8

2.3 The CR cycle overview. . . . . . . . . . . . . . . . . . . . . . . . 9

2.4 The CR paradigms: opportunistic spectrum access, underlay, and overlay. . . . . . . . . . . . . . . . . . . . . 10

2.5 IoT-based CR cycle. . . . . . . . . . . . . . . . . . 11

2.6 A flow chart for multi-band CSS. . . . . . . . . . . . . . . 14

2.7 Block diagram of the ED-based case. . . . . . . . . . . . . . . 18

2.8 Block diagram of the PD-based case. . . . . . . . . . . . . . . . . 19

2.9 Block diagram of the OD-based case. . . . . . . . . . . . . . 20

2.10 Detection performance overview for PD, OD, and ED-based scenarios. 22

2.11 The structure of one frame for CSS. . . . . . . . . . . . . . . 23

3.1 Heterogeneous secondary IoT-based networks: (a) centralized, (b) distributed, and (c) hybrid. . . . . . . . . . . . . . . . 27

4.1 Homogeneous distributed ED-based topology. . . . . . . . . . . . . . 39

4.2 Incremental-based learning curves of five cooperative secondary EDbased IoT-nodes sensing channel 2: (a) without the link failure and (b) with the $30 \%$ link failure. . . . . . . . . . . . . . . . 40

4.3 Consensus-based learning curves of five cooperative secondary EDbased IoT-nodes sensing channel 2: (a) without the link failure and (b) with the $30 \%$ link failure. . . . . . . . . . . . . . .

4.4 Diffusion-based learning curves of five cooperative secondary ED-based IoT-nodes sensing channel 2: (a) without the link failure and (b) with the $30 \%$ link failure. . . . . . . . . . . . . . . . 
4.5 Scalability results, average number of iterations needed to reach a consensus point for different values of cooperative secondary IoT-nodes using (a) incremental, (b) consensus, and (c) diffusion-based learning algorithms. . . . . . . . . . . . . . . . . . 43

5.1 Heterogeneous distributed multi-user CRN topology. . . . . . . . . . 47

5.2 A flow chart for the proposed HM2CSS scheme. . . . . . . . . . . . . 49

5.3 The diffusion learning process illustration of the ED-based channel 2 for: (a) the proposed HM2CSS scheme and (b) the existing RSSS scheme. 58

5.4 The diffusion learning process illustration of the PD-based channel 3 for: (a) the proposed HM2CSS scheme and (b) the existing RSSS scheme. 59

5.5 The diffusion learning process illustration of the OD-based channel 5 for: (c) the proposed HM2CSS scheme and (d) the existing RSSS scheme. 60

5.6 Global ROC results for the proposed distributed HM2CSS scheme compared to the existing distributed RSSS scheme for different network sizes. 61

5.7 Global ROC results with various maximum number of channels allowed to be sensed by one SU for both the proposed distributed HM2CSS scheme and the existing distributed RSSS scheme. . . . . . . . . .

5.8 Global ROC results for the proposed distributed HM2CSS scheme compared to the existing centralized schemes. . . . . . . . . . . . . .

5.9 Global ROC results for the fixed and variable number of samples between cooperative SU-clusters of the proposed distributed HM2CSS scheme. . . . . . . . . . . . . . . . . .

5.10 Global PU protection level vs $S N R_{\text {avg }}$ results for the proposed distributed HM2CSS scheme compared to the existing distributed RSSS scheme for different network sizes. . . . . . . . . . . . . . . . .

5.11 Global PU protection level vs $S N R_{\text {avg }}$ results for different values of maximum number of channels sensed by one SU for the proposed distributed HM2CSS scheme and the existing distributed RSSS scheme.

5.12 Global PU protection level vs $S N R_{\text {avg }}$ results for the proposed distributed HM2CSS scheme compared to the existing centralized schemes. 69

5.13 Global PU protection level vs $S N R_{\text {avg }}$ results for different values of global false alarm probability being fixed for the proposed HM2CSS scheme. . . . . . . . . . . . . . . . . . . 70 
5.14 Aggregate CRN throughput results for the proposed distributed HM2CSS scheme and the existing distributed RSSS scheme. . . . . . 71

5.15 Aggregate CRN throughput results for the proposed distributed HM2CSS scheme and the existing centralized schemes. . . . . . . . . 72

5.16 Comparison of the proposed HM2CSS scheme with the existing RSSS scheme in terms of average energy consumption level across each: (a) ED-sensed channel, (b) PD-sensed channel, and (c) OD-sensed channel. 73

5.17 Comparison of proposed HM2CSS with existing RSSS in terms of average sensing energy consumption level across similar channel types. . 74

5.18 Aggregate energy consumption level on spectrum sensing for proposed HM2CSS and existing RSSS. . . . . . . . . . . . . . . 75 


\title{
Nomenclature
}

\author{
Notation \\ Description \\ AWGN \\ Additive White Gaussian Noise \\ BPSK \\ Binary Phase Shift Keying \\ $\mathrm{B} \& \mathrm{~B}$ \\ Branch and Bound \\ CDF \\ Cumulative Distribution Function \\ $\mathrm{CE}$ \\ Cost Efficiency \\ CR \\ Cognitive Radio \\ CRN \\ Cognitive Radio Network \\ CSS \\ Cooperative Spectrum Sensing \\ CVM \\ Cramer-Von Mises \\ ED \\ Energy Detector \\ EE \\ Energy Efficiency \\ $\mathrm{EH}$ \\ Energy Harvesting \\ FC \\ Fusion Center \\ HM2CSS \\ Heterogeneous Multi-band Multi-user Cooperative Spectrum Sensing \\ IID \\ Independent and Identically Distributed \\ IoT \\ Internet of Things \\ MILP \\ Mixed Integer Linear Programming




$\begin{array}{ll}\text { NGN } & \text { Next Generation Network } \\ \text { OD } & \text { Orthogonal frequency division multiplexing-based Detector } \\ \text { OFDM } & \text { Orthogonal Frequency Division Multiplexing } \\ \text { PD } & \text { Pilot tone-based Detector } \\ \text { PN } & \text { Primary Network } \\ \text { PSK } & \text { Phase Shift Keying } \\ \text { PSSS } & \text { Persistent Spectrum Sensing Strategy } \\ \text { PU } & \text { Primary User } \\ \text { QAM } & \text { Quadrature Amplitude Modulation } \\ \text { QoS } & \text { Quality of Service } \\ \text { RFEH } & \text { Radio Frequency Energy Harvesting } \\ \text { ROC } & \text { Receiver Operating Characteristics } \\ \text { RSSS } & \text { Random Spectrum Sensing Strategy } \\ \text { RV } & \text { Random Variable } \\ \text { SE } & \text { Spectral Efficiency } \\ \text { SINR } & \text { Signal-to-Interference-and-Noise Ratio } \\ \text { SNR } & \text { Signal-to-Noise Ratio } \\ \text { SU } & \text { Secondary User }\end{array}$




\section{Chapter 1}

\section{Introduction}

In heterogeneous Internet of Things (IoT) systems, spectrum scarcity is believed to be one of the most crucial problems [1,2]. Supporting the large number of IoT devices' pervasive connectivity is a hard task to achieve. Keeping pace with spectrum demands will require new technologies that can offer acceptable intercommunication between IoT nodes. In order to address the spectrum scarcity issue, cognitive radio (CR) has drawn drastic attention as a potential technology for future IoT systems [3,4]. The principle of $\mathrm{CR}$ networks (CRNs) lies in identifying additional frequency beings on the same frequency spectrum band with the main spectrum users without disturbing their communication [5]. Those additional CR users are also called secondary users (SUs), while the main spectrum users are known as primary users (PUs).

Generally, wide-band spectrum is divided into non-overlapping channels causing multi-band structure in CRNs. Within CR, spectrum sensing is a key component. In multi-band cooperative spectrum sensing (CSS), instead of sensing all channels, each SU senses multiple channels to determine their occupancy due to hardware limitations and energy consumption constraints. Multi-band CSS based on the CR technology can be a necessary component to fulfill the spectrum demand of future IoT systems owning large numbers of IoT sensor nodes.

Traditionally, CSS can be done in centralized or distributed ways [6]. In the centralized approach, SUs cannot communicate with each other. They forward local sensing information to a central entity, also called fusion center (FC). FC controls the system and makes decisions on the availability of channels based on the feedback from SUs. However, in the distributed approach, SUs exchange their local observations with the corresponding cooperative SUs to determine the availability of channels. Central entity is not needed in this case as the system is decentralized. The nature 
of IoT systems is distributed [3]. In addition to this, IoT networks place focus on learning and feedback information about the environment [4]. Therefore, distributed approach tends to be more favorable in the IoT scenario.

\subsection{Motivation}

In traditional distributed multi-band CSS, SUs sense channels owned by PUs independently, using a single detector type. Then, distributed learning algorithms are used to make cooperative decisions on the availability of channels [6]. However, the nature of IoT comes up with revolutionary ideas. CSS for IoT systems has to consider spectrum analysis, history and prediction, network reconfiguration, and computational

complexity of secondary IoT-nodes [3]. Cognitive IoT systems have to take into account prior information available about signals carried over channels. In addition, scalability is another key IoT-driven requirement, which implies network growth with time [7]. SUs deployed in CRNs may have different types of spectrum detectors due to the heterogeneous nature of IoT systems. Nevertheless, supplementary heterogeneous SUs may cause system performance degradation, leading to extra human intervention in order to get the acceptable performance level back. Therefore, in order to have stable system performance for all channels, it is essential to study and improve distributed multi-band CSS schemes by considering IoT demands mentioned above.

\subsection{Problem Formulation}

In this thesis, we propose a heterogeneous multi-band multi-user CSS (HM2CSS) scheme for distributed IoT systems. Due to the fact that system model is set to be heterogeneous, we consider three types of detectors for local spectrum sensing, which include energy detector (ED), pilot tone-based detector (PD), and orthogonal frequency division multiplexing (OFDM)-based detector (OD). Each SU is equipped with one of the available detectors due to hardware limitations of secondary IoT nodes.

The design of IoT-based multi-band CSS is very challenging mainly because of SUs' scheduling to sense a subset of channels. Correct choice of cooperative SUs may significantly increase system performance level and reduce latency [8]. Furthermore, heterogeneous multi-band multi-user CRNs place serious challenges in terms of 
fairness in the cooperative SU-selection process. Choosing cooperative SUs owning non-similar information about PUs' signal levels is essential for the learning process to be accomplished. In addition, stable system performance as the network size grows is essential in distributed cognitive IoT systems due to the lack of overall centralized control. Hence, a cooperative SU-selection problem is formulated and solved in two stages. In the first stage, SU-leaders, best SUs to listen to for each channel, are formed. An optimization problem of SU-leaders' selection for all channels is formulated depending on the type of the prior information available about the channel. We then analyze the correlation between leaders' and other SUs' received signal envelopes. This information is later on used to formulate another optimization problem in order to decide on the best cooperative SUs for the corresponding channels in the second stage. In our scheme, only SUs with different information owned for the same channel are chosen to be cooperative, which improves the performance of cognitive IoT systems. Consequently, cooperative SUs sense channels and the diffusion learning algorithm is used to determine the occupancy of channels. While implementing the proposed HM2CSS scheme, we solve the challenges of heterogeneity, provide scalability and improvements in terms of detection performance, enhance CRN throughput, and accommodates fair energy consumption on sensing for all channels.

\subsection{Thesis Outline and Contributions}

\subsubsection{Thesis Outline}

The outline of this thesis is as follows. Chapter 2 provides a brief background of IoT as well as CR. It discusses incorporation of the CR technology into IoT systems. Then, the multi-band CSS problem is formulated, and existing CSS schemes are described. We further present several performance measures including detection performance, aggregate CRN throughput, and energy consumption on the sensing process. In Chapter 3, three different network architectures of cognitive IoT systems, named as centralized, distributed, and hybrid, are discussed. The advantages and disadvantages of each one of them are revealed taking into account the nature of IoT. In addition, challenges of multi-band CSS for cognitive IoT systems compared to those for conventional CRNs are explained thoroughly. We consider issues and requirements in designing new multi-band CSS schemes within cognitive IoT systems. Chapter 4 addresses the importance of learning schemes for the distributed approach. 
Our objective is to investigate, which learning schemes are suitable to be used for cognitive IoT systems. Hence, a comparative study between several existing learning approaches is performed, taking into account the challenges of cognitive IoT systems discussed in Chapter 3. Motivated by the requirements of IoT systems, we propose a novel HM2CSS scheme in Chapter 5. Simulation results are provided comparing the proposed HM2CSS scheme to the existing distributed and centralized schemes. Finally, Chapter 6 provides a conclusion with potential future research directions.

\subsubsection{Main Contributions}

The main contributions of this thesis are as follows:

1. A comparative study between three mostly used learning algorithms for distributed CRNs, named as incremental, consensus, and diffusion-based learning, is performed.

(a) Simulation results are provided illustrating the learning process for cases of no link failure and 30\% link failure. System robustness is analyzed in this way.

(b) Simulation results are performed demonstrating the average number of iterations needed to accomplish the learning process for different values of cooperative SUs. These results are further used in order to decide on the scalability abilities of the learning approaches.

(c) Based on this study, we decide on the best suitable learning schemes for cognitive IoT systems. The results illustrate potentials of diffusion and consensus-based algorithms and reveal the disadvantages of the incremental approach for future IoT-based CRNs.

2. We propose a novel multi-band CSS scheme for distributed learning-based cognitive IoT systems named as HM2CSS, which solves the challenges of heterogeneity, provides scalability and improvements in terms of detection performance, enhances CRN throughput, and provides fair sensing energy consumption among SUs and channels.

3. SU-selection is one of the key requirements for CSS. Hence, the proposed HM2CSS scheme first selects leaders among SUs for each channel and then, chooses corresponding cooperative SUs to sense these channels: 
(a) We formulate an optimization problem to choose SU-leaders for all channels. This is done based on the information available about the channels, such that selected SU-leaders are the best SUs to listen to for the corresponding channels.

(b) We then formulate an optimization problem to select corresponding cooperative SUs for each channel, such that SUs with similar information sensed are not selected to be cooperative for the same channel. This is done by means of received signals' correlation analysis. We provide fair distribution of energy consumed on the sensing process for all channels.

(c) We adopt the branch-and-bound (B\&B) algorithm to solve the aforementioned optimization problems. For each one of them, the optimization process is performed concurrently with respect to all channels, independently of the prior information available about them.

4. Simulations are performed to demonstrate the performance of the proposed HM2CSS scheme. The results are compared to the existing multi-band CSS schemes. Simulation results show to improve and provide scalability in terms of global probability of detection, enhance CRN throughput, and provide fair sensing energy consumption among all channels.

\subsection{Notations}

In this thesis, we focus on the discrete-time signal type. Therefore, all signals are formed as column vectors, having the dimensions of $N \times 1$, where $N$ is the number of samples used to sense a signal. Boldface characters are used to express vectors, and boldface upper-case characters are used to express matrices. The corresponding examples are, the received signal $\mathbf{x}=\left[\begin{array}{lll}x_{1} & x_{2} & \ldots \\ x_{N}\end{array}\right]^{T}$, where $(.)^{T}$ indicates the transpose operator, and the identity matrix I. In addition to this, $\mathcal{N}\left(\mu, \sigma^{2}\right)$ and $\mathcal{C N}\left(\mu, \sigma^{2}\right)$ stand for real and complex Gaussian random variables (RVs), respectively, with mean $\mu$ and variance $\sigma^{2}$. All random processes are assumed to be independent and identically distributed (IID). Finally, $Q(x) \triangleq \int_{x}^{\infty} \frac{1}{\sqrt{2 \times \pi}} \times \exp \left(\frac{-t^{2}}{2}\right) d t$ is used as a definition to the standard Gaussian distribution function with $Q^{-1}($.$) as its inverse [9]. Hence, if$ $X \sim \mathcal{N}\left(\mu, \sigma^{2}\right)$, then $\operatorname{Pr}(X \geq x)=Q\left(\frac{x-\mu}{\sigma}\right)$ is the probability that $X \geq x$. 


\section{Chapter 2}

\section{Background}

\section{$2.1 \quad$ Introduction}

In IoT systems, spectrum scarcity is believed to be one of the most crucial problems. In this section, we provide an overview of IoT and CR. Further, we discuss how incorporating $\mathrm{CR}$ into IoT can be one of the solutions to address the spectral efficiency (SE) issue in IoT systems.

\subsubsection{Internet of Things Concept}

Nowadays, IoT can be defined as [10]:

"a loosely coupled, decentralized, and dynamic system in which billions (even trillions) of everyday objects are endowed with intelligence (smartness) to increase their own capabilities and seamlessly communicate and cooperate despite their physical / functional heterogeneities, becoming active participants in business, logistics, information and social processes".

According to [11], the number of devices connected to the Internet is already exceeding the world population as illustrated in Fig. 2.1. It is shown that in 2020, there will be 50 billion devices connected to the Internet. Thus, it is believed that $75 \%$ of network traffic will be accounted for IoT-based devices in 2020 [7]. Most IoT nodes are low-power and are fundamental building blocks of large scale IoT systems. For each IoT node, there should be enough energy and spectrum to transmit information. Moreover, it should be able to communicate reliably and efficiently. However, this places a room for great challenges particularly in the physical layer design of IoT

systems. Not surprisingly, studies claim that in order to support IoT systems, next 


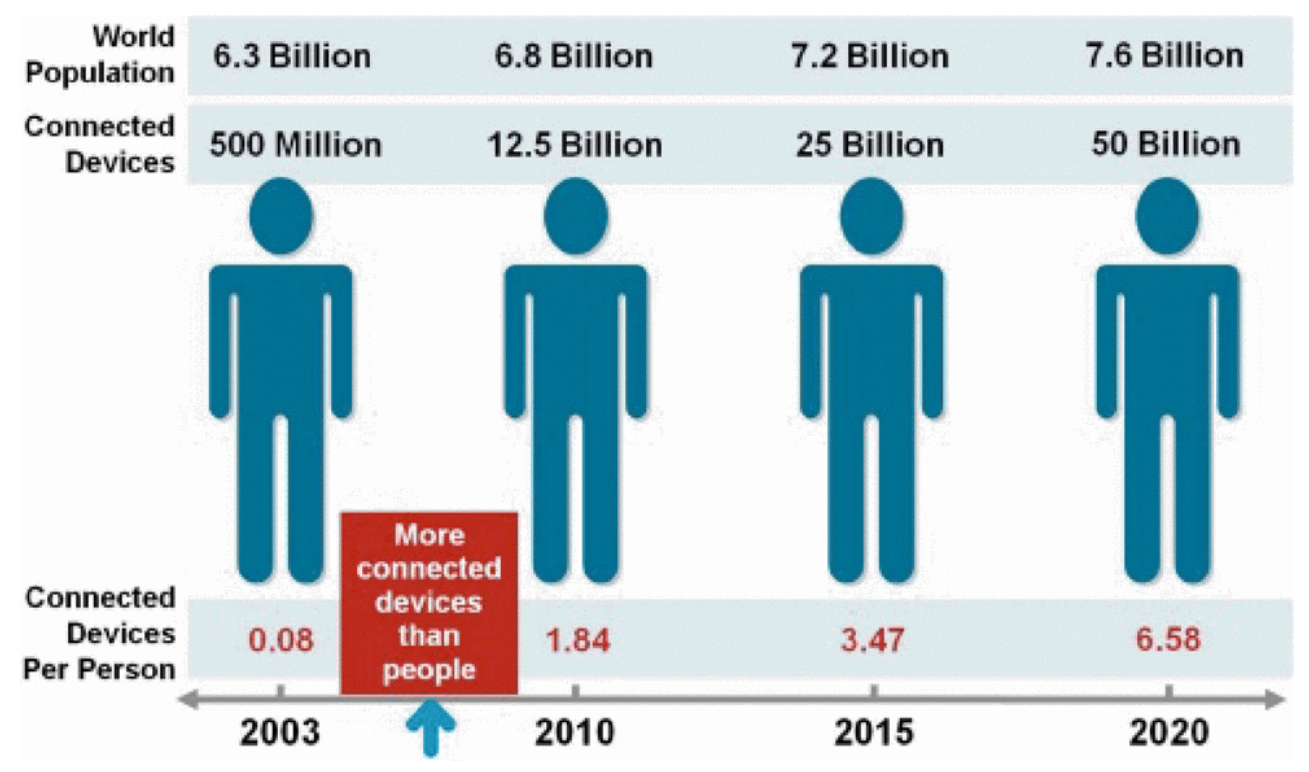

Figure 2.1: Expected growth in the number of devices connected to the Internet [11].

generation networks (NGNs) should have efficient enhancements compared to the current ones: increase in SE 5-15 times, energy efficiency (EE) 100+ times, and cost efficiency (CE) 100+ times [12]. Clearly, IoT challenges should be analyzed from both requirement perspectives, system level and the device one.

The scope of applications deployed by IoT is widely broad ranging from health monitoring and safety to transportation and smart cities. Fig. 2.2 reveals the main domains of IoT applications such as health, agriculture, industry and automation, smart cities, body area networks, security and emergency, connected objects / pets and smart homes, smart grid and metering, as well as intelligent transportation [13].

Concluding, such a wide, heterogeneous, and scalable concept as IoT leads to lots of challenges in terms of physical layer connectivity. Providing communication for an enormous number of heterogeneous devices is one of the hardest tasks. Keeping pace with spectrum and energy demands will require new technologies that can offer acceptable intercommunication between IoT nodes. The following sections will discuss the $\mathrm{CR}$ concept and its potentials to provide solutions for the challenges facing IoT systems mentioned above. 


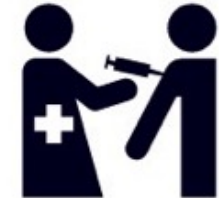

Health

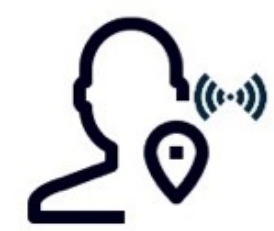

Body area networks

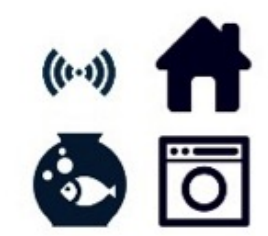

Connected objects, pets, smart homes

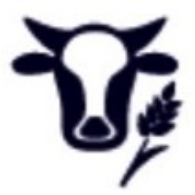

Agriculture

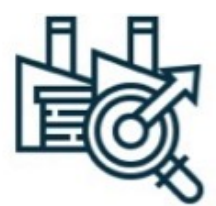

Industry and automation
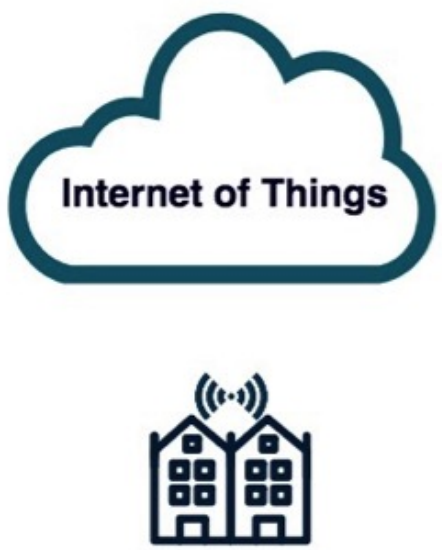

Smart grid and metering

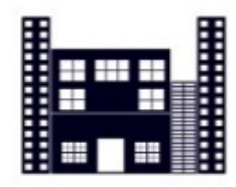

Smart cities
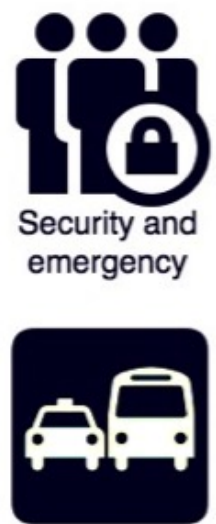

Intelligent transportation

Figure 2.2: Major application domains of IoT.

\subsubsection{Cognitive Radio Overview}

Traditionally, channels are allocated in a static way, which implies the existence of PUs only. Each licensed spectrum band is assigned to a specific licensed service and its PUs. Hence, reallocation of licensed spectrum bands is not allowed. However, this leads to spectrum underutilization [14-17]. In some communication systems, users are most of the time in the idle mode, and frequency channels are left unallocated. An example of such scenario is the case of field spectrum measurements, which took place in New York, showing the maximum aggregate wireless spectrum occupancy of $13.1 \%$ for the bands of $30 \mathrm{MHz}$ to $3 \mathrm{GHz}$ [18]. This reveals the disadvantage of static spectrum allocation. Although static spectrum allocation has its own advantages of a more secure and less interference communication, dynamic approach can help utilize spectrum efficiently solving the spectrum scarcity problem of the future [19].

CR technology appears to be one of the most promising technologies of dynamic spectrum allocation that solves spectrum issues mentioned above. CR roots can be tracked back to 1999, when it was first introduced by J. Mitola [20]. In CRNs, CR 
users / SUs can opportunistically access the spectrum without affecting the transmission of legitimate users / PUs [5]. CR is believed to be a key technology to address spectrum demands in NGNs $[4,21]$. The key of CR lies in self-organizing networks and awareness of the environment. It implies main objectives of highly reliable communication and efficient utilization of radio spectrum by learning from the ambient environment and adapting according to the knowledge gained [22]. The IEEE 802.22 standard for wireless regional area networks is being developed supporting the CR technology to operate over television broadcast bands in an unlicensed way [16].

The CR system architecture consists of three different parts [23]. The first one is the primary network $(\mathrm{PN})$, which represents a set of PUs having the priority to access the spectrum. The secondary part, named as CRN, is a set of SUs, which also need spectrum to communicate, but have less priority to access it. The third part of the system is spectrum itself together with the environment. Conventionally, the CR technology consists of four different modules: spectrum sensing, spectrum decision, mobility, and spectrum sharing, as illustrated in Fig. 2.3. The main cycles of CR are formed as sensing the spectrum, selecting the best frequency bands, coordinating spectrum access with other users, and sharing the spectrum with PUs when allowed

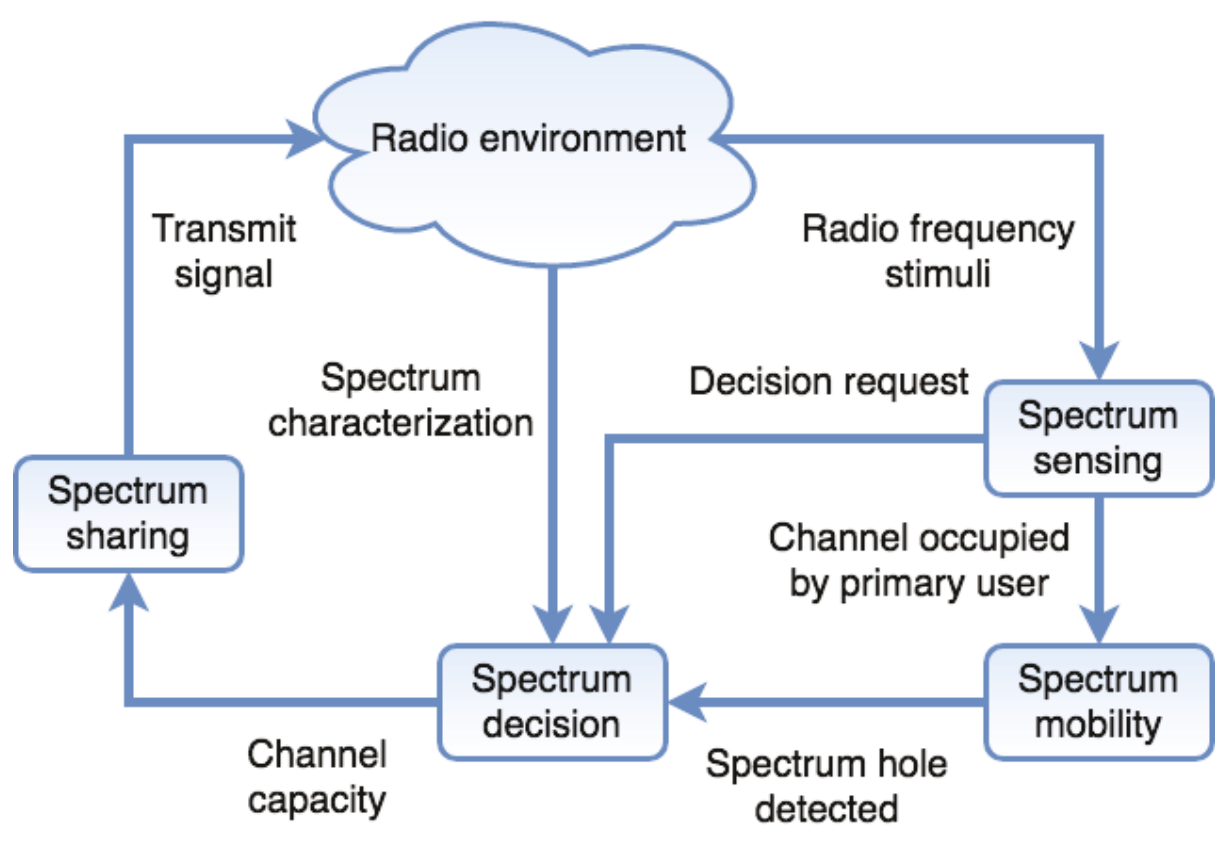

Figure 2.3: The CR cycle overview. 


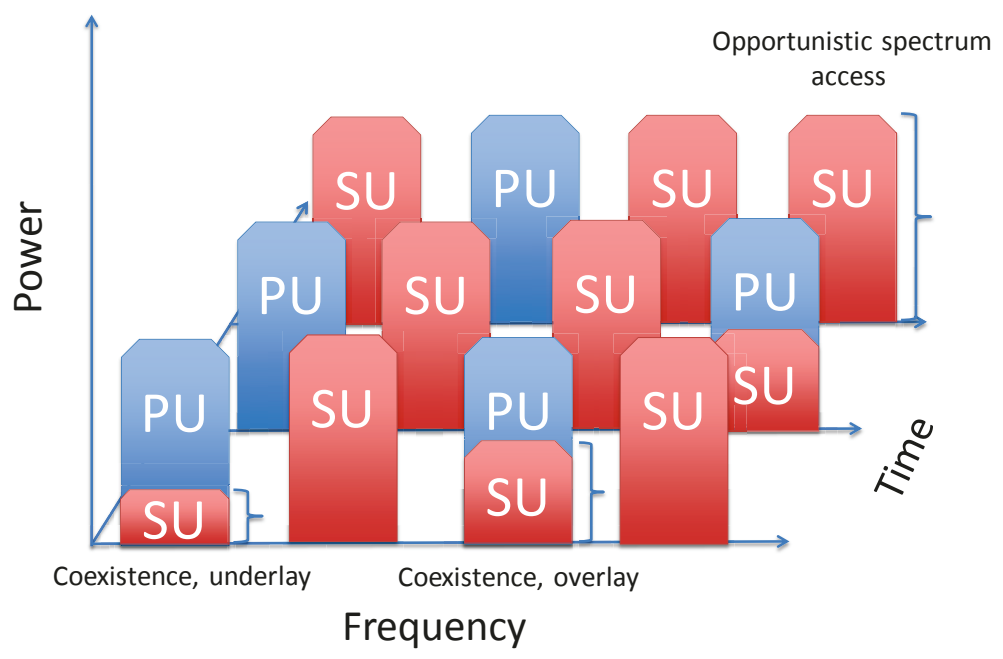

Figure 2.4: The CR paradigms: opportunistic spectrum access, underlay, and overlay.

[24]. The concept of CR lies in allowing SUs to communicate without interrupting PUs by using one of three different paradigms [5]: opportunistic spectrum access, underlay, and overlay. Fig. 2.4 illustrates the three CR paradigms mentioned above. A widely studied form of CR is opportunistic spectrum access [25-27]. It is believed to be the most fundamental CR approach. White spaces are searched in this case. This means that currently unoccupied by PUs bands can be used by SUs. SUs sense channels and decide if PUs are in idle or active modes. In case PUs are not present, SUs are allowed to use the spectrum. However, PUs and SUs cannot coexist on the same channels at once. This means that as soon as PUs appear, SUs have to vacate the corresponding channels. On the other hand, underlay and overlay CR paradigms allow SUs to share spectrum resources concurrently with PUs. The underlay paradigm permits SUs to access the licensed bands of PUs under a certain threshold constraint of the SUs' transmitting power, also called interference temperature [5]. Interference temperature is a metric that evaluates the allowed interference level coming from SUs to be experienced by PUs. In this case, SUs are usually limited to transmitting at low distance ranges due to power level restrictions. As for the overlay paradigm, detailed information of PUs' codebooks and messages is required to be owned by SUs [5]. In this way, SUs are allowed to transmit with higher power levels. Essentially, the 


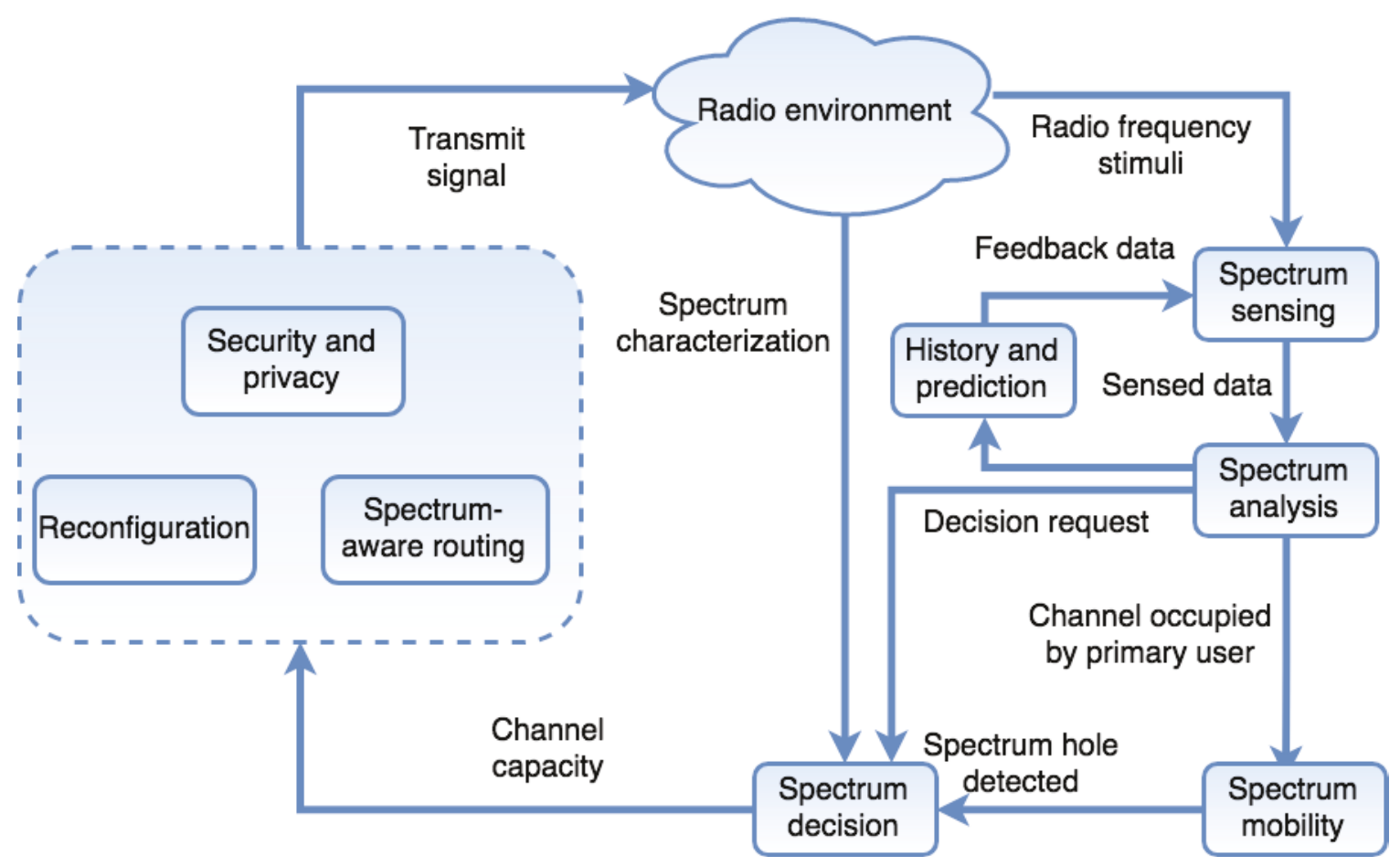

Figure 2.5: IoT-based CR cycle.

communication of PUs and SUs is based upon helping each other in this case. SUs act as relays for PUs, while being allowed to co-share PUs' spectrum bands. One of the major drawbacks of the overlay paradigm is the need of feedback links. In larger networks, feedback may cause overhead. Nevertheless, the technique is still believed to be useful and efficient for smaller networks.

\subsubsection{Incorporating Cognitive Radio into Internet of Things}

Incorporating $\mathrm{CR}$ into IoT brings up additional modules to the $\mathrm{CR}$ cycle, which in total consists of [3]: spectrum sensing, spectrum analysis, history and prediction, spectrum decision, spectrum-aware routing, security and privacy, spectrum-mobility, and reconfiguration, as illustrated in Fig. 2.5. Introducing $\mathrm{CR}$ to IoT can bring the advantages of greater accessibility, better usability, improved adaptability, enhanced interconnectivity, scalability, and enhanced reliability [26]. CR is expected to minimize human intervention in IoT through learning and adaptation.

Multi-band CR technology has drawn drastic attention as a potential solution to 
future IoT networks' spectrum access issues [3,7,27-29]. It is believed to be a necessary component to fulfill the spectrum demand of future IoT systems. Multi-band $\mathrm{CR}$ addresses spectrum underutilization and scarcity problems by allowing SUs to share spectrum together with PUs during their idle mode or concurrently by applying some interference constraints on secondary IoT-nodes. It brings up the advantages of spectrum scarcity relief, cost reduction for spectrum holders, massive connectivity in crowded areas, minimization of human intervention, mobility, monitoring of environment, and learning to IoT systems $[3,7,12]$. Nevertheless, together with advantages, there are several disadvantages in applying it to IoT systems. Among those are interference between PUs-SUs and SUs-SUs, quick out-date of sensed information, requirement of prior information of the PU signal for certain detector types, security, etc. However, those disadvantages can be formed as challenges to be addressed in new multi-band CSS schemes for cognitive IoT systems.

Researchers from academia and industry are investigating CR issues in IoT systems. Various projects devoted to spectrum scarcity and IoT were, are in present, and are approved to be funded in the next few years. A proposal for experimental infrastructure creation, reconfiguration, and extension called "Large-scale Experimentation on Dynamic Spectrum Sharing in IoT" was accepted by European Commission Decision for 2016-2017 years [30]. Furthermore, "5G Long Term Evolution29" facing strategies for spectrum sharing and usage optimization in licensed and unlicensed bands of heterogeneous IoT systems is approved for 2019-2020 years [31]. These examples of projects and research works reveal the importance of the topic for future IoT systems.

The rest of the chapter is structured as follows. In Section 2.2, the multi-band CSS problem is formulated. Then, an overview on existing CSS schemes is given in Section 2.3. Performance measures are presented, which will be used in analyzing the proposed HM2CSS scheme in Section 2.4. Finally, Section 2.5 concludes the chapter with a brief summary. 


\subsection{The Multi-band Cooperative Spectrum Sens- ing Problem}

In non-cooperative spectrum sensing, each $\mathrm{SU}$ senses all channels and then, separately decides if the sensed channels are occupied or not [24]. In contrast with noncooperative spectrum sensing, CSS is using cooperation of multiple SUs by sharing sensed information with each other in order to determine the availability of channels. Cooperation brings the benefit of an increase in reliability of the system. This information is later on used to make decisions on spectrum access by SUs.

Most of the recent research in CSS has focused on the single-band case. However, multi-band CSS can significantly enhance the performance by providing access to a wider range of spectrum, reducing spectrum hand-off, and minimizing energy consumption on spectrum sensing by assigning a subset of channels to each $\mathrm{SU}[6,8]$. Multi-band approach improves system's performance, allowing utilization of carrier aggregation by means of adaptive spectrum support management [32]. Range of low to high communication bandwidths can be implemented, which brings up combining two or more channels to be able to use larger bandwidth for spectrum-hungry applications.

Fig. 2.6 illustrates the multi-band CSS process as a fundamental part of the CR technology. In multi-band CSS, wide-band spectrum can be divided into nonoverlapping channels, also called sub-bands, i.e., $\mathcal{M}=\left\{m_{1}, m_{2}, \ldots m_{M}\right\}$. Each $\mathrm{SU}$ from $\mathcal{K}=\left\{S U_{1}, S U_{2}, \ldots S U_{K}\right\}$ senses multiple channels to determine their occupancy. Due to hardware limitations and energy consumption issues, the maximum amount of channels allowed to be sensed by one SU is $I$, where $I<M$. This means that in practical CRNs, SUs may have different capabilities to sense different channels based on their location and channel state information. Selecting cooperative SUs can help overcome an overhead, especially if several SUs are in deep fade with respect to certain channels or owe similar information after the local sensing process. In addition to this, it helps to reduce the amount of energy consumed on sensing. However, selecting cooperative SUs is a challenging task, which depends on the nature of the network. After cooperative SUs are selected, they have to sense channels assigned to them. Each SU from the cooperative SU-cluster has to solve multiple binary hypothesis problem in the following way: 


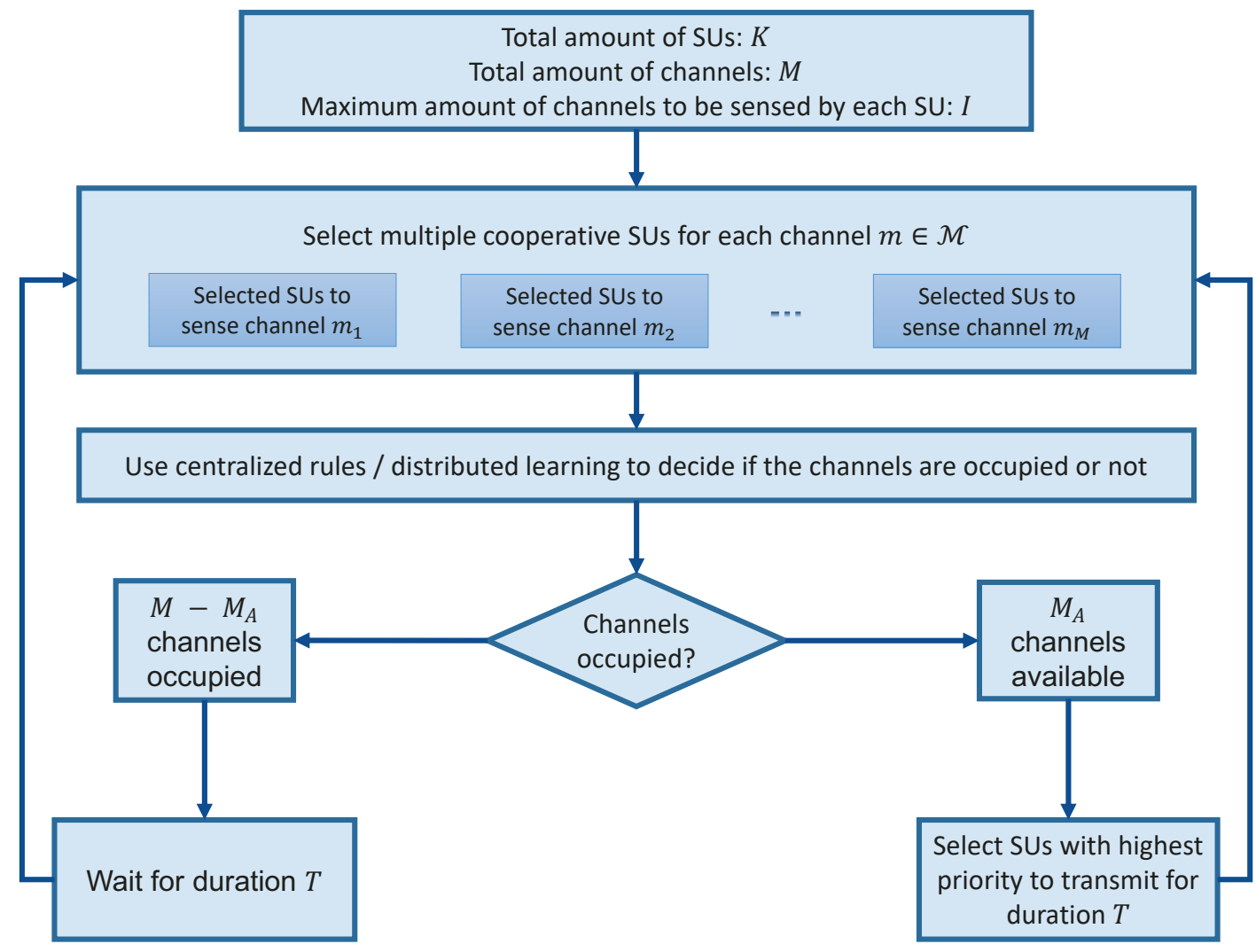

Figure 2.6: A flow chart for multi-band CSS.

$$
\begin{aligned}
& H_{0}^{m, k}: \quad \mathbf{x}_{m}^{k}=\mathbf{w}_{m}^{k} \\
& H_{1}^{m, k}: \quad \mathbf{x}_{m}^{k}=\mathbf{s}_{m}+\mathbf{w}_{m}^{k},
\end{aligned}
$$

where $H_{0}^{m, k}$ and $H_{1}^{m, k}$ denote the absence and presence binary hypothesis testing of the $m$-th channel sensed by $k$-th $\mathrm{SU}$, respectively. $\mathbf{x}_{m}^{k}$ is the signal received by $k$-th SU while sensing $m$-th channel, $\mathbf{s}_{m}$ is the signal transmitted by a PU over the $m$-th channel, and $\mathbf{w}_{m}^{k}$ is the circularly symmetric complex Gaussian noise with zero mean and $\left(\sigma_{m}^{k}\right)^{2}$ variance, i.e., $\mathbf{w}_{m}^{k} \sim \mathcal{C N}\left(\mathbf{0},\left(\sigma_{m}^{k}\right)^{2} \mathbf{I}\right)$. The solution of the demonstrated hypothesis problem has the form of [6]:

$$
\Lambda\left(\mathbf{x}_{m}^{k}\right) \underset{H_{0}^{m, k}}{\stackrel{H_{1}^{m, k}}{\lessgtr}} \zeta_{m}^{k},
$$

where $\Lambda($.$) is the test statistics and \zeta_{m}^{k}$ is the corresponding threshold, which depends on the detector type of the corresponding SU and the information available about the 
PU signal as well as the channel. In case test statistics is greater than the threshold, the PU is believed to be in the active mode. Nevertheless, if test statistics is less than the corresponding threshold, the $\mathrm{PU}$ is believed to be in the idle mode and a $\mathrm{SU}$ is allowed to access the channel. Later, depending on the network structure of either centralized or distributed types, certain rules or learning algorithms are being used, respectively, to cooperatively decide on the occupancy of channels.

\subsection{Related Work}

There have been a plethora of research efforts in recent years in the area of CSS. This section gives a brief state-of-the-art summary of this field.

To begin with, authors propose an energy-efficient reliable decision transmission scheme for IoT-based CSS in [33]. The proposed scheme has a centralized system model which uses the OR / AND-rule and improves the probability of detection as well as reduces energy consumption on the sensing process. However, the aforementioned work does not take into account the multi-band approach. Recently, several multiband spectrum sensing schemes are proposed in literature. The non-uniform sensing duration of multi-band spectrum access is analyzed for centralized CRNs in [15]. Authors in [34] propose an efficient ED-based centralized multi-band CSS scheme, which uses the detection threshold optimization to minimize energy consumption on the sensing process. A basic centralized multi-band spectrum sensing scheme is proposed in [8] to reduce the complexity of the spectrum sensing process. Authors provide a thorough throughput analysis of the proposed system. Nevertheless, all works mentioned above do not take into account either the heterogeneous aspect of SUs or the distributed system topology.

In [35], an optimal multi-band distributed homogeneous CSS scheme is proposed, which maximizes the CRN throughput with constraints on energy and signal processing resources consumed on sensing. Moreover, in [36], authors propose a homogeneous distributed persistent spectrum sensing strategy (PSSS), which uses PU traffic patterns to determine channels to be sensed for multi-band spectrum sensing of centralized CRNs. Further, authors propose an adaptive spectrum sensing strategy, which uses PSSS or random spectrum sensing strategy (RSSS) to select channels to be sensed. In [37], a heterogeneous centralized system using k-out-of-K rule is analyzed. Centralized and distributed consensus learning-based heterogeneous CRNs are 
compared in terms of CRN throughput analysis in [6]. There, cooperative SUs that sense a certain number of channels are chosen arbitrarily, i.e., in a random manner. Nevertheless, random SU-selection may lead to system performance degradation as the network size increases.

There exists plenty solutions for cooperative SU-selection schemes for single-band CRNs. In [38], a single channel CRN is assumed, where one user is chosen randomly and then, Cramer-Von Mises (CVM) test values are calculated to select the rest cooperative SUs. Only those SUs are selected to be cooperative, for which CVM test values are greater than the calculated threshold. In [39], a distributed heuristic algorithm is proposed to reduce the energy consumption of the system by minimizing the number of cooperative SUs selected. However, in this system, SUs located within a certain distance to a PU are not allowed to use the spectrum and act only as sensing units. As it can be seen, the works mentioned above do not consider SU-selection in the heterogeneous multi-band context.

Concluding, non-arbitrary ways of assigning channels to be sensed by participating SUs in heterogeneous multi-band multi-user distributed CRNs are not considered in the existing literature. Hence, we propose a multi-band CSS scheme, named as HM2CSS, for distributed CRNs to accommodate heterogeneity and improve detection performance and CRN throughput of IoT systems.

\subsection{Performance Measures}

In this section, a brief overview is given to the performance metrics that will be employed to test and evaluate the proposed HM2CSS scheme.

\subsubsection{Detection Performance}

Traditionally, spectrum sensing performance is evaluated by analyzing two probabilities as follows: 1) the probability of detection, which represents the probability that a SU detects a PU correctly, while the PU is in the active mode and 2) the probability of false alarm, which reveals incorrect decision of a SU that a PU is present while the $\mathrm{PU}$ is in the idle mode. Mathematically, the probability of detection, also called PU protection level, is expressed as follows:

$$
P_{d}\left(\zeta_{m, k}\right) \triangleq \operatorname{Pr}\left(\Lambda\left(\mathbf{x}_{m}^{k}\right)>\zeta_{m, k} \mid H_{1}^{m, k}\right)=1-F_{\Lambda \mid H_{1}^{m, k}}\left(\zeta_{m, k}\right)
$$


whereas the probability of false alarm can be expressed as follows:

$$
P_{f}\left(\zeta_{m, k}\right) \triangleq \operatorname{Pr}\left(\Lambda\left(\mathbf{x}_{m}^{k}\right)>\zeta_{m, k} \mid H_{0}^{m, k}\right)=1-F_{\Lambda \mid H_{0}^{m, k}}\left(\zeta_{m, k}\right),
$$

where $F_{\Lambda \mid H_{1}^{m, k}}($.$) and F_{\Lambda \mid H_{0}^{m, k}}($.$) are the cumulative distribution functions (CDFs) of$ $\Lambda\left(\mathbf{x}_{m}^{k}\right)$ under $H_{1}^{m, k}$ and $H_{0}^{m, k}$, respectively.

The two parameters mentioned above introduce two degrees of freedom to spectrum sensing problems. In particular, the higher the $P_{d}$ value is the more the PN is protected from the interference caused by the CRN. This interference occurs, when a PU is incorrectly detected to be absent, hence both PU and SU use the channel. Furthermore, the lower the $P_{f}$ values are, the higher the CRN throughput can be achieved. This can be explained by the fact that when a PU is not using the wireless spectrum and a $\mathrm{SU}$ incorrectly detects that the PU is present, the spectrum is not being utilized. This trade-off leads to the rationale behind the Neyman-Pearson framework, where the objective is to maximize the value of $P_{d}$ subject to a predetermined value of $P_{f}[40]$ :

$$
P_{d}=1-F_{\Lambda \mid H_{1}^{m, k}}\left(F_{\Lambda \mid H_{0}^{m, k}}^{-1}\left(1-P_{f}\right)\right),
$$

where $F_{\Lambda \mid H_{0}^{m, k}}^{-1}($.$) is the inverse function of F_{\Lambda \mid H_{0}^{m, k}}($.$) .$

Different detector types imply different detection probabilities for the corresponding probabilities of false alarms. As mentioned before, we consider either ED, PD or OD-based scenarios for local spectrum sensing by each SU. In order to determine the availability of a channel in the ED-based case, the received signal power is estimated, whereas for the PD-based scenario, the orthogonality property between the pilot tone and the data-carrying signal is used $[6,41]$. As for the OD-based case, using the knowledge of cyclic prefix and the OFDM symbol length, the inherent signal correlation incurred by the cyclic prefix repetition is taken into consideration [42]. This is why different PU signal structures in three different cases lead to different PU protection levels of the corresponding probabilities of false alarm.

\subsubsection{Energy Detection}

When a SU does not have any prior information about the PU signal across the $m$-th channel, it is usually modeled as a random Gaussian signal with zero mean and $\left(\sigma_{s}^{m}\right)^{2}$ variance, i.e., $\mathbf{s}_{m} \sim \mathcal{N}\left(\mathbf{0}\right.$, i.e. $\left.\left(\sigma_{s}^{m}\right)^{2} \mathbf{I}\right)$. Mathematically, the probability of detection for 
the $k$-th ED-based SU sensing $m$-th channel is expressed as follows [6]:

$$
P_{d, m k}^{E}=Q\left(\frac{Q^{-1}\left(P_{f}\right)-\sqrt{N_{m}^{k}} \times S N R_{m k}}{\sqrt{S N R_{m k}+1}}\right),
$$

where $N_{m}^{k}$ is the corresponding number of samples for the $k$-th $\mathrm{SU}$ to sense the $m$-th channel, $S N R_{m k}$ is the signal-to-noise ratio (SNR) value of the $k$-th $\mathrm{SU}$ for the $m$-th channel, and $Q($.$) as well as Q^{-1}($.$) are the standard Gaussian distribution function$ and its inverse, respectively.

Fig. 2.7 illustrates a block diagram of the ED-based detection case. Due to the fact that ED-based detection is done by estimating the received energy level of the PU signal, regardless the noise level is high or low, together with the PU signal it is detected. In case noise level is high and the PU is in the idle mode, a SU may decide that the PU is present. This is why the SNR value of the channel plays an important role in this type of detection. The test statistics for the ED-based detection can be expressed as follows:

$$
\Lambda^{E}\left(\mathbf{x}_{m}^{k}\right)=\frac{1}{N_{m}^{k}} \times\left\|\mathbf{x}_{m}^{k}\right\|^{2},
$$

and the corresponding threshold for the ED-based detection is denoted as [43]:

$$
\zeta_{m, k}^{E}=\frac{1}{N_{m}^{k}} \times Q^{-1}\left(P_{f}\right)+1 .
$$

\subsubsection{Pilot Tone Detection}

When a SU has the information about the pilot tone of the PU signal sent across the $m$-th channel, then PD-based detection can be used in order to determine the

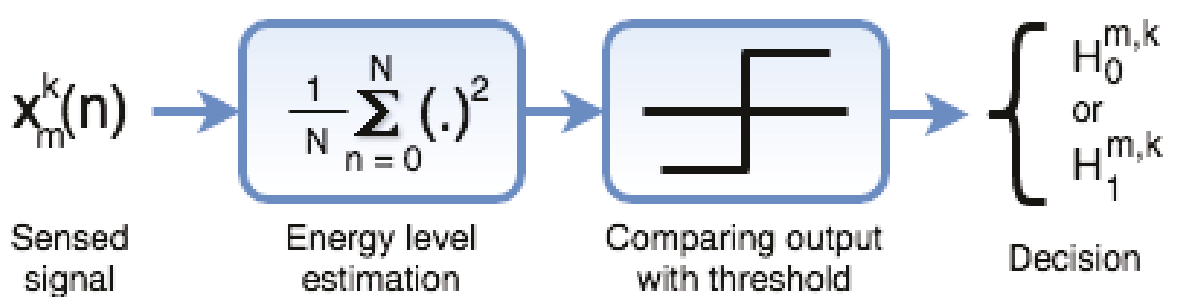

Figure 2.7: Block diagram of the ED-based case. 
occupancy of the m-th channel. Fig. 2.8 illustrates a block diagram of the PDbased scenario. In this case, the PU data-carrying signal part is still modeled as a random Gaussian signal with zero mean and $\left(\sigma_{s_{d}}^{m}\right)^{2}$ variance, i.e., $\mathbf{s}_{m, d} \sim \mathcal{N}\left(\mathbf{0},\left(\sigma_{s_{d}}^{m}\right)^{2} \mathbf{I}\right)$. However, the pilot tone of this signal, $\mathbf{s}_{m, p}$, is known and is believed to be orthogonal to the data-carrying part. Mathematically, a PU signal can be expressed as:

$$
\mathbf{s}_{m}=\sqrt{\theta} \times \mathbf{s}_{m, p}+\sqrt{1-\theta} \times \mathbf{s}_{m, d},
$$

where $\mathbf{s}_{m, d}$ is the data-carrying part, $\mathbf{s}_{m, p}$ is the pilot tone part of the $m$-th PU signal, and $\theta$ is the power allocation fraction to the pilot tone. The probability of detection for the $k$-th PD-based SU sensing $m$-th channel is expressed as follows [41]:

$$
P_{d, m k}^{P}=Q\left(Q^{-1}\left(P_{f}\right)-\sqrt{2 \times \theta \times N_{m}^{k} \times S N R_{m k}}\right) .
$$

Due to the fact that the data-carrying part of the PU signal is orthogonal to the known pilot tone signal, the detection is done by estimating the pilot tone signal, ignoring the presence of the data-carrying part. The test statistics for the PD-based detection can be written as follows:

$$
\Lambda^{P}\left(\mathbf{x}_{m}^{k}\right)=\frac{1}{N_{m}^{k}} \times \sum_{n=1}^{N_{m}^{k}} x_{m}^{k}(n) \times s_{m, p}(n) .
$$

The corresponding threshold for the PD-based detection is expressed as:

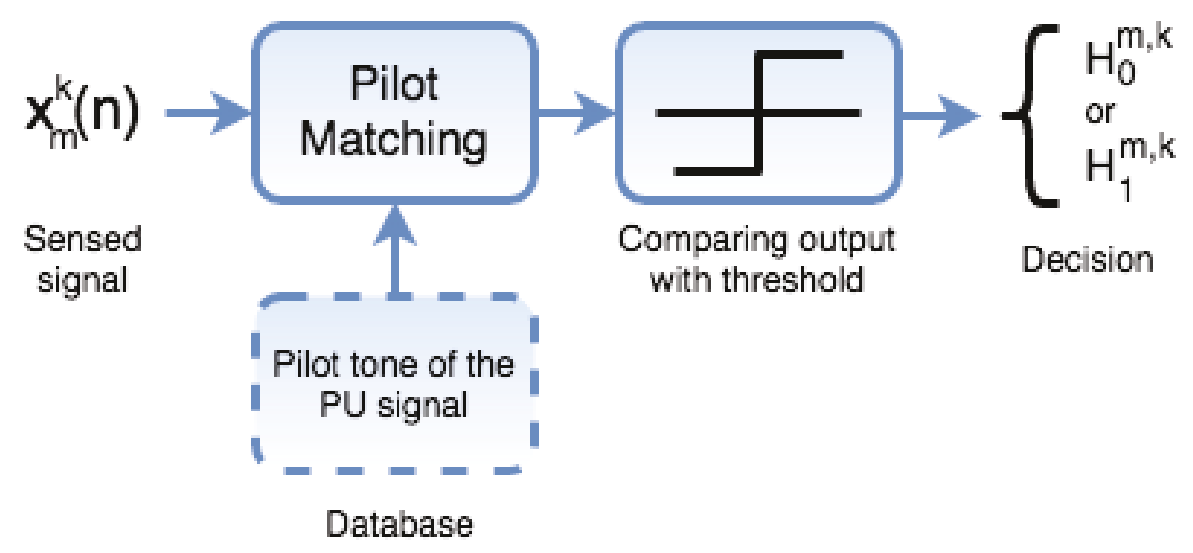

Figure 2.8: Block diagram of the PD-based case. 


$$
\begin{aligned}
\left.\left.\zeta_{m, k}^{P}=\sqrt{P \times \frac{\left(\sigma_{m}^{k}\right)^{2}}{N_{m}^{k}} \times Q^{-1}\left(1-Q\left(\sqrt{2 \times N_{m}^{k} \times \theta \times S N R_{m k}}\right.\right.}-Q^{-1}\left(P_{f}\right)\right)\right) \\
+P \times \sqrt{\theta},
\end{aligned}
$$

where $P$ is the total power allocated for the PU signal consisting of the data-carrying part and the pilot tone and $\left(\sigma_{m}^{k}\right)^{2}$ is the corresponding noise power.

\subsubsection{OFDM-based Detection}

When the PU signal is known to be OFDM, the OD-based scenario can be used. Fig. 2.9 illustrates a block diagram of the OD-based case. Using the knowledge of cyclic prefix and the OFDM symbol length, the inherent signal correlation incurred by cyclic prefix repetition is utilized to detect the PU signal. Mathematically, the probability of detection for the $k$-th OD-based SU sensing $m$-th channel is expressed as follows [42]:

$$
P_{d, m k}^{O} \approx Q\left(\frac{Q^{-1}\left(P_{f}\right)-\sqrt{2 \times N_{m}^{k}} \times S N R_{m k}}{\sqrt{4 \times S N R_{m k}+1}}\right) .
$$

Generally, an OFDM signal is assumed to be the summation of narrow-band phase shift keying (PSK) or quadrature amplitude modulated (QAM) sub-carriers. An OFDM signal may consist of several blocks, each one having a cyclic prefix added

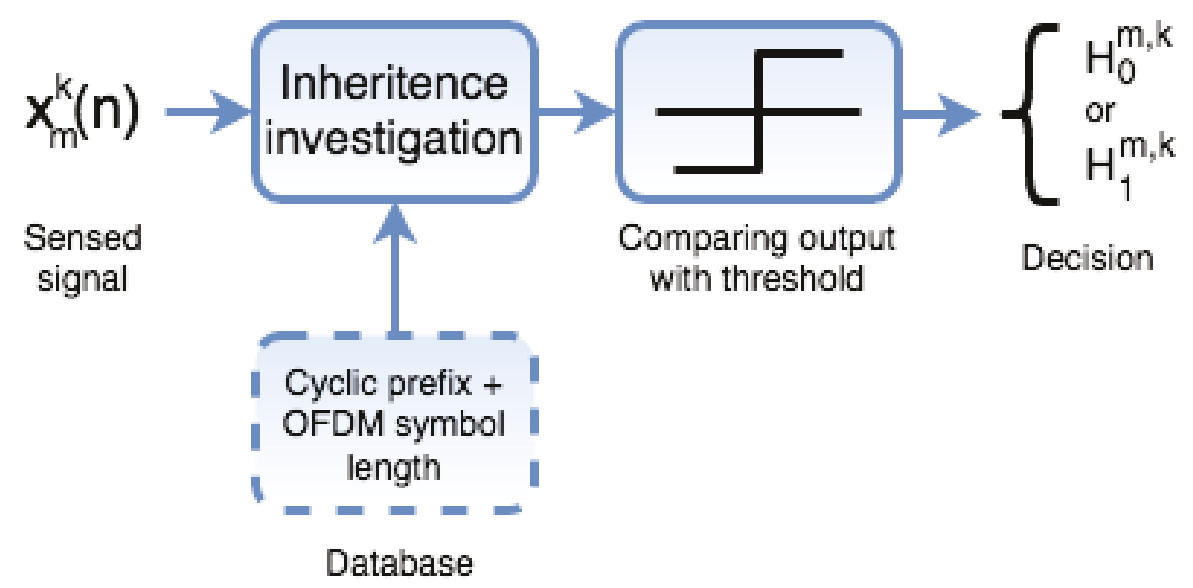

Figure 2.9: Block diagram of the OD-based case. 
to the front. Mathematically, the test statistics for the OD-based scenario can be specified as [44]:

$$
\Lambda^{O}\left(\mathbf{z}_{1}^{m k}, \mathbf{z}_{2}^{m k}\right)=2 \times \frac{\sum_{n=1}^{2 \times N_{m}^{k}} z_{1}^{m k}(n) \times z_{2}^{m k}(n)}{\left\|\mathbf{z}_{1}^{m k}\right\|^{2}+\left\|\mathbf{z}_{2}^{m k}\right\|^{2}} .
$$

where $\mathbf{z}_{1}^{m k}$ and $\mathbf{z}_{2}^{m k}$ are expressed as follows:

$$
\begin{aligned}
\mathbf{z}_{1}^{m k}= & {\left[x_{m, r}^{k}(1) x_{m, i}^{k}(1) x_{m, r}^{k}(2) x_{m, i}^{k}(2) \ldots x_{m, r}^{k}\left(N_{m}^{k}\right) x_{m, i}^{k}\left(N_{m}^{k}\right)\right], } \\
\mathbf{z}_{2}^{m k}= & {\left[x_{m, r}^{k}\left(T_{d}+1\right) x_{m, i}^{k}\left(T_{d}+1\right) x_{m, r}^{k}\left(T_{d}+2\right) x_{m, i}^{k}\left(T_{d}+2\right) \ldots\right.} \\
& \left.x_{m, r}^{k}\left(T_{d}+N_{m}^{k}\right) x_{m, i}^{k}\left(T_{d}+N_{m}^{k}\right)\right]
\end{aligned}
$$

with $T_{d}$ being the number of symbols in one OFDM block and $\mathbf{x}_{m, r}^{k}$ as well as $\mathbf{x}_{m, i}^{k}$ denoting the real and imaginary vectors of $\mathbf{x}_{m}^{k}$, respectively. The corresponding threshold for the OD-based case can be expressed as follows:

$$
\zeta_{m, k}^{O}=\frac{1}{N_{m}^{k}} \times Q^{-1}\left(2 \times P_{f}\right) .
$$

Fig. 2.10 illustrates an analytical overview of the detection performance for the three different methods discussed above in a single SU single-band scenario under the additive white Gaussian noise (AWGN) channel conditions. The number of samples sensed and the probability of false alarm for the ED, PD, and OD-based scenarios are set to be $N=2000$ and $P_{f}=10 \%$, respectively. It is clear that the more information a $\mathrm{SU}$ owns about the PU signal, the better the detection performance is.

\subsubsection{Cognitive Radio Network Throughput}

The effectiveness of CSS is mainly measured by the achievable CRN throughput. Traditionally, in CRNs a SU can access a channel whether the PU is in the idle or active mode by means of opportunistic or underlay / overlay CR paradigms, respectively [5]. In case of underlay / overlay, it is important to analyze the aggregate throughput of both PN and CRN. However, in our analysis the opportunistic paradigm is used, where spectrum is being accessed by a SU only when the PU is in the idle mode. This is why the PN throughput analysis can be omitted as the CRN does not provide interference to the PN. Yet, the CRN throughput analysis stays of great importance. 


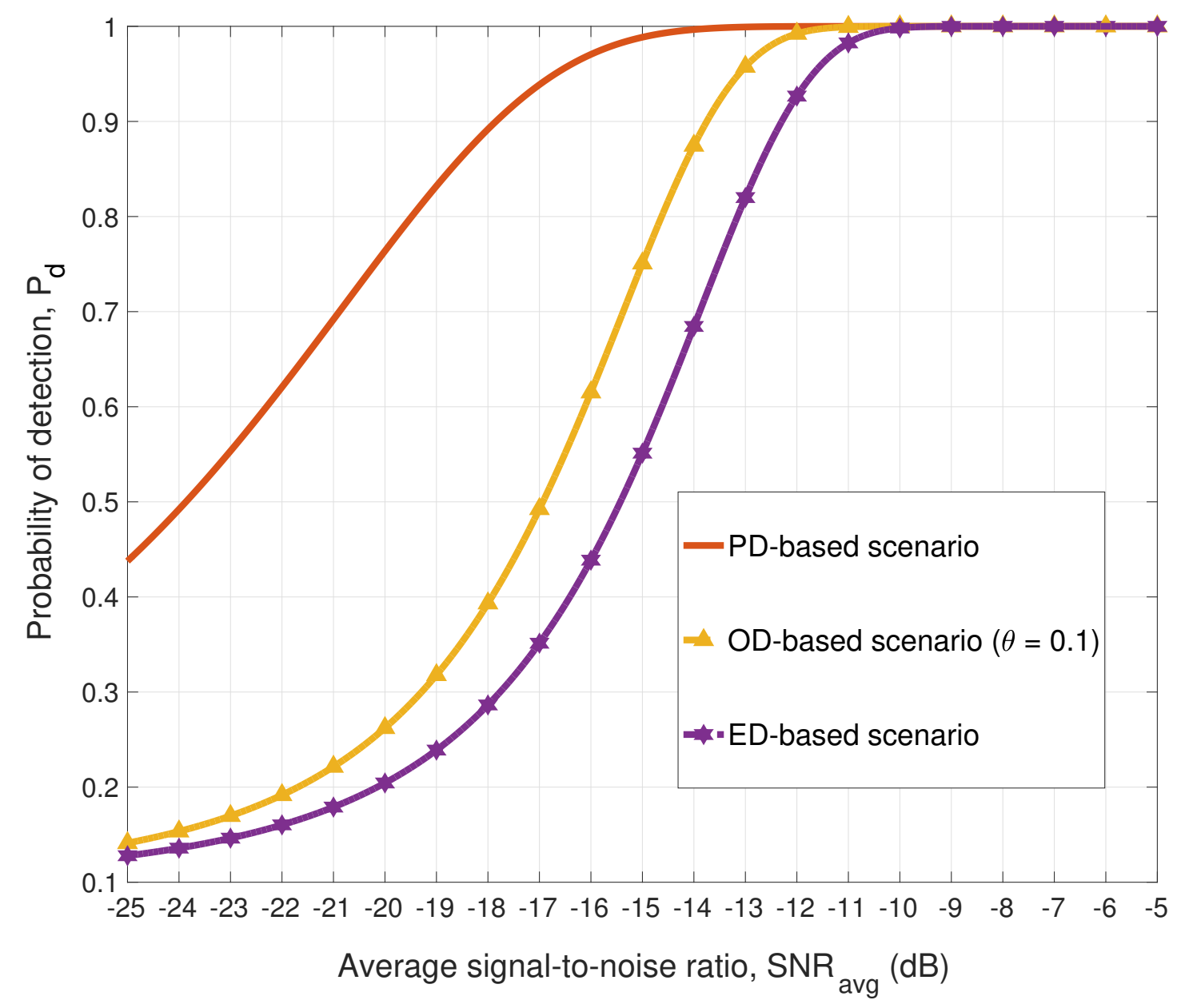

Figure 2.10: Detection performance overview for PD, OD, and ED-based scenarios.

Assuming SUs access $L$ channels out of $M$, the aggregate CRN throughput can be defined as:

$$
\begin{array}{r}
R=B \times \sum_{m=1}^{L} p_{0}^{m} \times \log _{2}\left(1+S N R_{m k}\right) \times\left(1-P_{f, m k}\right) \\
+p_{1}^{m} \times \log _{2}\left(1+S I N R_{m k}\right) \times\left(1-P_{d, m k}\right),
\end{array}
$$

where $B$ is the corresponding channel bandwidth, $p_{0}^{m}$ denotes the probability that the $m$-th channel is in the idle mode, $p_{1}^{m}$ denotes the probability that the $m$-th channel is in the active mode, $S N R_{m k}$ is the SNR value of $k$-th SU for the $m$-th channel, and $S I N R_{m k}$ is the signal-to-interference-and-noise ratio (SINR) value of $k$-th SU for the $m$-th channel, when the interference is coming from mis-detected PUs being in an 
active mode.

\subsubsection{Energy Consumption on the Sensing Process}

Another important parameter for CRNs is energy consumption on the sensing process. Fig. 2.11 illustrates a single frame structure of the CSS process. In general, let $T_{s}^{m}$ denote the total time for a $\mathrm{SU}$ to sense a channel, where $T_{s}^{m}$ is proportional to the number of samples being used to sense the $m$-th channel and $T$ be the transmission or idle mode time. Then, $T_{F}$ is the total period of the frame such that:

$$
T_{F}=T_{S}^{m}+T .
$$

The energy consumed to sense each channel is represented by $E_{m, C O N S}$ and can be given as:

$$
E_{m, C O N S}=\left(P_{S} \times T_{S}^{m}\right) \times Q_{m},
$$

where $P_{S}$ is the power consumed on the sensing process and $Q_{m}$ is the number of cooperative SUs sensing the $m$-th channel. Therefore, aggregate CRN energy consumption on the sensing process can be expressed as:

$$
E_{T, C O N S}=\sum_{m=1}^{M}\left(P_{S} \times T_{S}^{m}\right) \times Q_{m} .
$$

Sensing time Transmission / idle mode time

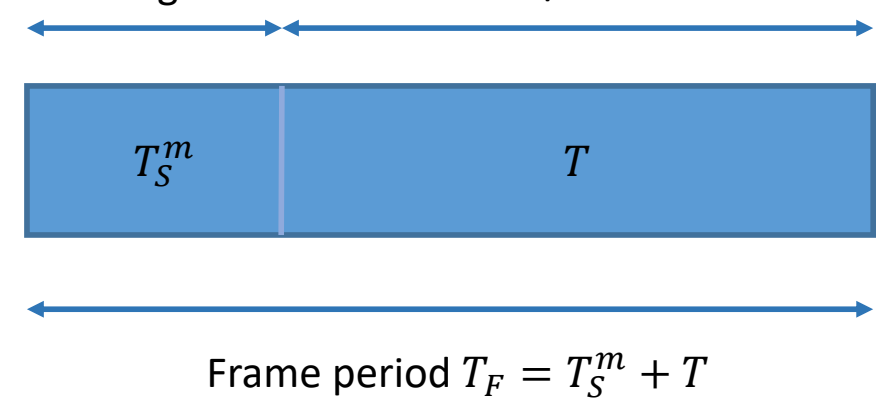

Figure 2.11: The structure of one frame for CSS. 


\subsection{Summary}

In this chapter, a short introduction to IoT and CR concepts was given. The importance of $\mathrm{CR}$ for IoT systems was justified by the spectrum scarcity issue coming from a large number of IoT devices. Then, the multi-band CSS problem was discussed as a part of supporting billions of IoT devices' pervasive connectivity. In addition to this, a summary on the existing IoT-based CSS schemes was given. Finally, performance measures to be used in the thesis in order to evaluate the proposed HM2CSS scheme were presented, including the detection performance, CRN throughput, and energy consumption on the sensing process. 


\section{Chapter 3}

\section{Multi-band Cooperative Spectrum Sensing in Cognitive IoT Systems: Architectures and Challenges}

\subsection{Introduction}

Providing spectrum access to billions of IoT devices is one of the paramount issues for IoT systems. CR is believed to be a prominent solution to spectrum scarcity caused by IoT applications, where multi-band CSS is a key component. Nevertheless, cognitive IoT systems expose a number of challenges yet to overcome in the design of multi-band CSS. Conventional multi-band CSS techniques can be improved in order to meet the challenges of IoT systems.

The outline of this chapter is as follows. Section 3.2 introduces the potential cognitive IoT system architecture types. Sections 3.2.1 and 3.2.2 discuss centralized and distributed cognitive IoT system architectures, respectively, whereas the potential strength of the hybrid topology combination is revealed in Section 3.2.3. In addition to this, we provide a pervasive list of challenges associated with the design of multiband CSS schemes for cognitive IoT systems in Section 3.3. Finally, Section 3.4 concludes the chapter with a brief summary.

\subsection{IoT-based System Architecture}

Traditionally, CSS for cognitive IoT systems can be done in either centralized or distributed ways. This leads to the existence of three main architecture structures: 
centralized, distributed, and the hybrid combination of centralized and distributed approaches. Cognitive IoT systems place a great challenge in terms of network architecture due to the variety of applications supported and their needs. All these network architectures offer their own advantages and disadvantages. For instance, centralization shows better performance and security, whereas distributed systems allow system adaptation and no single point failure. However, it is up to the IoT application to determine which topology works best in specific scenarios.

\subsubsection{Centralized Cognitive IoT Network Architecture}

In centralized networks, a central entity, also called FC, collects the sensing data from secondary IoT-nodes and based on it, makes decisions on the availability of spectrum channels using OR / k-out-of-K / majority / AND rules [8]. An example of such system can be seen in Fig. 3.1(a). Here, a heterogeneous secondary IoT network is presented. This type of networks provides great system performance due to high system control level. However, because of the fact that information exchange is performed only with an FC only, in case a link is in deep fade the performance may degrade drastically [6]. Moreover, problems occur when a FC fails as the network stops working. Finally, one of the main issues with centralized CRNs is latency. In addition to sensor processing and network transmission, FC processing is another source of latency for centralized CRNs. For instance, when a typical white space lasts for dozens of milliseconds, yet due to the FC processing its detection takes hundreds of milliseconds. This means that the exploitation of these white spaces is unjustified [45].

\subsubsection{Distributed Cognitive IoT Network Architecture}

In distributed networks, secondary IoT-nodes cooperate by exchanging locally sensed information between each other and using learning algorithms come up with the common decisions on the availability of channels. The system is completely decentralized, which means it lacks the overall control. An example of such a system is illustrated in Fig. 3.1(b). Despite lower performance than the centralized one, distributed networks are easily adapted in real time and are robust against topology changes. This presents a huge advantage to mobile and scalable IoT systems. In addition to this, locality represents great importance in IoT systems [46]. In contrast to the centralized 

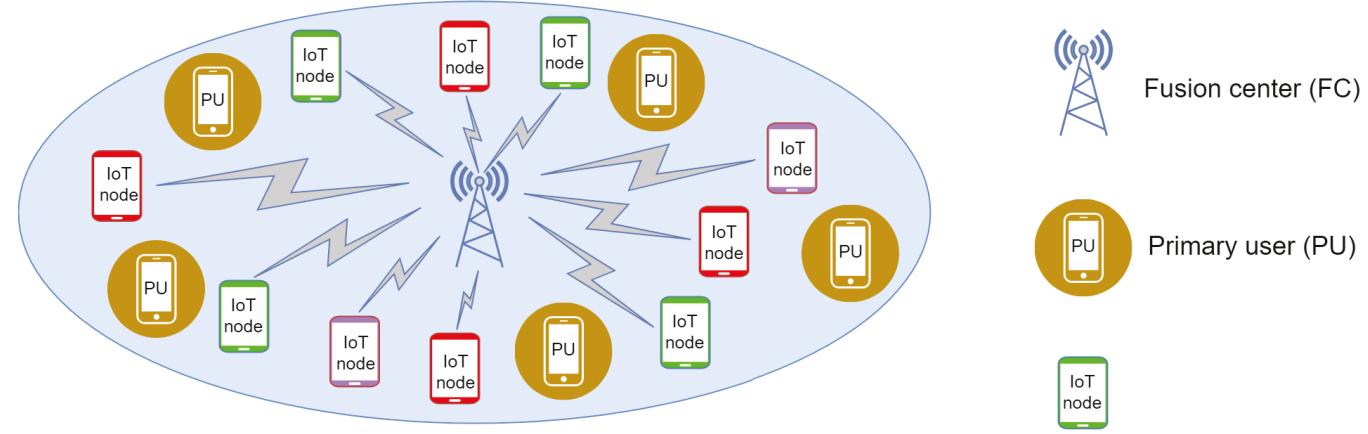

(a)
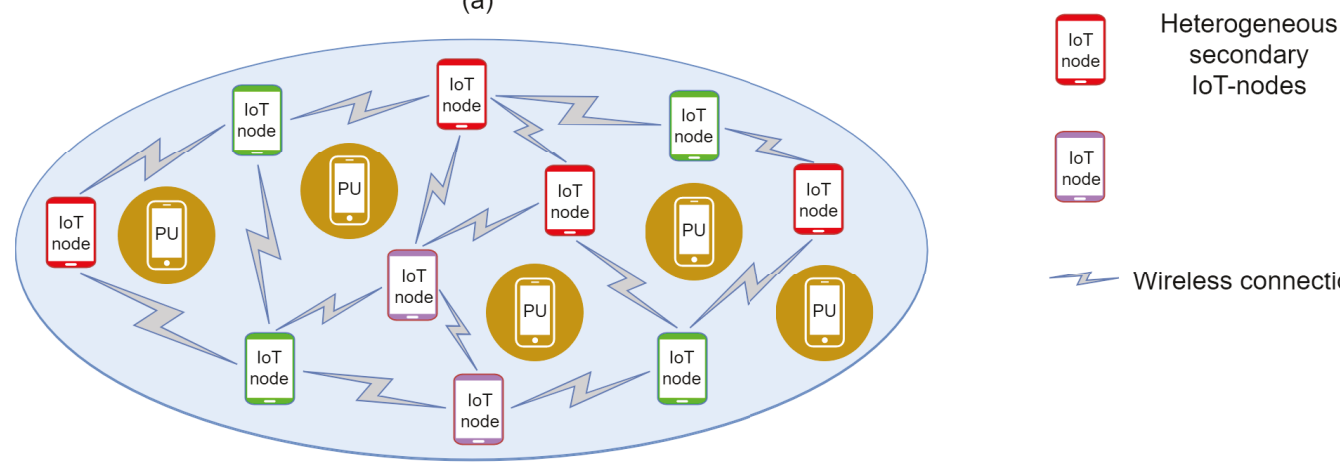

(b)

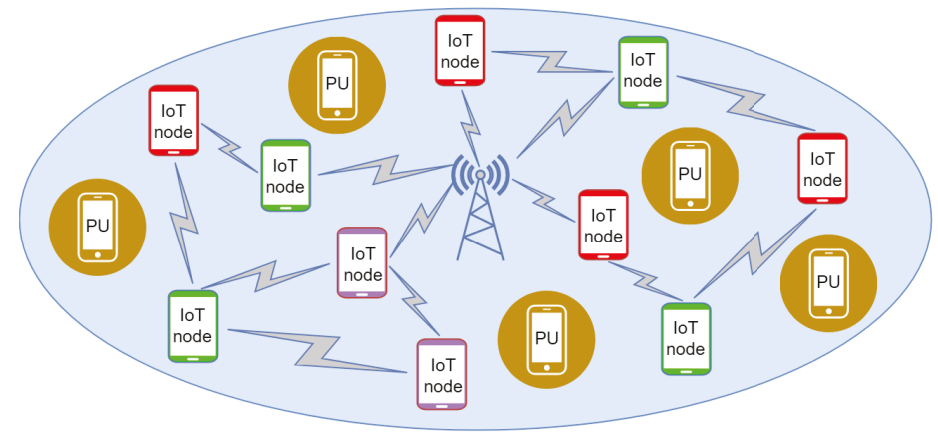

(c)

Figure 3.1: Heterogeneous secondary IoT-based networks: (a) centralized, (b) distributed, and (c) hybrid.

case, distributed network architecture allows network analysis not only on the global / wide level but local as well. It is proved that user experience improvement can be achieved while integrating local and wide area as a new form of cooperation in IoT systems [12,21]. This is why cognitive IoT systems are mainly focused on distributed network architectures. 


\subsubsection{Hybrid Cognitive IoT Network Architecture}

IoT systems represent a summation of networks, which can be centralized and distributed. Hence, this leads to the existence of hybrid system topology. In fact, hybrid systems may be sufficiently used in analyzing the borders of centralized and distributed networks. To begin with, self organizing networks is one of the forms of hybrid topologies [10]. Hybrid systems support spectrum management coordination based on adjustment of both centralized and distributed cases and can utilize a dynamic architecture choice depending on the application. Another approach of the hybrid topology is the umbrella one [47]. Depending on the application, centralized or distributed femto / pico-cell IoT-based CRNs can coexist under a PU macro-cell. A femto / pico-cell clustering scheme is believed to manage wireless resources of the heterogeneous IoT systems. Fig. 3.1(c) illustrates an example of the hybrid IoT network. Due to high mobility and transmit power limitations, not all secondary IoT-nodes are able to communicate with the FC. In this case, they can communicate with the neighboring secondary IoT-nodes in order to send / receive information about channels' availability. Moreover, in case the FC fails the system does not stop working, but transforms into a distributed case.

\subsection{Cooperative Spectrum Sensing Challenges in Cognitive IoT Systems}

The complexity of cognitive IoT systems place numerous challenges for CSS. As discussed before, CR modules for IoT systems are different than those of the conventional ones. While the conventional CR module structure consists of spectrum sensing, spectrum decision, spectrum sharing, and mobility, in the IoT case they include additional entries and, in total, are presented as [3]: spectrum sensing, spectrum analysis, history and prediction, spectrum decision, spectrum-aware routing, security and privacy, spectrum-mobility, and reconfiguration. The addition of novel entries lead to new challenges, which include application-centric processing, mobility, scalability, high integrity, heterogeneity, cooperation and learning, EE, fairness and secondary IoT-node selection, computational complexity, sensing duration and synchronization, interference and user experience. All those challenges are discussed in details in the following sub-sections. 


\subsubsection{Application-Centric Approach / Treatment}

Traditionally, while designing CSS schemes applications are not taken into consideration. However, the context of application support is of great importance to cognitive IoT systems [48], such as smart cities, smart homes, smart grids, wearables, healthcare, connected cars, etc. All those applications of IoT systems are in need of different approaches of bandwidth access, network topologies, mobility, interference tolerance, etc. This means that CSS schemes for cognitive IoT systems should be designed to give a degree of flexibility to be tailored to learn, adapt, and make decisions.

\subsubsection{Mobility of Users}

Depending on the application, the state of users' mobility is different. Some users may have high mobility at all times, others in certain time slots, while several maybe constantly fixed at the same place. In order to achieve successful seamless connectivity in all cases, CSS schemes have to overcome the challenges of adaptive in time and space mobility, spectrum assignment, and sensing and bandwidth allocation in order to be applied to cognitive IoT systems $[6,47]$. Leading by the hardness of satisfying the quality of service (QoS) of application-centric IoT, exploring the nature of mobility directions is a stream that may help in design and decisions of CSS schemes.

\subsubsection{Scalability, High Integrity, and Heterogeneity}

Scalability is the key requirement of any IoT system, which implies network growth with time [7]. However, by adding users, system performance as well as network efficiency and throughput may degrade. Moreover, node types in any IoT system are heterogeneous $[49,50]$. This means that each secondary IoT-node may own a different type of detector, built into it as well as different transmission requirements. The challenge of scalability arises in designing CSS schemes for cognitive IoT systems, such that it is possible to expand the system without the overhead of additional human intervention and overall network performance degradation issues.

\subsubsection{Cooperation and Learning}

NGNs place a focus on learning and feedback information about the environment in cognitive IoT systems by bringing up cooperation of secondary IoT-nodes as an 
essential part of the distributed learning process [4]. Various learning schemes can be used in the distributed CSS process like incremental [51], consensus [52], diffusionbased [53], etc. One of the main tasks is to choose the most appropriate learning scheme, depending on the information available about the network and the characteristics of secondary IoT-nodes. Another main challenges here are the optimal amount of benefit that can be gotten, the amount of iterations needed for the learning process to be completed, and requirements such that the learning process and cooperation can be accomplished.

\subsubsection{Maintaining Energy Efficiency}

Low-power IoT-nodes are not capable of performing spectrum sensing over all channels in cognitive IoT systems due to energy consumption limitations. This is why the amount of channels selected to sense by each user should be limited. After the spectrum sensing process, secondary IoT-nodes can learn from each other by exchanging the information they have. In this way the EE of CSS schemes can be enhanced by reducing the amount of power spent on the sensing process. Furthermore, energy harvesting $(\mathrm{EH})$ can be used as a potential solution for $\mathrm{EE}$ improvement in cognitive IoT systems, where each node can have the characteristics of flexible switching between the operation modes of sensing, harvesting and transmission depending on the time slot and certain network conditions [54].

\subsubsection{Fairness and Cooperative Secondary IoT-nodes Selec- tion}

Cooperation has been validated to be an efficient way of sensing wireless spectrum [12]. However, it creates serious challenges for cognitive IoT systems in terms of fairness in the multi-band channel sensing process [55]. Assuming all channels are equally likely unoccupied by PUs, it is unfair to put more efforts on sensing one channel than others. For example, consuming more power on sensing a certain channel may result in more accurate decisions about the availability of that channel. Nevertheless, power consumption is limited per system, resulting in less power to be consumed on sensing other channels. This leads to performance degradation across other channels as PUs may be mis-detected. In unfair CSS, detection performance may be the same as for the fair case in average. However, detection performance per channel may 
be unacceptable for some of them and very high for others. In contrast, fair CSS provides acceptable detection performance across all channels separately. Hence, it is essential to develop efficient and fair channel sensing and access mechanism in order to have stable system performance across all channels. Moreover, it is required that cooperative secondary IoT-nodes own different information for the learning process to be accomplished. Choosing secondary IoT-nodes with similar outcomes may result in energy loss as no new information can be learned by each one of them. Correct choice of cooperative secondary IoT-nodes may significantly increase system performance, reduce latency as well as the energy consumption of the system.

\subsubsection{Computational Complexity}

In many applications, IoT-nodes are not as computational efficient as central-entities / gateways. Most of the them are low-power and / or low-cost ones with hardware limitations, which leads to limited data storage and processing issues. This brings up the computational simplicity and network efficiency trade-off $[3,12]$. CSS schemes for cognitive IoT systems have to be carefully optimized to address this challenge, while dealing with the cooperative secondary IoT-node selection as well as the sensing process.

\subsubsection{Sensing Duration and Synchronization}

Sensing duration period has always been leading to a trade-off between system reliability and delay, which is critical in delay-sensitive applications [56]. Choosing appropriate sensing time for each application brings reliability and does not delay the process. However, IoT-based systems should be highly integrated, interconnecting various applications altogether, which awakens the challenge of time synchronization. Cooperation in distributed networks requires high synchronization within a channel. How to provide high synchronization and not degrade the performance of the system at the same time is one of the greatest challenges of CSS for distributed cognitive IoT systems.

\subsubsection{Interference Management}

The plethora of IoT devices forms an increasing demand in shared wireless spectrum management in order to avoid interference between PN and CRN. Interference 
sensitive applications are in need of opportunistic spectrum access approach in order to completely avoid interference from the CRN side. However, others may use the underlay or overlay technique, when secondary IoT-nodes are allowed to use the spectrum concurrently with PUs providing certain interference constraints. Furthermore, advances in antenna techniques like adaptive 3D tractable beamforming can be a solution integrated with CSS together to decrease the interference and required transmit power by means of antenna nulling in selected network topologies [3].

\subsection{Summary}

This chapter discussed the three different types of cognitive IoT network architectures: centralized, distributed, and hybrid. Furthermore, it addressed challenges and highlighted important parameters to be considered during the design process of CSS schemes for cognitive IoT systems. From the challenges discussed above it can be concluded that CSS schemes for IoT systems can be carefully improved to further meet heterogeneous, mobile, highly scalable, adaptive, and application-centric IoT systems. Finally, due to the distributed nature of IoT systems, in this thesis, we will focus on distributed multi-band CSS. This is why it is important to study distributed learning approaches to reveal, which one of them can be used in the cognitive IoT scenario. 


\section{Chapter 4}

\section{Distributed Learning Approaches}

\subsection{Introduction}

The role of learning and adaptation is becoming increasingly essential with IoT. Conventionally, learning implies changes within a system that, over time, enables it to perform more effectively within its environment by reusing past experiences [51]. However, we consider learning as an adaptation process. In this thesis, distributed learning implies adaptation in learning through making adjustments in the locally sensed results within each cooperative SU-cluster. Each SU is not only capable of sensing data and experiencing the environment directly, but it also receives information through interactions with its neighboring SUs, processes, and analyzes this information to achieve a common decision about channels' availability [51]. Therefore, from now on, we will be using the term learning to refer to distributed learning adaptation discussed above.

Performance, computational cost, number of iterations needed to reach the consensus point, reliability, immunity to link failures, etc. are different characteristics of learning algorithms, which may vary. Incremental, consensus, and diffusion-based are most commonly used learning schemes in distributed multi-band CRNs [51-53]. It is essential to understand the difference between them, their pros and cons in order to be able to apply them to CSS schemes for cognitive IoT systems. This is why, in this chapter, focus is given to distributed learning approaches named as incremental, consensus, and diffusion.

The rest of the chapter is organized as follows. In Section 4.2, we provide a brief overview of centralized systems' rules in order to point out the difference in detection 
performance measurements between centralized and distributed ways. Further, distributed learning approaches are discussed in Section 4.3, providing the comparison between incremental, consensus, and diffusion-based learning techniques. Section 4.4 illustrates simulation results for distributed learning approaches mentioned above. Finally, a brief summary is given in Section 4.5.

\subsection{Centralized Rules / Algorithms}

Centralized cognitive IoT systems use specific rules to decide on the availability of channels. Traditionally, there are four different centralized rules, which include OR, k-out-of-K, majority, and AND rules [6,37]. In case of the OR rule, the results from all cooperative SUs are obtained according to the OR logic, which implies by itself at least one $\mathrm{SU}$ to detect the PU signal to be present. The k-out-of $\mathrm{K}$ and majority rules decide that the $\mathrm{PU}$ is in the active mode when in total $\mathrm{k}$ out of $\mathrm{K}$ cooperative SUs and the majority of all cooperative SUs detect the PU signal, respectively. As for the AND rule case, all cooperative SUs have to detect the PU signal in order to make a decision that the PU is in the active mode. Otherwise, it is assumed that the channel is not used by the PU.

In case of centralized cooperative multi-band detection, the probability of detection and false alarm for the $m$-th channel become [8]:

$$
\begin{aligned}
& Q_{d, m}=\sum_{q=k}^{K}\left(\begin{array}{c}
K \\
q
\end{array}\right) \times\left\{\prod_{i=1}^{q} P_{d, m i} \times \prod_{j=1}^{K-q}\left(1-P_{d, m j}\right)\right\}, \\
& Q_{f, m}=\sum_{q=k}^{K}\left(\begin{array}{c}
K \\
q
\end{array}\right) \times\left\{\prod_{i=1}^{q} P_{f, m i} \times \prod_{j=1}^{K-q}\left(1-P_{f, m j}\right)\right\} .
\end{aligned}
$$

In case the OR, majority, and AND rules are used, the corresponding $k$ value becomes $1, \frac{K}{2}$ and 0 , respectively. As for the k-out-of-K rule, the value of $k$ will depend on the percentage of SUs needed to detect PU signals, such that channels are believed to be occupied. The corresponding aggregate probability of detection for the CRN can be calculated as:

$$
Q_{d}=\frac{1}{M} \times \sum_{m=1}^{M} Q_{d, m} .
$$




\subsection{Distributed Learning Algorithms}

In contrast to centralized cognitive IoT systems, distributed networks use learning algorithms to decide on the availability of the channel. Cooperative SUs exchange the information sensed and learn from each other in order to reach the consensus value, which corresponds to similar information owning by each cooperative SU. Later, in order to calculate the corresponding aggregate probability of detection, the results of detection probabilities of all channels are averaged in a similar manner to the centralized case, as specified in (4.2). In this section, we are focusing on the learning process. The comparison between three different learning approaches, named as incremental, consensus, and diffusion-based learning, is provided to discover which one of them best suits the IoT demands.

\subsubsection{Incremental-based Approach}

The incremental approach can be implemented in a connected network, where using a certain trajectory, sensing nodes are being visited one by one [51]. Learning can be performed only by one user at a time. In particular, $k$-th secondary IoT-node combines a small portion of a previously visited cooperative secondary IoT-node's test statistics information, $S_{j}^{m}$, with its own, $S_{k}^{m}$, at each iteration $i$ with $\mu_{k}^{m}$ as a learning step size for the $m$-th channel as follows:

$$
S_{k}^{m}(i+1)=S_{k}^{m}(i)+\mu_{k}^{m} \times\left(S_{j}^{m}(i)-S_{k}^{m}(i)\right)
$$

Completing one cycle means going one by one through all secondary IoT-nodes in a network trajectory and complies completing one iteration. By visiting each sensing node, a portion from the previously visited node's information is being interchanged.

One of disadvantages of this learning mechanism is the mandatory trajectory creation. Cooperative secondary IoT-nodes have to form kind of a ring topology within themselves in order to be able to exchange the information and learn. In case of one of the users fails, the whole learning process is left unaccomplished due to disruption of this virtual ring topology. Thus, the mechanism has to be repeated over again. Moreover, learning only from one user at a time instance is inefficient and leads to high system delays, which is critical in delay sensitive applications, especially when the system is growing in size. This brings difficulties in the ability of the following algorithm to be used for scalable systems. 


\subsubsection{Consensus-based Approach}

Consensus-based mechanism appears to be more appealing for cognitive IoT systems than the incremental one. This comes from the way the algorithm is accomplished. Here, neighboring cooperative secondary IoT-nodes exchange sensed information with each other at once. In particular, $k$-th secondary IoT-node combines a small portion of all other cooperative secondary IoT-nodes' test statistics information with its own at each iteration $i$ in the case of $Q_{m}$ cooperative secondary IoT nodes with $\mu_{k}^{m}$ as a learning step size for the $m$-th channel as follows [52]:

$$
S_{k}^{m}(i+1)=S_{k}^{m}(i)+\mu_{k}^{m} \times \sum_{\substack{j=1, j \neq k}}^{Q_{m}}\left(S_{j}^{m}(i)-S_{k}^{m}(i)\right)
$$

This means that the process represents a certain virtual mesh topology, which leads to the fact that the system is more stable in case of link failures. Finally, due to the fact that all secondary IoT-nodes are updated simultaneously, the consensus process itself is faster by a factor of the total cooperative secondary IoT-nodes number for a selected channel in the system than the incremental one. This means that the algorithm can be implemented for scalable IoT systems.

\subsubsection{Diffusion-based Approach}

Another learning algorithm for distributed networks is based on diffusion adaptation [53]. As discussed previously, in consensus, each secondary IoT-node uses its own information as the base point. This leads to the asymmetry in the learning process among all users. However, the diffusion-based algorithm addresses the issue of asymmetry in learning processes of the incremental and consensus cases. This is revealed as the only difference in implementation of consensus and diffusion-based algorithms. In the diffusion case, secondary IoT-node's test statistics information is being replaced by the weighted average test statistics information of all cooperative secondary IoT-nodes, which is defined as follows:

$$
S_{\text {avg }}^{m}(i+1)=\sum_{j=1}^{Q_{m}} \alpha_{j}^{m} \times S_{j}^{m}(i),
$$

where $S_{j}^{m}$ is the test statistics of each $j$-th cooperative SU for the $m$-th channel and 
$\sum_{j=1}^{Q_{m}} \alpha_{j}^{m}=1$. In particular, $k$-th secondary IoT-node combines a small portion of all other cooperative secondary IoT-nodes' test statistics information with the weighted average of all test statistics at each iteration $i$ in the case of $Q_{m}$ cooperative secondary IoT nodes with $\mu_{k}^{m}$ as a learning step size for the $m$-th channel as follows:

$$
S_{k}^{m}(i+1)=S_{\text {avg }}^{m}(i)+\mu_{k}^{m} \times \sum_{\substack{j=1, j \neq k}}^{Q_{m}}\left(S_{j}^{m}(i)-S_{\text {avg }}^{m}(i)\right) .
$$

The use of the weighted average of cooperative users' test statistics brings symmetry in learning in addition to making the learning process much faster, as will be seen in the next sections.

Table 4.1: Comparison of incremental, consensus, and diffusion-based learning approaches' characteristics.

\begin{tabular}{|c|c|c|c|}
\hline Characteristics & Incremental & Consensus & Diffusion \\
\hline \hline $\begin{array}{c}\text { Link failure } \\
\text { case }\end{array}$ & $\begin{array}{c}\text { Learning process } \\
\text { interrupted }\end{array}$ & $\begin{array}{c}\text { In some cases } \\
\text { may not converge }\end{array}$ & $\begin{array}{c}\text { Converges } \\
\text { always }\end{array}$ \\
\hline $\begin{array}{c}\text { Single iteration } \\
\text { node execution }\end{array}$ & $\begin{array}{c}\text { One by } \\
\text { one }\end{array}$ & $\begin{array}{c}\text { All nodes } \\
\text { simultaneously }\end{array}$ & $\begin{array}{c}\text { All nodes } \\
\text { simultaneously }\end{array}$ \\
\hline Scalability & Non-scalable & Scalable & Scalable \\
\hline Speed & Very slow & Fast & Super fast \\
\hline $\begin{array}{c}\text { Complexity per } \\
\text { single iteration }\end{array}$ & Low & Medium & Relatively high \\
\hline Total complexity & High & Medium & Low \\
\hline $\begin{array}{c}\text { Energy } \\
\text { consumption }\end{array}$ & Very high & Medium & Low \\
\hline $\begin{array}{c}\text { Difference in } \\
\text { consensus point }\end{array}$ & $\begin{array}{c}\text { Same final } \\
\text { convergence point }\end{array}$ & $\begin{array}{c}\text { Same final } \\
\text { convergence point }\end{array}$ & $\begin{array}{c}\text { Same final } \\
\text { convergence point }\end{array}$ \\
\hline $\begin{array}{c}\text { Symmetry in } \\
\text { learning }\end{array}$ & Very low & Medium & High \\
\hline
\end{tabular}




\subsubsection{Comparison}

The summarized characteristics' comparison of three different algorithms in terms of link failure immunity, single iteration node execution, scalability, speed, complexity per single iteration, total complexity, energy consumption, difference in the final consensus point result, and symmetry in learning are exposed in Table 4.1.

For the diffusion-based approach, in case of a link failure, the process is never interrupted and always converges, which cannot be offered by either incremental or consensus approaches. This can be justified as follows. In case of link failures, the incremental algorithm will not converge because the virtual link topology is broken. As for the consensus algorithm, the algorithm does not converge, when the noise level over a certain number of sensed samples is very high. However, the appeared high noise level fades due to the averaging for the diffusion-based case, leading to persistent convergence. This impacts the stability of the system and reveals diffusion-based algorithm as the most stable of all discussed above.

In addition to this, as the number of cooperative secondary IoT-nodes increases, the time needed to perform one iteration rises up for the incremental approach. This is why it is believed to be non-scalable, which is critical for IoT systems. On contrast, consensus and diffusion-based algorithms need much less time to perform the learning process. For instance, if the time needed for the consensus algorithm per iteration is $T_{\text {learning,cons }}$, the corresponding incremental learning process will take the following amount of time:

$$
T_{\text {learning, }, \text { inc }}=T_{\text {learning,cons }} \times Q_{m} .
$$

Table 4.2: Comparison of incremental, consensus, and diffusion-based learning approaches' complexity on sensing the $m$-th channel per one iteration.

\begin{tabular}{|c|c|c|c|}
\hline Characteristics & Incremental & Consensus & Diffusion \\
\hline \hline $\begin{array}{c}\text { Number of } \\
\text { additions }\end{array}$ & $2 \times Q_{m}$ & $\left(Q_{m}\right)^{2}$ & $2 \times\left(Q_{m}\right)^{2}$ \\
\hline $\begin{array}{c}\text { Number of } \\
\text { multiplications }\end{array}$ & $Q_{m}$ & $Q_{m}-1$ & $\left(Q_{m}\right)^{2}+Q_{m}-1$ \\
\hline
\end{tabular}


This can be explained by the fact that the consensus algorithm performs learning in parallel for all nodes, while the incremental algorithm does it node by node. In addition to this, although due to the averaging in the diffusion-based learning the time taken by one iteration is slightly higher than for the consensus algorithm, the diffusion learning is much faster in total.

Moreover, computational complexity is another factor of interest. Computational complexity is illustrated in the number of additions and multiplications in Table 4.2. Although, the diffusion algorithm brings higher computational complexity per iteration than incremental and consensus-based algorithms, in total, it requires the lowest number of iterations needed to reach the consensus point. This is why the diffusionbased distributed cognitive IoT system requires less cost and energy to be consumed during the learning process than the incremental or consensus-based systems. It represents an advantage for IoT devices with certain hardware limitations and low power consumption.

Finally, as it will be seen from simulation results provided in Section 4.4, all three learning schemes eventually lead to the same final convergence point in case similar learning step sizes used.
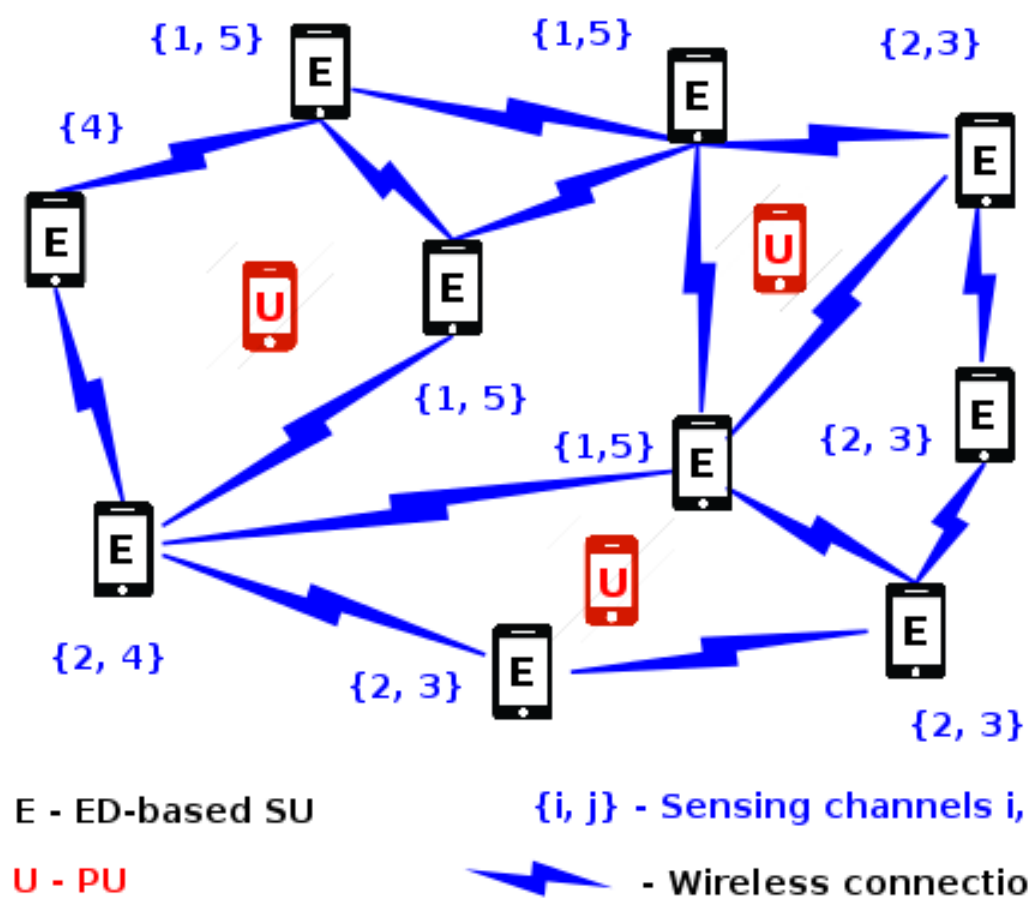

$\{\mathrm{i}, \mathrm{j}\}$ - Sensing channels $\mathrm{i}, \mathrm{j}$

$\cup-P \cup$

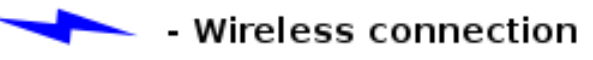

Figure 4.1: Homogeneous distributed ED-based topology. 


\subsection{Simulation Results}

In this section, we are analyzing incremental, consensus, and diffusion-based learning approaches through simulation results. In order to compare and decide, which learning processes from the discussed above can be used for cognitive IoT systems, convergence rate and scalability results are illustrated and compared.

The case of distributed homogeneous ED-based system scenario of $K=10$ secondary IoT nodes and $M=5$ channels is considered, as illustrated in Fig. 4.1. All

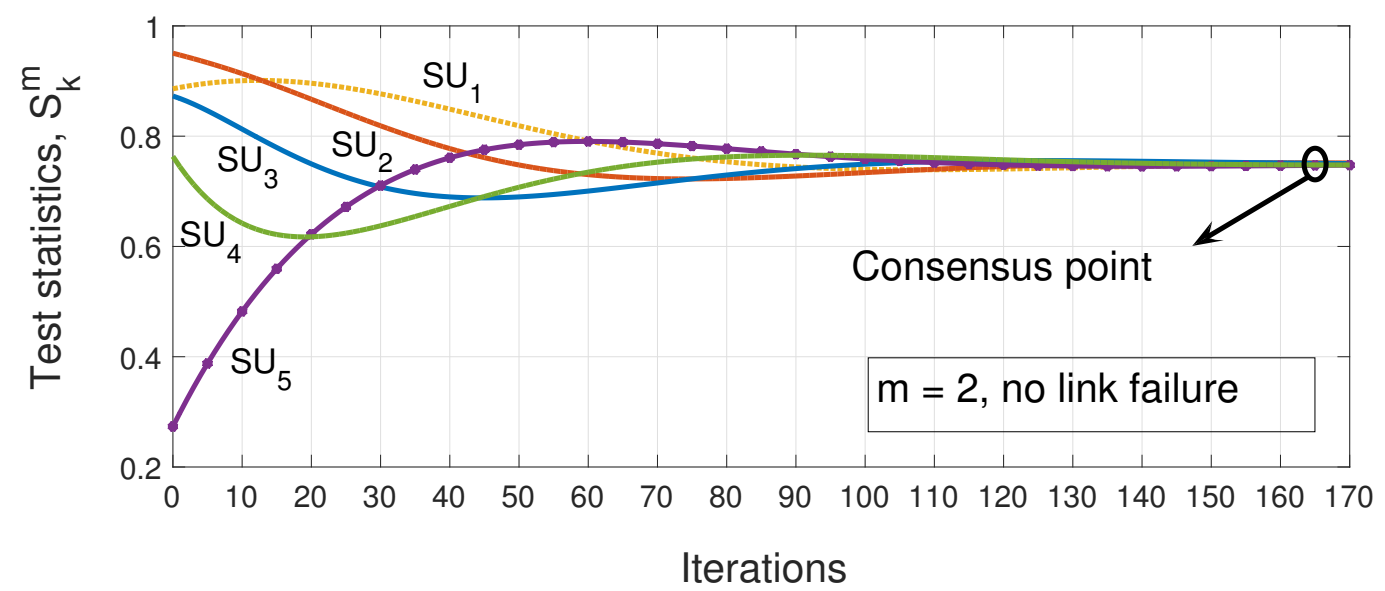

(a)

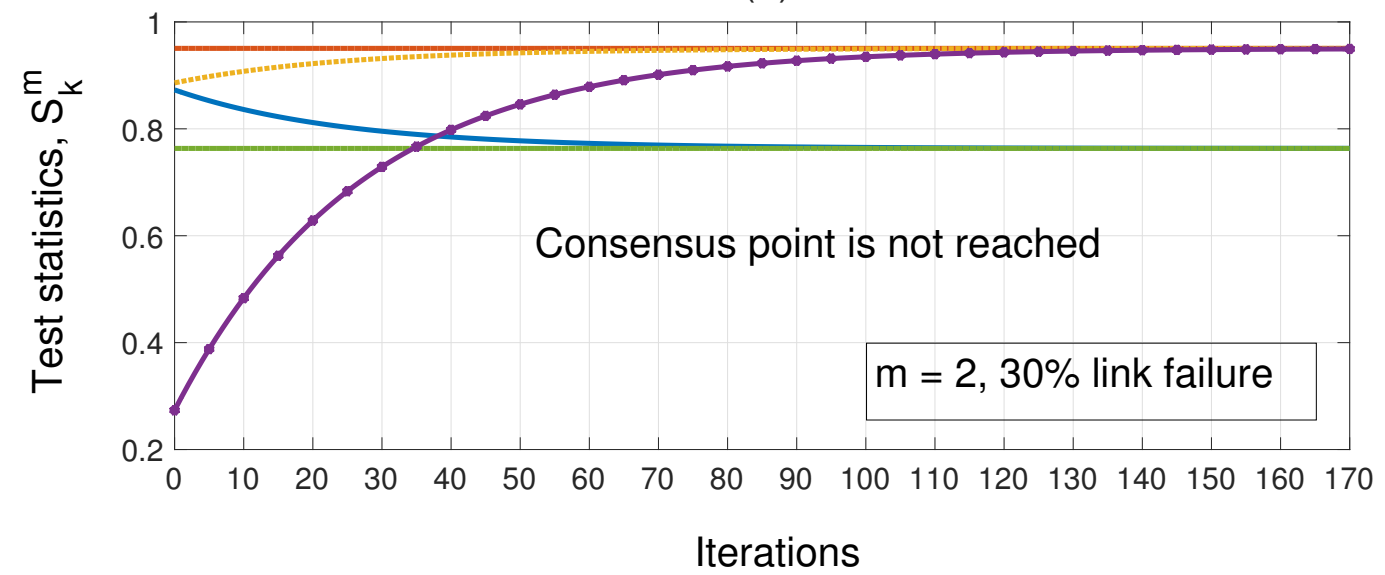

(b)

Figure 4.2: Incremental-based learning curves of five cooperative secondary EDbased IoT-nodes sensing channel 2: (a) without the link failure and (b) with the $30 \%$ link failure. 
secondary IoT-nodes are equipped with only one detector due to hardware limitations. It is also assumed that no prior information is available about channels. Each $\mathrm{SU}$ is assumes to sense a maximum of $I=2$ channels. Cooperative SUs are selected arbitrary in this case, using the RSSS scheme [36].

To begin with, the speed of convergence without and with the link failure for the incremental, consensus, and diffusion-based learning approaches are performed as shown in Figs. 4.2, 4.3, and 4.4, respectively. For the link failure case, 30\% of links are assumed to be failed in total. The results are revealed for channel 2. Five

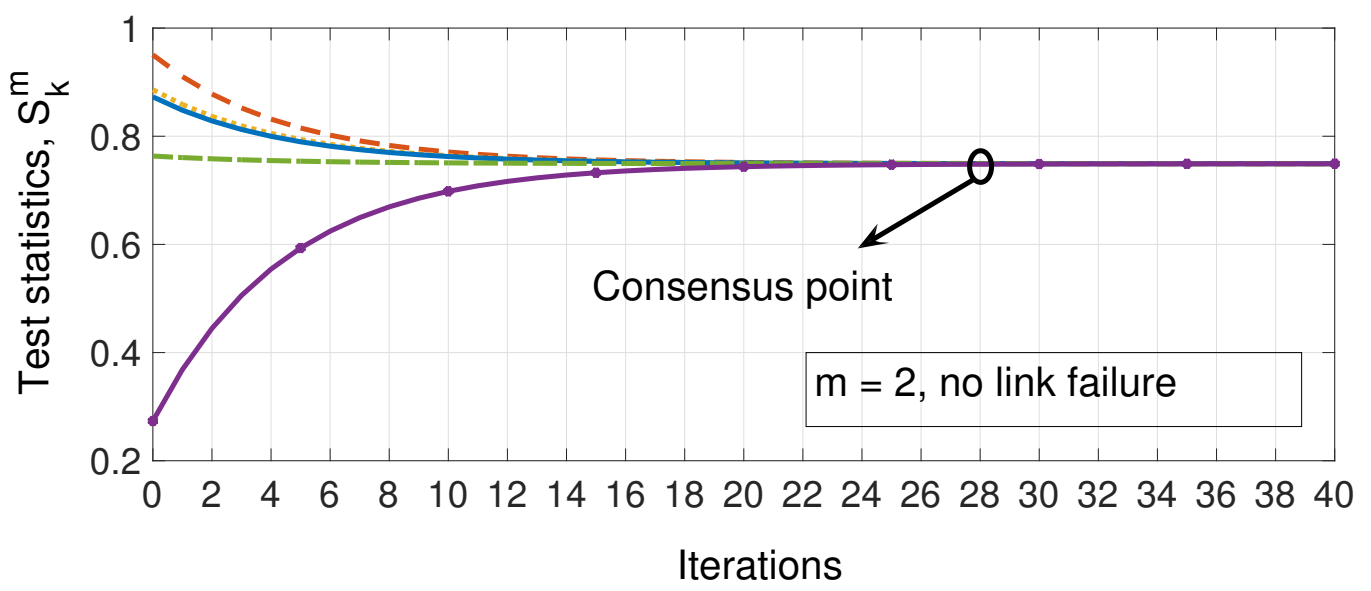

(a)

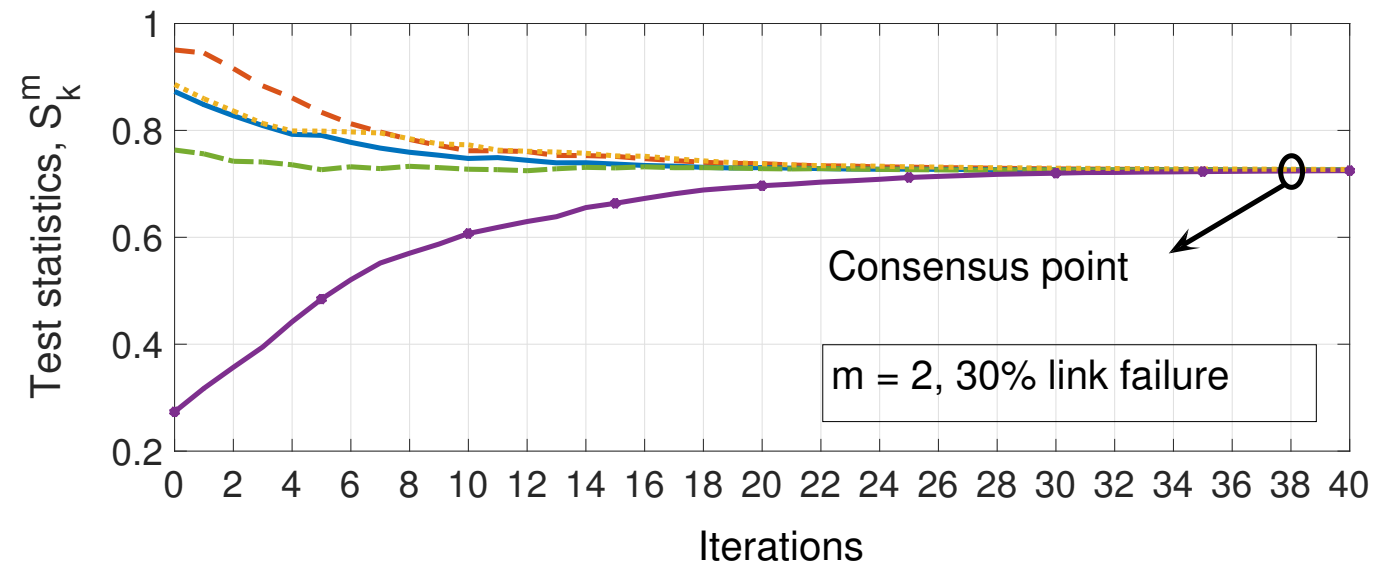

(b)

Figure 4.3: Consensus-based learning curves of five cooperative secondary ED-based IoT-nodes sensing channel 2: (a) without the link failure and (b) with the $30 \%$ link failure. 
secondary ED-based IoT-nodes interchange locally sensed information and after a certain amount of time the consensus point is reached, such that the process can be interrupted at that point and the decision made.

As shown in Fig. 4.2(a), 165 iterations were required to reach the consensus point for the incremental-based algorithm. Moreover, in case of the link failure of Fig. 4.2(b), the incremental-based learning, as was expected, is highly inefficient. The algorithm does not converge, if the virtual ring topology between cooperative SUs is disrupted. As for the consensus-based learning algorithm, 28 iterations were required to reach the consensus value in Fig. 4.3(a). On condition of the link failure,

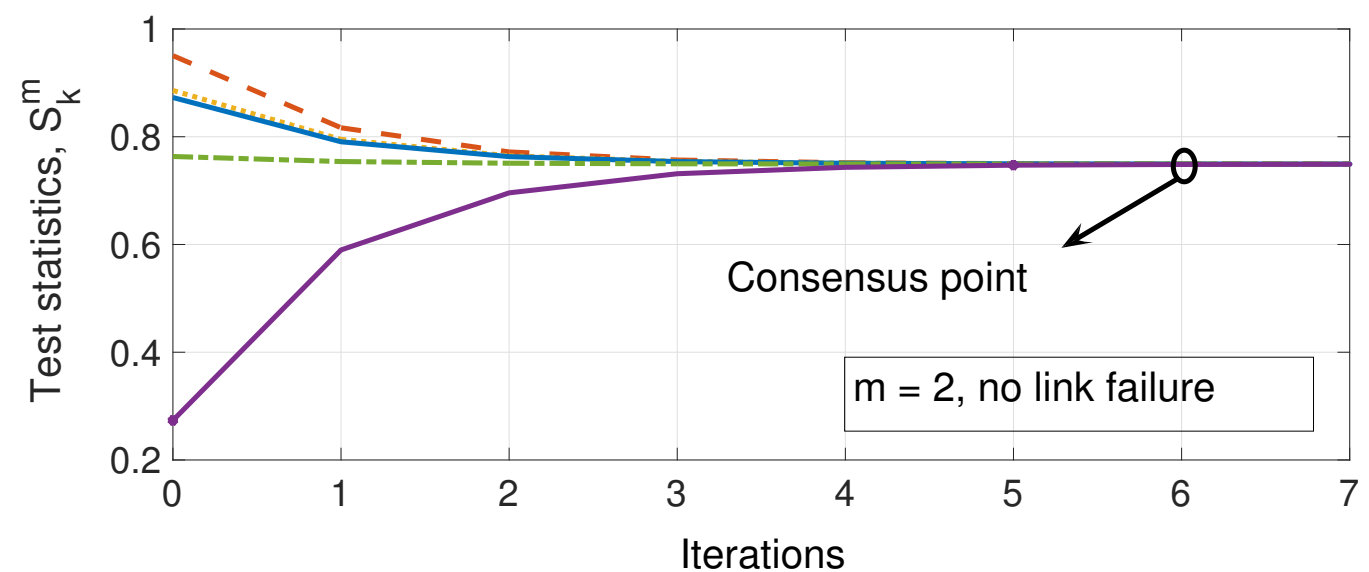

(a)

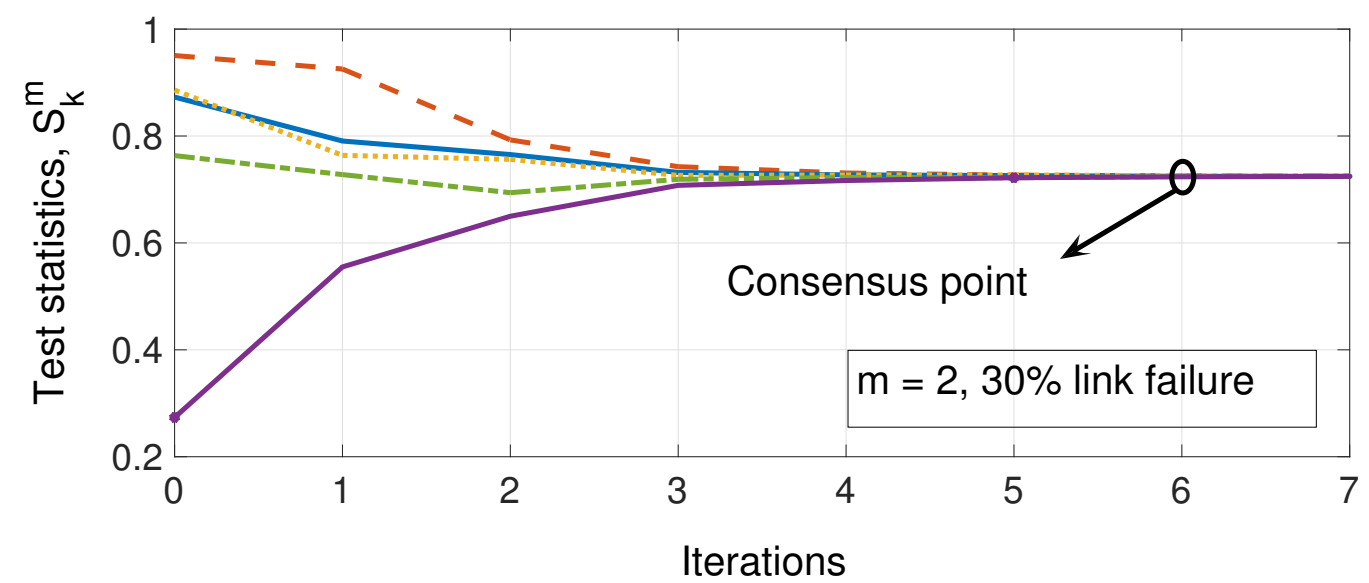

(b)

Figure 4.4: Diffusion-based learning curves of five cooperative secondary ED-based IoT-nodes sensing channel 2: (a) without the link failure and (b) with the $30 \%$ link failure. 
the consensus-based algorithm converges, yet, extra iterations are needed to reach the consensus value as displayed in Fig. 4.3(b). In contrast, the diffusion-based algorithm required only 6 iterations to fulfill the learning process as shown in Fig. 4.4(a). Moreover, it presented high efficiency in terms of stability. Given the link failure case of Fig. 4.4(b), the system not only converges to one single consensus value, but also does not require any extra number of iterations to do so. This advantage of the diffusion-based algorithm can be explained by the averaging process performed at each iteration.

It can be observed that all three algorithms come to the same consensus point resulting value in case of no link failure. However, more than double improvement

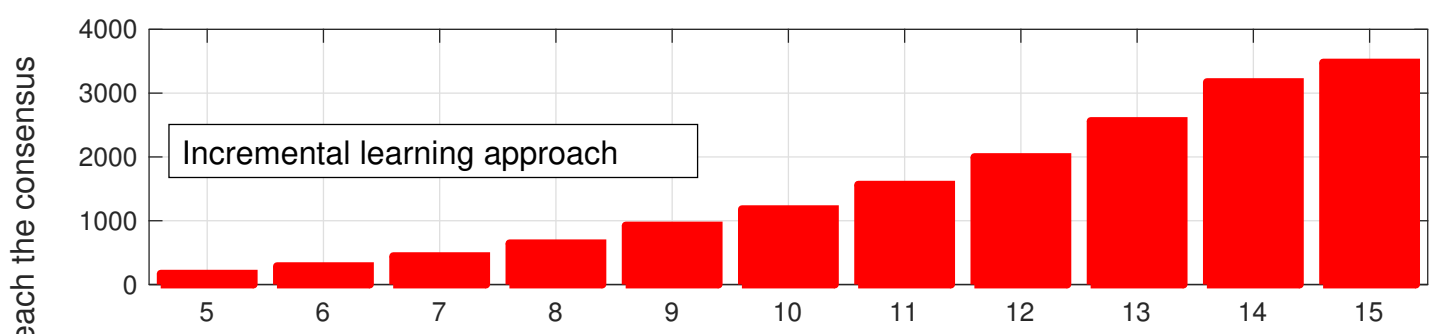

(a)

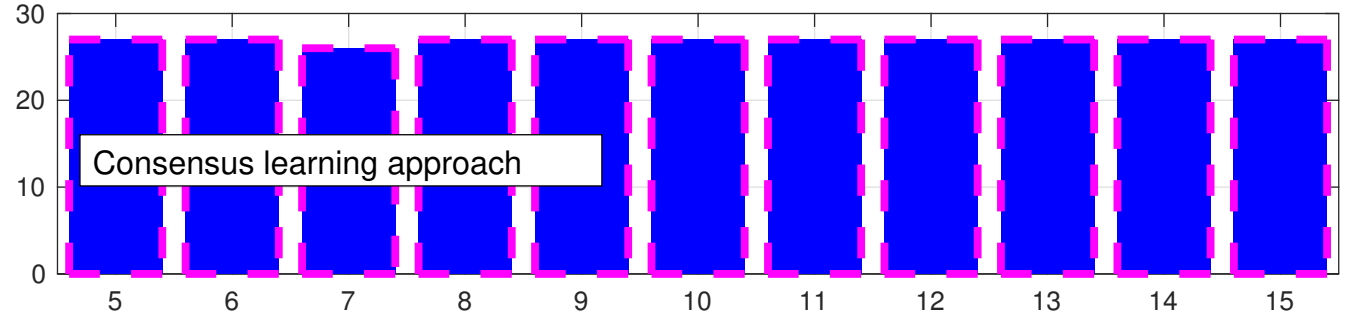

(b)

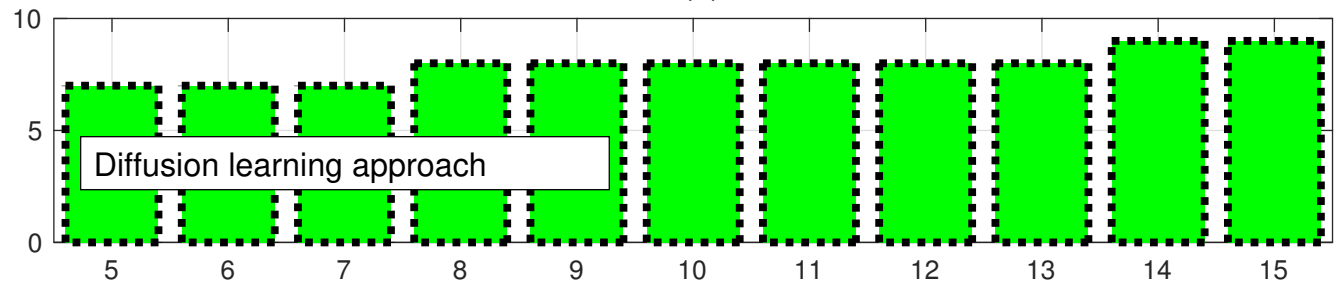

(c)

Number of cooperative secondary loT-nodes

Figure 4.5: Scalability results, average number of iterations needed to reach a consensus point for different values of cooperative secondary IoT-nodes using (a) incremental, (b) consensus, and (c) diffusion-based learning algorithms. 
in convergence rate is achieved when compared incremental with consensus and consensus with diffusion algorithms. This means that the diffusion algorithm needs less number of iterations to reach consensus and proves the diffusion-based algorithm to be the quickest in terms of speed.

In addition to this, simulation results illustrating the average amount of iterations needed to reach the consensus as the number of cooperative secondary IoT nodes grow are conveyed in Fig. 4.5. For this purpose, we add new SUs to the system. We also assume that the new nodes join the cooperative SU-cluster of channel 2. It is clear that for the incremental approach as the number of cooperative secondary IoT-nodes increase, the number of iterations needed to terminate the learning process, in other words reach the consensus point, grows. This means that scaling up the system based on the the incremental learning approach is not a good idea as the amount of energy and time needed on the learning process increases together with the network size. This explains why the algorithm is not the best choice for growing IoT systems. To address the scalability issue of cognitive IoT systems, both consensus and diffusionbased approaches appear to be efficient solutions. Figs. 4.5(b) and (c) illustrate that increasing the number of cooperative secondary IoT-nodes does not affect the amount of iterations needed for the process to be accomplished for both algorithms, which means no systematic delay or extra energy consumption per user. Moreover, it is clear from Fig. 4.5 that compared with the incremental approach, even at very low values of cooperative secondary IoT-nodes, the amount of iterations needed to reach the consensus is much less for both consensus and diffusion-based approaches. It can be concluded that consensus and diffusion algorithms can be successfully used in cognitive IoT systems. However, the diffusion-based algorithm has proved to be more efficient than the consensus one in terms of link failure stability and number of iterations needed to reach the consensus point. This is why from now on, the diffusion-based algorithm will be used in this thesis.

\subsection{Summary}

We provided the analysis of different learning schemes as incremental, consensus, and diffusion-based for distributed CRNs. After analyzing the aforementioned algorithms from different prospectives, the diffusion-based algorithm can be concluded to be most promising one concerning the IoT system demands. 
Finally, enabling breadth into IoT spectrum usage can be a hard task to achieve, however, multi-band CSS is believed to be an efficient way of doing that. By carefully designing and developing CSS schemes, billions of devices can communicate in future cognitive IoT systems. Efficient and reliable CSS schemes are required for accessing spectrum opportunistically. In the next chapter, we propose a novel CSS scheme, named as HM2CSS. 


\section{Chapter 5}

\section{Heterogeneous Multi-band Multi-user Cooperative Spectrum Sensing}

\subsection{Introduction}

In this chapter, we propose a novel CSS scheme, called HM2CSS. As it was stated before, HM2CSS stands for heterogeneous multi-band multi-user CSS. We select cooperative SUs for distributed multi-band CSS in two stages. In the first stage, we formulate an optimization problem to select an SU-leader for each channel. We then formulate another optimization problem to select corresponding cooperative SUs to perform local spectrum sensing for each channel in the second stage. After cooperative SU-selection is made, the sensing process takes place and using diffusion-based learning algorithm, cooperative SUs exchange sensed information in order to determine the availability of channels. The proposed scheme is designed in a way to enable scalability and improve detection performance, enhance CRN throughput, and provide fair energy consumption for spectrum sensing on all channels.

The rest of the chapter is organized as follows. In Section 5.2, system model is discussed. We investigate the proposed HM2CSS scheme thoroughly in Section 5.3. In Section 5.4, extensive simulation results are presented. The proposed distributed HM2CSS scheme is compared to existing distributed and centralized schemes. Finally, conclusions are made in Section 5.5. 


\subsection{System Model}

We investigate a distributed heterogeneous $\mathrm{CRN}$, which consists of $M$ channels, i.e., $\mathcal{M}=\left\{m_{1}, m_{2}, \ldots, m_{M}\right\}$, owned by PUs and $K$ SUs, i.e., $\mathcal{K}=\left\{S U_{1}, S U_{2}, \ldots, S U_{K}\right\}$, with $M<K$. Each PU is assumed to be either in an active or idle mode. SUs sense channels cooperatively and decide whether they are occupied by PUs or not. For this purpose, each $\mathrm{SU}$ is assumed to be equipped with one detector, such that in total there are $K^{E} \mathrm{ED}, K^{P} \mathrm{PD}$, and $K^{O}$ OD-based SUs as illustrated in Fig. 5.1, i.e., $K=K^{E}+K^{P}+K^{O}$. The SNR value for each SU sensing the $m$-th channel is random with the fixed average SNR value for all channels. Communication is assumed to be performed over the Rayleigh fading channel.

As shown in Fig. 5.1, only certain SUs in the CRN can communicate with each

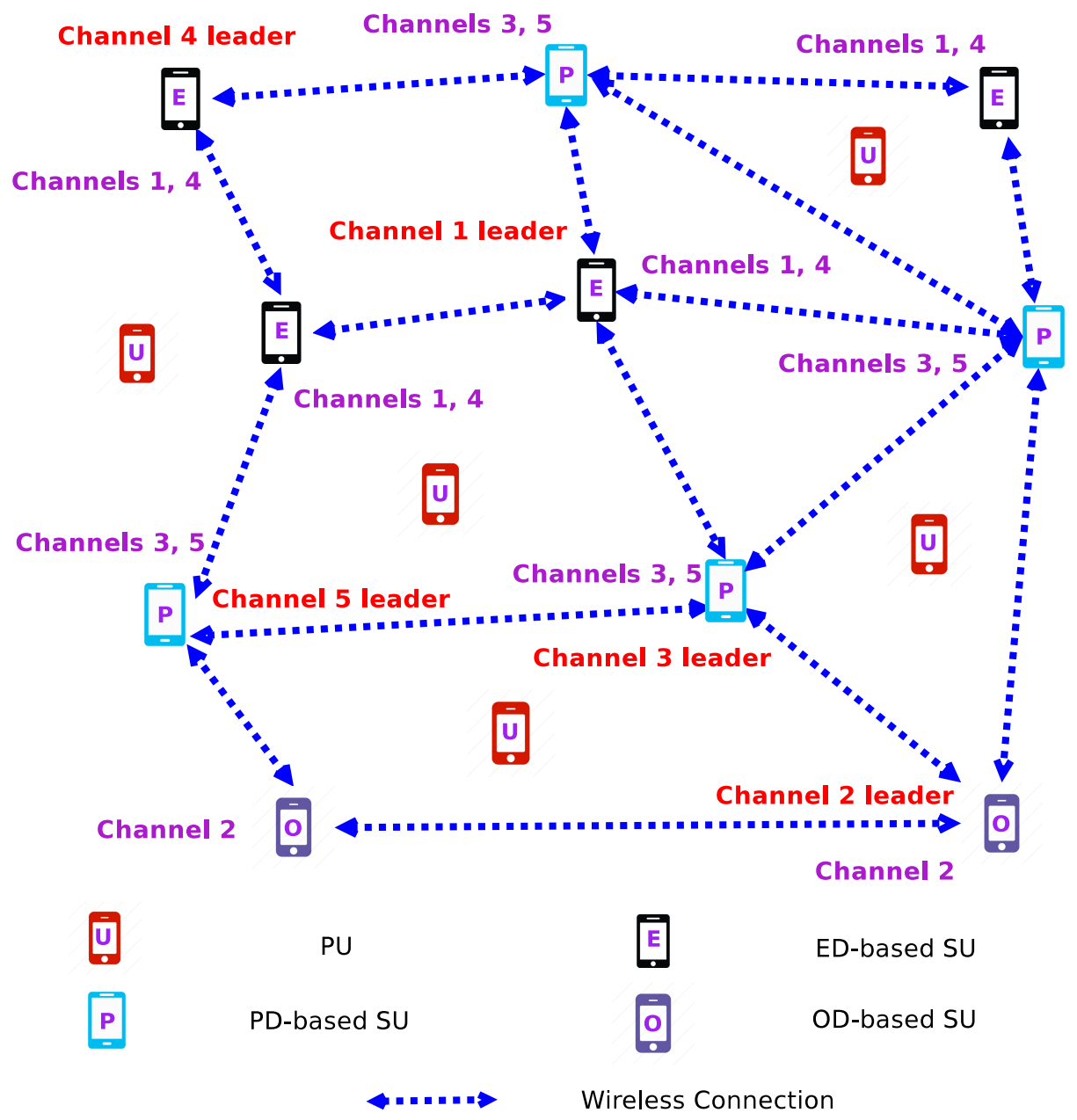

Figure 5.1: Heterogeneous distributed multi-user CRN topology. 
other. This is because of the geographic location and fading environment. However, all SUs participate in the CSS process. Each $k$-th SU performs local spectrum sensing using $N_{m}^{k}$ samples for each channel $m \in \mathcal{M}$ assigned to it. Then, cooperative SUs can exchange their sensing information to determine the occupancy of channels. It is assumed that each SU can sense minimum one and maximum $I$ channels, where $I<M$. In addition, some of SUs are chosen to fulfill the role of SU-leaders for channels, which involves assisting in cooperative SU-selection for each channel.

Depending on the information available about a channel, certain SUs are chosen to sense it. Three main channel types considered are with no prior information about the PU signal, with known pilot tone of the PU signal, and the channel with an OFDM PU signal being sent over it. We assume that $M^{N}$ is the total number of channels with no information available about them, $M^{P}$ is the total number of channels with the known pilot tones, and $M^{O}$ is the total number of channels known to carry an OFDM signal. This means that, $M=M^{N}+M^{P}+M^{O}$. Based on this information, SUs are divided into cooperative SU-clusters, which sense the assigned channels. In cases no information is known about the channel, the pilot tone of the channel's signal is known, and the signal of the channel is known to be an OFDM one, the ED, PD, and OD-based users are selected to sense the channel, respectively. Finally, we assume that the system contains sufficient amount of SUs to detect each channel type.

\subsection{Heterogeneous Multi-band Multi-user Coop- erative Spectrum Sensing}

A flow chart for the proposed HM2CSS scheme is represented in Fig. 5.2. The inputs for the proposed HM2CSS scheme are $K$ heterogeneous SUs, containing $K^{E}$ $\mathrm{ED}, K^{P} \mathrm{PD}$, and $K^{O}$ OD-based sensors and $M$ channels, where each SU can sense maximum $I$ channels, i.e., $I<M$. The available information about all channels is different. According to the information present about channels, the first stage of the proposed HM2CSS scheme is to select an SU-leader for each channel $m \in \mathcal{M}$. In case no information is available about the PU signal, i.e., $m^{N} \in M^{N}$, the SU-leader is selected from the ED-based SUs subset, $\mathcal{K}^{\mathcal{E}} \in \mathcal{K}$. If the pilot tone is known, i.e., $m^{P} \in M^{P}$, the SU-leader is selected from the PD-based SUs subset, $\mathcal{K}^{\mathcal{P}} \in \mathcal{K}$. Otherwise, if the signal sent by the PU across the channel is believed to be the 


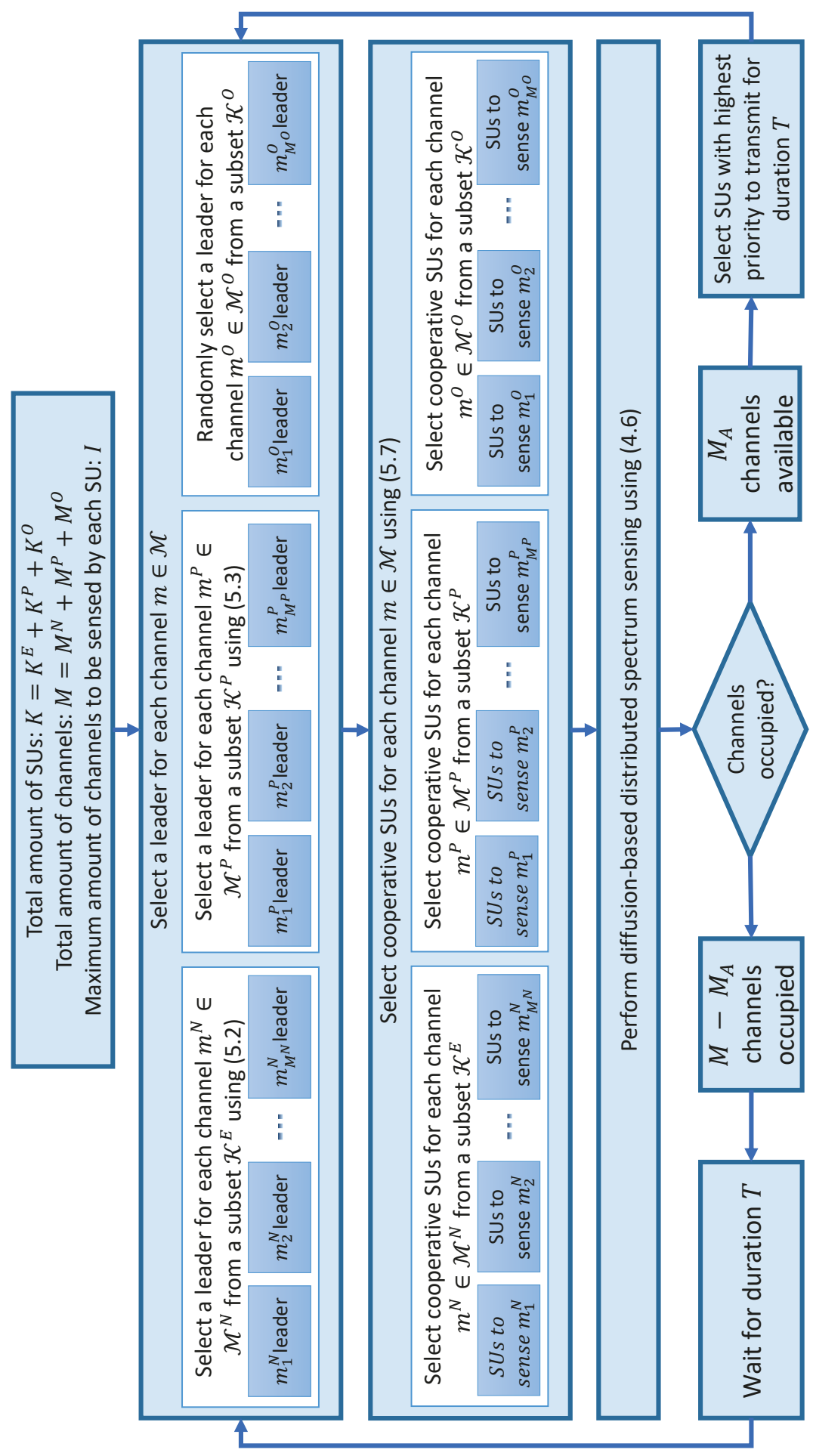

Figure 5.2: A flow chart for the proposed HM2CSS scheme. 
OFDM one, i.e., $m^{O} \in \mathcal{M}^{\mathcal{O}}$, then the leader is selected from the OD-based SUs subset, $\mathcal{K}^{\mathcal{O}} \in \mathcal{K}$. After that, the CVM goodness-of-fit test together with the SUleaders chosen are used to select cooperative SUs to sense each channel $m \in \mathcal{M}$ as a second stage of the proposed HM2CSS scheme. Similarly to the leaders' choice, the cooperative SU-selection depends on the information available about the channels, such that in cases no prior information about the signal, the known pilot tone of the signal, the signal is known to be OFDM, cooperative SUs are chosen to be from the ED, PD, OD-based SUs subsets, respectively. This is necessary in order to provide synchronization to the learning process and use the most out of information available for sensing. The two stages mentioned above are discussed in details in the following sub-sections. After the cooperative SU-selection process is accomplished, diffusionbased distributed learning is performed by each cooperative SU-cluster to determine whether the channels are occupied or not. Those channels, which are determined to be in the idle mode, are available for SUs' transmission, meaning that SUs with highest priorities to use channels can transmit for the time duration $T$. As for the occupied channels, SUs are not allowed to use them. Thus, they have to wait for time duration $T$ and then, repeat the process from the beginning.

\subsubsection{Stage 1: Leader Selection}

Optimal leader selection implies determining the best SU to listen to for each channel $m$. Leaders will be responsible to select cooperative SUs for distributed spectrum sensing. This is why it is essential to make the correct choice of a leader for each channel. We can define a binary indicator $b_{m k}$ to show whether the $k$-th $\mathrm{SU}$ is chosen as a leader for the $m$-th channel or not:

$$
b_{m k}= \begin{cases}1, & \text { if } k-t h \mathrm{SU} \text { is selected as a leader for channel } m \\ 0, & \text { otherwise. }\end{cases}
$$

\subsubsection{Energy Detector Based Leader Selection}

Due to the fact that ED-based detection is done by estimating the received energy level across the channel, whether the noise level is high or low, together with the PU signal it is detected. In case the noise level is high and the PU is in the idle mode, an ED-based SU may decide that the PU is present. This is why the SNR value of the 
channel plays an important role in this type of detection. Let $S N R_{\text {avg }}$ be the average SNR value across each channel. In order to select cooperative SUs with both higher and lower SNR values, the leader of channel $m^{N} \in \mathcal{M}^{\mathcal{N}}$ should have low correlation in its signal envelope with the signal envelopes of its cooperative SUs. This is why for channels with no prior information available about them, the objective is to select leaders with the smallest difference between $S N R_{m^{N} k^{E}}$ and $S N R_{\text {avg }}$ values, i.e., SUs with SNR close to $S N R_{\text {avg. }}$. The optimization problem formulation to select leaders for $m^{N} \in \mathcal{M}^{\mathcal{N}}$ channels can be defined as follows:

$$
\begin{aligned}
\min _{b_{m^{N}} E}: & \sum_{k^{E}=1}^{K^{E}} \sum_{m^{N}=1}^{M^{N}} b_{m^{N} k^{E}} \times\left|S N R_{m^{N} k^{E}}-S N R_{a v g}\right|, \\
\text { s.t. } & C 1: \sum_{m^{N}=1}^{M^{N}} b_{m^{N} k^{E}}=c_{k}^{E}, \forall k^{E} \in \mathcal{K}^{\mathcal{E}}, \\
& C 2: \sum_{k^{E}=1}^{K^{E}} b_{m^{N} k^{E}}=1, \forall m^{N} \in \mathcal{M}^{\mathcal{N}},
\end{aligned}
$$

where $c_{k}^{E}$ is the binary value, which equals to 1 if $S U_{k}^{E}$ is a leader of one of the channels from a subset of $\mathcal{M}^{\mathcal{N}}$ and 0 otherwise. C1 ensures that same SU cannot be selected as a leader for more than one channel. Whereas $\mathrm{C} 2$ confirms that only one leader can be selected for each channel.

\subsubsection{Pilot-tone Detector Based Leader Selection}

For PD-based sensed channels, the orthogonality property between the pilot tone and the data-carrying part of the signal is used for detection purposes. This is why noise existence here is not as crucial as in the ED-based case. Due to the fact that we own the information about the pilot tone of the PU signal, we require such a leader that we can easily recognize the pilot tone in it. Hence, a leader for each channel $m^{P} \in \mathcal{M}^{\mathcal{P}}$ is selected with highest SNR value. The optimization problem formulation for PD-based leader selection can be defined as follows: 


$$
\begin{aligned}
\max _{b_{m^{P}} k^{P}}: & \sum_{k^{P}=1}^{K^{P}} \sum_{m^{P}=1}^{M^{P}} b_{m^{P} k^{P}} \times S N R_{m^{P} k^{P}}, \\
\text { s.t. } & C 1: \sum_{m^{P}=1}^{M^{P}} b_{m^{P} k^{P}}=c_{k}^{P}, \forall k^{P} \in \mathcal{K}^{\mathcal{P}}, \\
& C 2: \sum_{k^{P}=1}^{K^{P}} b_{m^{P} k^{P}}=1, \forall m^{P} \in \mathcal{M}^{\mathcal{P}},
\end{aligned}
$$

where $c_{k}^{P}$ is the binary value, which equals to 1 if $S U_{k}^{P}$ is a leader of $k$-th channels and 0 otherwise. $\mathrm{C} 1$ ensures that same $\mathrm{SU}$ cannot be selected as a leader for more than one channel. Whereas $\mathrm{C} 2$ confirms that only one leader can be selected for each channel.

\subsubsection{Orthogonal Frequency Division Multiplexing Detector Based Leader Selection}

As for the OD-based detection, using the knowledge of cyclic prefix and OFDM symbol length, the inherent signal correlation incurred by cyclic prefix repetition is used to detect the PU signal. Due to the fact that sufficient amount of information is available about the PU signal, there is no need for the specific leader choice or an optimization problem formulation as it only increases the complexity of the algorithm in this case. Hence, this property of the OD-based scenario allows us to randomly select a leader for each channel $m^{O} \in \mathcal{M}^{\mathcal{O}}$ from the subset of $\mathcal{K}^{\mathcal{O}}$ SUs.

It is essential to note that for all three channel types mentioned above, the SUleader selection is independent and performed concurrently. This comes from the fact that only ED-based SUs may be selected as leaders for each channel $m^{N} \in \mathcal{M}^{\mathcal{N}}$, PD-based SUs for each channel $m^{P} \in \mathcal{M}^{\mathcal{P}}$, and OD-based SUs for each channel $m^{O} \in \mathcal{M}^{\mathcal{O}}$. However, it is clear from (5.2) and (5.3) that leader selection among channels of the same type is dependent as only one SU-leader is allowed per channel, and the same SU cannot be a leader for more than one channel.

Finally, the problems in (5.2) and (5.3) are of mixed integer linear programming (MILP) type. Each one of them includes a linear objective function and constraints. In both problems, all variables are binary. To solve these problems, we adopted the B\&B algorithm [57]. The B\&B algorithm searches the complete space of solutions for 
the best one within a given problem by branching a tree. For each iterative search, there exists an incumbent solution, which denotes to the best found feasible solution in the branching tree. If the solution is worse than the existing incumbent solution, then the branch is fathomed. The algorithm stops as soon as no subset is remained, which has to be still fathomed, and the best incumbent solution is denoted as the optimal value.

\subsubsection{Stage 2: Cooperative Secondary User Selection}

The second stage of the proposed HM2CSS scheme is to select SUs for each channel $m \in \mathcal{M}$, which together with SU-leaders cooperatively sense channels. To begin with, the received signals' envelopes for each sample $n$ are calculated as follows:

$$
r_{k}^{m}(n)=\sqrt{\left(g_{I, k}^{m}(n)\right)^{2}+\left(g_{Q, k}^{m}(n)\right)^{2}}
$$

where $g_{I, k}^{m}(n)$ and $g_{Q, k}^{m}(n)$ are the in-phase and quadrature components of the received signal by $k$-th SU sensing channel $m$, respectively. Then, the CVM test matrix is formed by calculating the received signals' envelopes correlation values and finding the difference in the correlation of the sensed signal envelopes with the sensed leaders' ones for each channel $m \in \mathcal{M}$. Thus, the CVM test matrix entries are calculated as [38]:

$$
T_{j, k}=\frac{1}{12 N}+\sum_{n=1}^{N}\left(F_{j}\left(r_{k}^{m}(n)\right)-\frac{2 n-1}{2 N}\right)^{2},
$$

where $F_{j}($.$) is probability distribution of the received signal by j$-th $\mathrm{SU}$ acting as a leader for channel $m$. It should be noted that for each channel $m$ test values have to be computed only for those SUs, which are one hop away from the leaders. Otherwise, the test value is set to 0 .

Our objective is to select SUs with the lowest correlation compared to the leader for CSS of the $m$-th channel. This implies selecting cooperative SUs with highest CVM test values as they provide lowest correlation between SUs' signal envelopes and leaders' signal envelopes. Assuming $\beta_{j k}$ to be a binary variable, which shows whether $k$-th SU is selected for CSS or not, we have: 


$$
\beta_{j k}= \begin{cases}1, & \text { if } k \text {-th } \mathrm{SU} \text { is selected as cooperative with leader } j \\ 0, & \text { otherwise }\end{cases}
$$

Then, an optimization problem for cooperative SU-selection can be formulated as:

$$
\begin{aligned}
& \max _{\beta_{j k}}: \sum_{k=1}^{K} \sum_{j=1}^{M} \beta_{j k} \times T_{j k} \times a_{j k} \\
& \text { s.t. } C 1: \sum_{j=1}^{M} \beta_{j k} \geq 1, \quad \forall k \rightarrow \text { non-leaders of each } m \in \mathcal{M} \text {, } \\
& C 2: \sum_{j=1}^{M} \beta_{j k} \geq 0, \quad \forall k \rightarrow \text { leader of each } m \in \mathcal{M}, \\
& C 3: \sum_{j=1}^{M} \beta_{j k} \leq I, \quad \forall k \rightarrow \text { non-leaders of each } m \in \mathcal{M}, \\
& C 4: \sum_{j=1}^{M} \beta_{j k} \leq I-1, \quad \forall k \rightarrow \text { leader for each } m \in \mathcal{M}, \\
& C 5: \sum_{k=1}^{K} \beta_{j k} \leq \frac{K \times I}{M}-1, \quad \forall j \rightarrow \text { leader of each } m \in \mathcal{M} \text {, } \\
& C 6: L \leq \frac{\sum_{k^{E}=1}^{K^{E}} S N R_{j k^{E}} \times \beta_{j k^{E}}}{\sum_{k^{E}=1}^{K^{E}} \beta_{j k^{E}}} \leq U, \quad \forall j \rightarrow \text { leader of each } m^{N} \in \mathcal{M}^{\mathcal{N}}, \\
& C 7: L=(1-p) \times S N R_{\text {avg }}, \quad 0 \leq p \leq 1, \\
& C 8: U=(1+p) \times S N R_{\text {avg }}, \quad 0 \leq p \leq 1,
\end{aligned}
$$

where $a_{j k}$ is the binary value 1 , if $k$-th SU can be selected as cooperative with leader $j$ and 0, otherwise, $p$ is the tolerance probability for $S N R_{\text {avg }}$, and $L$ as well as $U$ are lower and upper bounds for $S N R_{a v g}$ of all cooperative SUs of each channel $m^{N} \in \mathcal{M}^{\mathcal{N}}$, respectively.

The constraints (C1)-(C5) are introduced to balance the energy consumption among all channels and devices fairly. For each $T_{j k}$ matrix column, minimum of 1 (C1) and maximum of $I$ (C3) entries are chosen for the non-leader columns, whereas 
minimum of $0(\mathrm{C} 2)$ and maximum of $I-1(\mathrm{C} 4)$ entries are chosen for the leader ones. In order to limit the energy consumption of the system, constraint in (C5) sets the maximum number of SUs, which can sense one channel to $\frac{K \times I}{M}$. In addition to this, constraints (C6)-(C8) are introduced only for the channels, which we own no prior information about the signal being carried by them, i.e., ED-based sensed channels. It ensures fair selection of the number of ED-based SUs from lower and higher SNR values than the average SNR one with the help of lower (C7) and upper (C8) bounds for the total $S N R_{\text {avg }}$ of cooperative SUs.

The problem in (5.7) is also of MILP type with linear objective function and constraints. To solve problem (5.7), we adopted the B\&B algorithm as well. Here, the variables are of binary and integer types. In general, the $B \& B$ algorithm has the worst case complexity scenario equal to the exhausted search. However, the complexity depends on the input variables and constraints fed to the algorithm. In the best case scenario, the complexity is linear as simple parallelism and incorporation of heuristics may reduce the relaxed problem as well as the tree size [57]. The parallelism mentioned is the ability of the MILP tree nodes to be processed independently. In the formulated problem the constraint matrix is highly sparse, which reduces the complexity as well. Hence, the average complexity of the B\&B algorithm is low and maybe considered as a reasonable performance metric [57].

The problem's output can be seen as the optimized cooperative SUs' vector for each channel $m \in \mathcal{M}$ as $\mathbf{Q}=\left[\begin{array}{llll}Q_{1} & Q_{2} & \ldots & Q_{M}\end{array}\right]^{T}$, where $Q_{m}$ is the number of cooperative SUs for channel $m$, and is equal to:

$$
Q_{m}=\sum_{k=1}^{K} \beta_{j k},
$$

where $j$ is the index of a $\mathrm{SU}$, which is the leader for channel $m$.

The selected cooperative SU-clusters use diffusion-based learning, discussed in Section 4.3.3, to exchange the locally sensed information upon which it is decided if PU signals are present or not. Reaching the consensus point, the point where all cooperative SUs have the same information about the test statistics for the channel, implies performing a total of $i_{T}$ iterations. Then, the resulted test statistics are compared with the thresholds, which differ depending on the detector types, to decide if channels are occupied or not. Finally, the unoccupied channels can be assigned to SUs with the highest priority for transmitting data. 
In general, two stage optimization problems may lead to a sub-optimal solution. However, the reason why joint optimization is not considered in our case is the cooperative SU-selection dependence on leaders. Leader selection of the first stage acts as a benchmark in selecting cooperative SUs for the second stage. The second optimization problem has to be solved by leaders only instead of all SUs. Therefore, such a configuration allows complexity reduction of the system in total. Moreover, selecting correct leaders helps in an efficient selection of corresponding SUs, which results in detection performance improvements. As it will be seen in the next section, the two stage solution in our case is proved to have stable system performance and outperform the existing multi-band CSS schemes.

\subsection{Simulation Results}

In this section, the performance of the proposed HM2CSS scheme is investigated. Three different network sizes are considered for simulation results. Those are $M=50$ channels and $K=100 \mathrm{SUs}, M=25$ channels and $K=50 \mathrm{SUs}$, and $M=5$ channels and $K=10$ SUs. On the event that only one network size is used, the $M=50$ channels and $K=100$ SUs scenario is assumed. The information available about channels is divided according to Table 5.1, such that for each case of $M$ there are $M^{N}$ channels with no prior information about PU signals, $M^{P}$ channels with known pilot tones of PU signals, and $M^{O}$ channels with OFDM signals being sent over them by PUs. No prior information PU signals are modeled as random Gaussian, as it has been discussed in Chapter 2. PU signals with known pilot tones are assumed to have binary phase shift keying (BPSK) modulated pilot tones and random Gaussian

Table 5.1: Number of channels with the certain information available about them for the total of $M$ channels.

\begin{tabular}{|c||c|c|c|}
\hline$M$ & $M^{N}$ & $M^{P}$ & $M^{O}$ \\
\hline \hline 50 & 20 & 20 & 10 \\
\hline 25 & 10 & 10 & 5 \\
\hline 5 & 2 & 2 & 1 \\
\hline
\end{tabular}


data-carrying signal parts, where $10 \%$ of the total PU signal power is allocated for the pilot tone. As for OFDM PU signals, 16 QAM modulation is used. In total, 100 blocks are used to construct one OFDM signal, where the number of symbols is assumed to be 32 with the cyclic prefix length of 8 for each block. In all cases, the amount of ED, PD, and OD-based SUs are assigned to be approximately $40 \%$, $40 \%$, and $20 \%$, respectively. In an IoT-based CRN, 100 secondary IoT-nodes is a reasonable number due to the fact that many sensor nodes can connect with each one of them. For instance, assuming 10 sensors nodes per house, each network represents by itself 4000 sensor nodes in smart homes applications [58]. All parameters set to the network model are as follows, unless specified. The minimum and maximum amount of channels sensed by one $\mathrm{SU}$ is set to be 1 and $I=5$, respectively. $S N R_{m k}$ is chosen randomly, with the $S N R_{\text {avg }}$ value of $-10 \mathrm{~dB}$ for each channel. In addition to this, a fixed number of samples $N_{m}$ is used by SUs while sensing each channel. This is done for synchronization purposes of the diffusion-based distributed learning. The number of samples sensed by one ED, PD, and OD-based SU per channel are considered $N_{E D}=2000, N_{P D}=500$, and $N_{O D}=4000$, respectively. As for the spectrum sensing energy consumption, $40 \mathrm{~nJ}$ of energy is assumed to be consumed by an $\mathrm{SU}$ per sensing one sample [59]. The IEEE 802.22 standard is taken into account when performing simulation results, according to which the total probability of detection should be greater than $90 \%$, while the false alarm probability should be less than $10 \%$.

Simulation results are illustrated in terms of diffusion learning, receiver operating characteristics (ROC), aggregate PU protection level vs $S N R_{\text {avg, }}$, aggregate CRN throughput, and energy consumption level on the sensing process. The results are compared to the existing distributed and centralized schemes. RSSS is chosen as a benchmark for the existing distributed scheme due to its simplicity and low computational complexity favored by IoT devices. In RSSS, cooperative SUs are chosen in an arbitrary manner, i.e., randomly $[6,36]$. As for the existing centralized schemes, OR, k-out-of-K, majority, and AND rules are chosen to be compared to the proposed distributed HM2CSS scheme [6]. The k-out-of-K rule considered assumes that for channels to be assumed busy, minimum $20 \%$ of SUs sensing channels detect the presence of PUs. The following sub-sections provide a thorough explanation and comparison of the achieved results. 


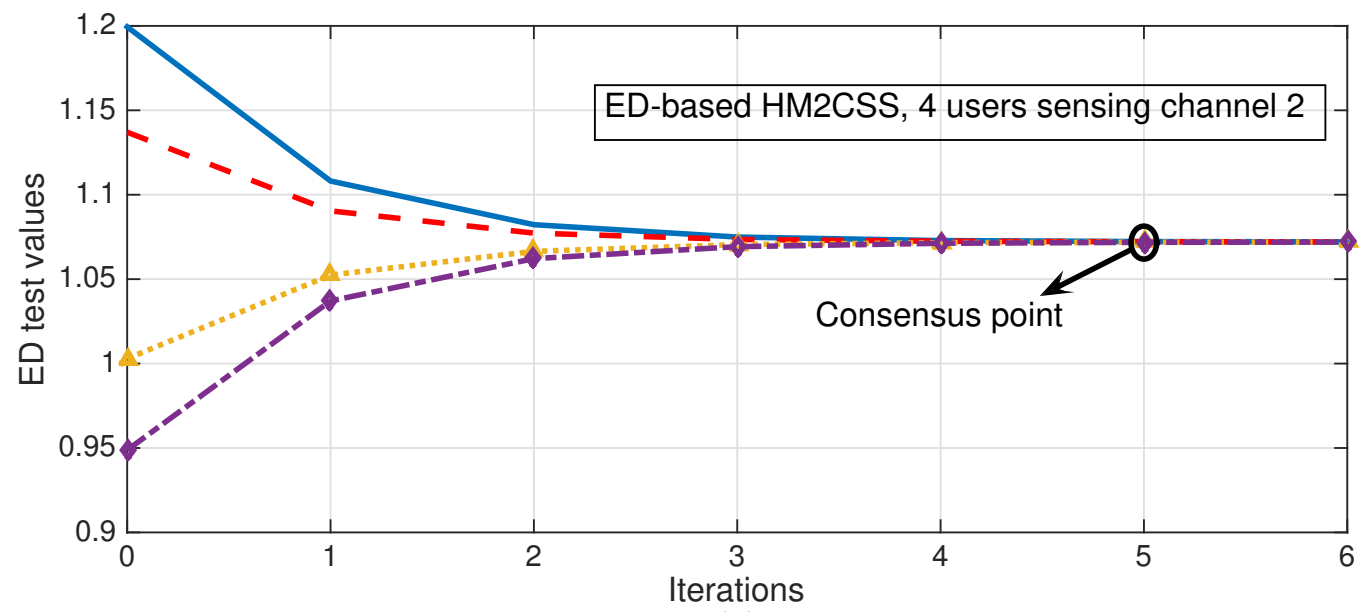

(a)

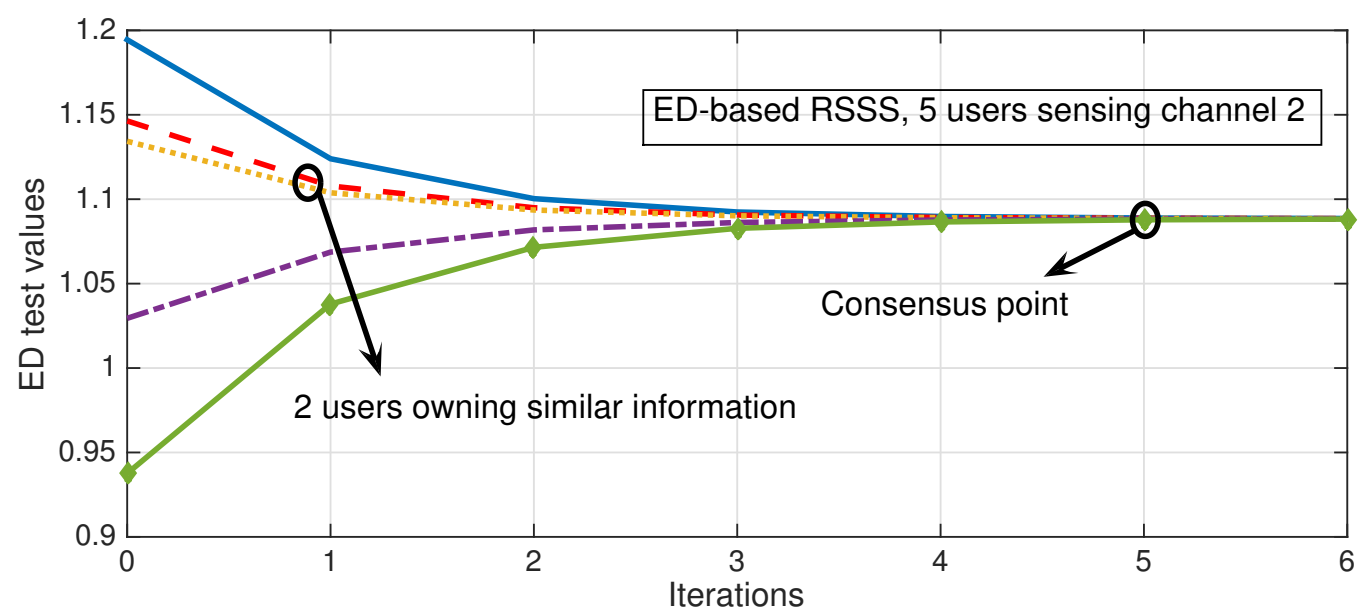

(b)

Figure 5.3: The diffusion learning process illustration of the ED-based channel 2 for: (a) the proposed HM2CSS scheme and (b) the existing RSSS scheme.

\subsubsection{Diffusion Learning Analysis}

Fig. 5.3 illustrates the learning process of (a) the proposed HM2CSS scheme used to select cooperative ED-based SUs in order to sense channel 2 and (b) the existing RSSS scheme used to select cooperative ED-based SUs in order to sense channel 2. Fig. 5.4 


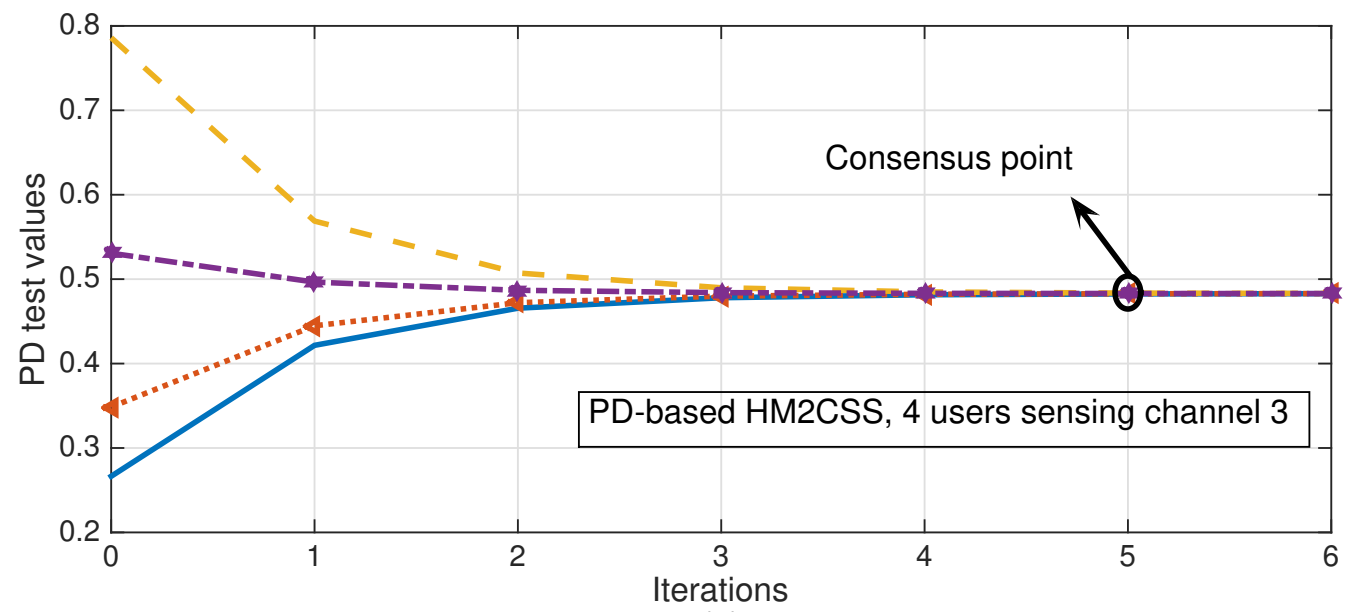

(a)

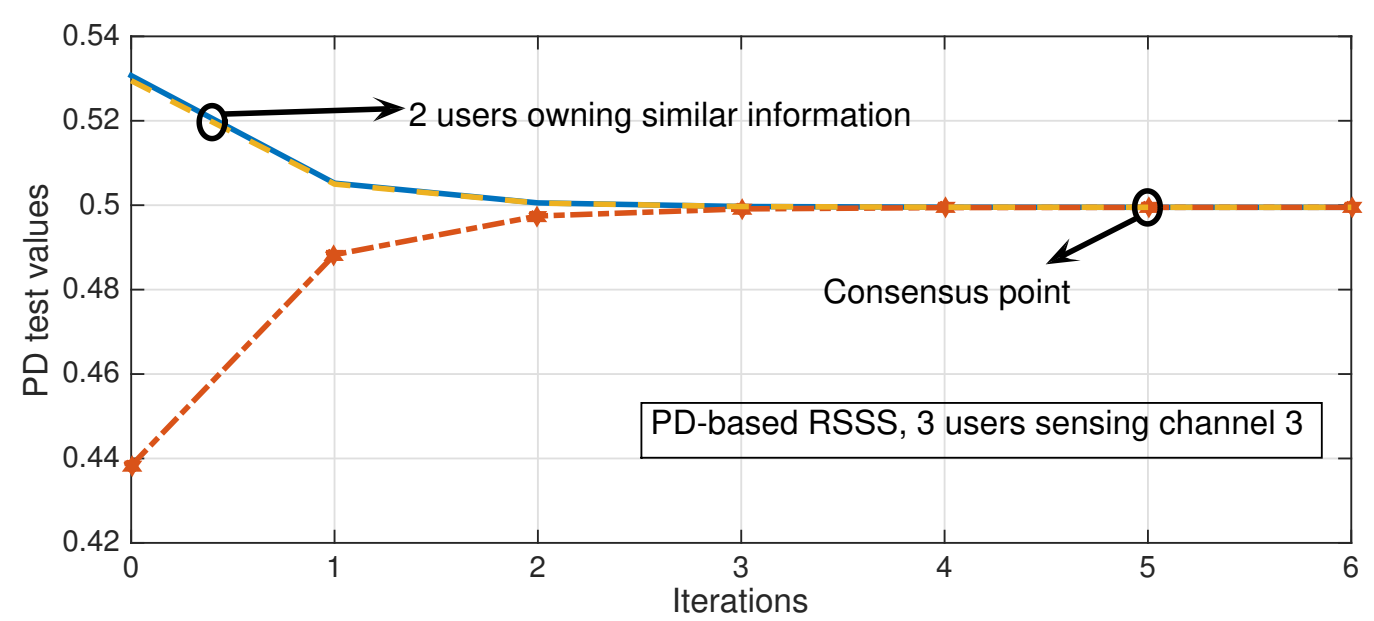

(b)

Figure 5.4: The diffusion learning process illustration of the PD-based channel 3 for: (a) the proposed HM2CSS scheme and (b) the existing RSSS scheme.

shows the learning process of (a) the proposed HM2CSS scheme used to select cooperative PD-based SUs in order to sense channel 3 and (b) the existing RSSS scheme used to select cooperative PD-based SUs in order to sense channel 3. In addition, Fig. 5.5 demonstrates the learning process of (a) the proposed HM2CSS scheme used to select cooperative OD-based SUs in order to sense channel 5 and (b) the existing RSSS scheme used to select cooperative OD-based SUs in order to sense channel 5. In all three cases it can be observed that one of the main advantages of the proposed 


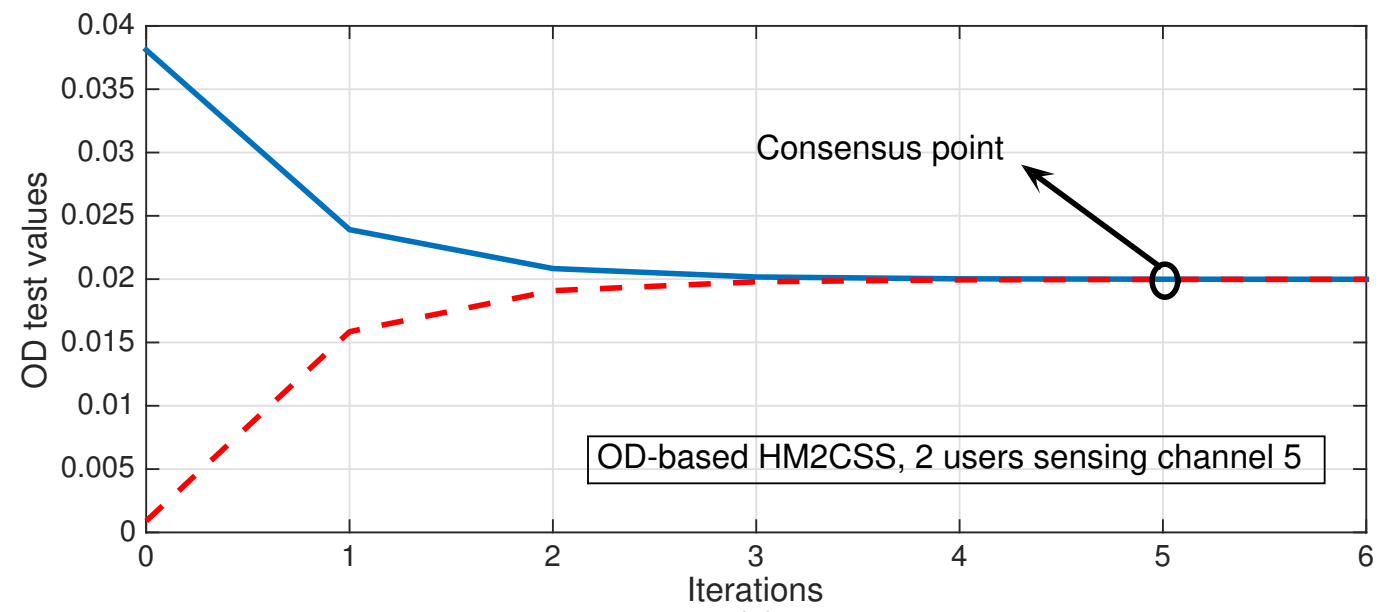

(a)

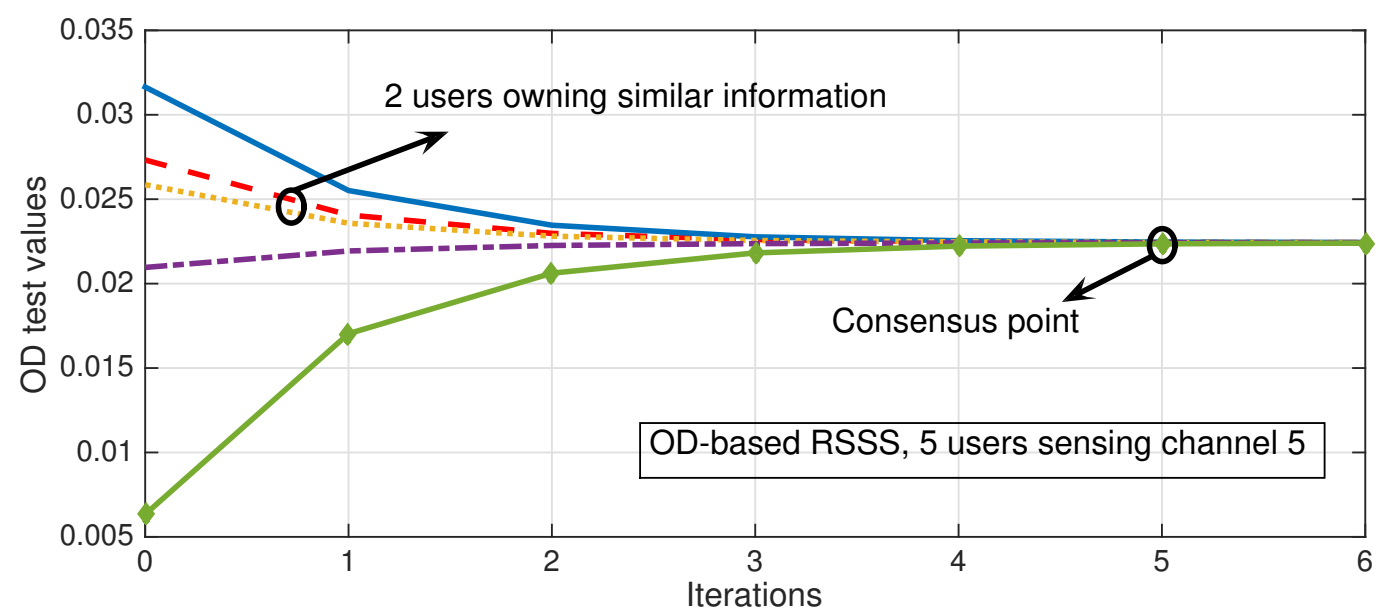

(b)

Figure 5.5: The diffusion learning process illustration of the OD-based channel 5 for: (c) the proposed HM2CSS scheme and (d) the existing RSSS scheme.

HM2CSS scheme is that it does not allow the choice of redundant cooperative SUs, the ones with similar information sensed for the $m$-th channel. However, this does not mean that the number of chosen cooperative SUs to sense channels decreases. For the proposed HM2CSS scheme it depends on the correlation of received signal envelopes, while for the existing RSSS scheme it is random. This means that the environment and channel conditions may affect the choice for the proposed HM2CSS scheme. Nevertheless, the number of cooperative SUs is restricted by the constraints, 
so in both cases this number cannot exceed the limits of $\frac{K \times I}{M}$ for one channel. For the proposed HM2CSS scheme this limitation can be seen in (5.7). After 5 iterations, the consensus point is reached and results are compared to the threshold so that the occupancy of each channel is determined.

\subsubsection{Receiver Operating Characteristic Results}

This section represents ROC characteristics for the proposed HM2CSS scheme from different prospectives. Fig. 5.6 compares ROC for the proposed HM2CSS scheme

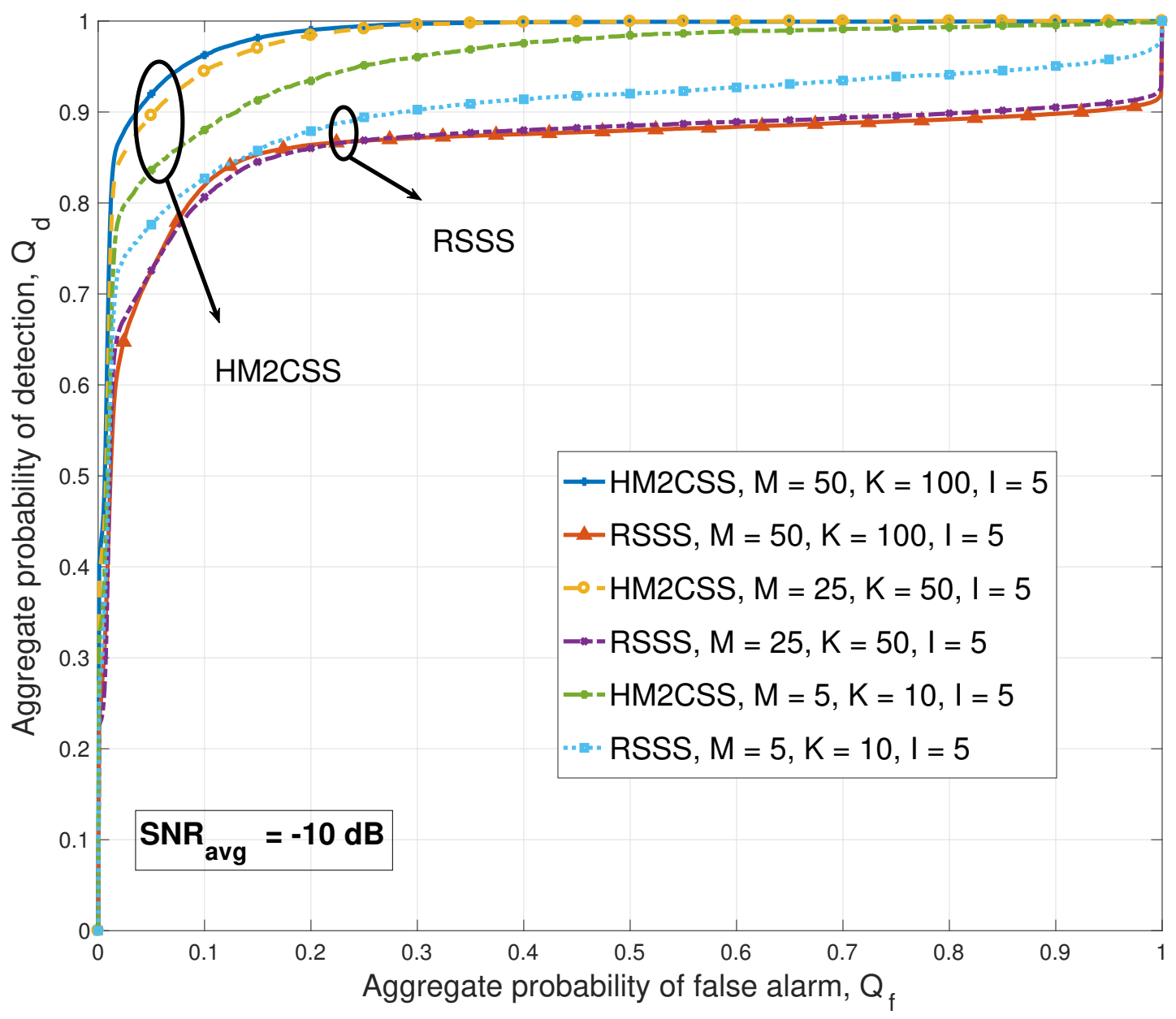

Figure 5.6: Global ROC results for the proposed distributed HM2CSS scheme compared to the existing distributed RSSS scheme for different network sizes. 
with the existing RSSS scheme. Several important facts can be noted from the curves illustrated. To begin with, the proposed HM2CSS scheme tends to outperform RSSS in all three cases. It fulfills the global detection probability requirements of $Q_{d}=90 \%$ for the global false alarm probability of $Q_{f} \leq 10 \%$ in cases of $M=50$ channels and $K=100 \mathrm{SUs}$ as well as $M=25$ channels and $K=50 \mathrm{SUs}$. Whereas for the case of $M=5$ channels and $K=10$ SUs, the global probability of detection $Q_{d}=90 \%$ is reached for the global probability of false alarm $Q_{f}$ of a bit higher than $10 \%$. Moreover, it can be noticed that as soon as the network size increases, the performance of the proposed HM2CSS scheme improves. It is caused by a wider variety in selection

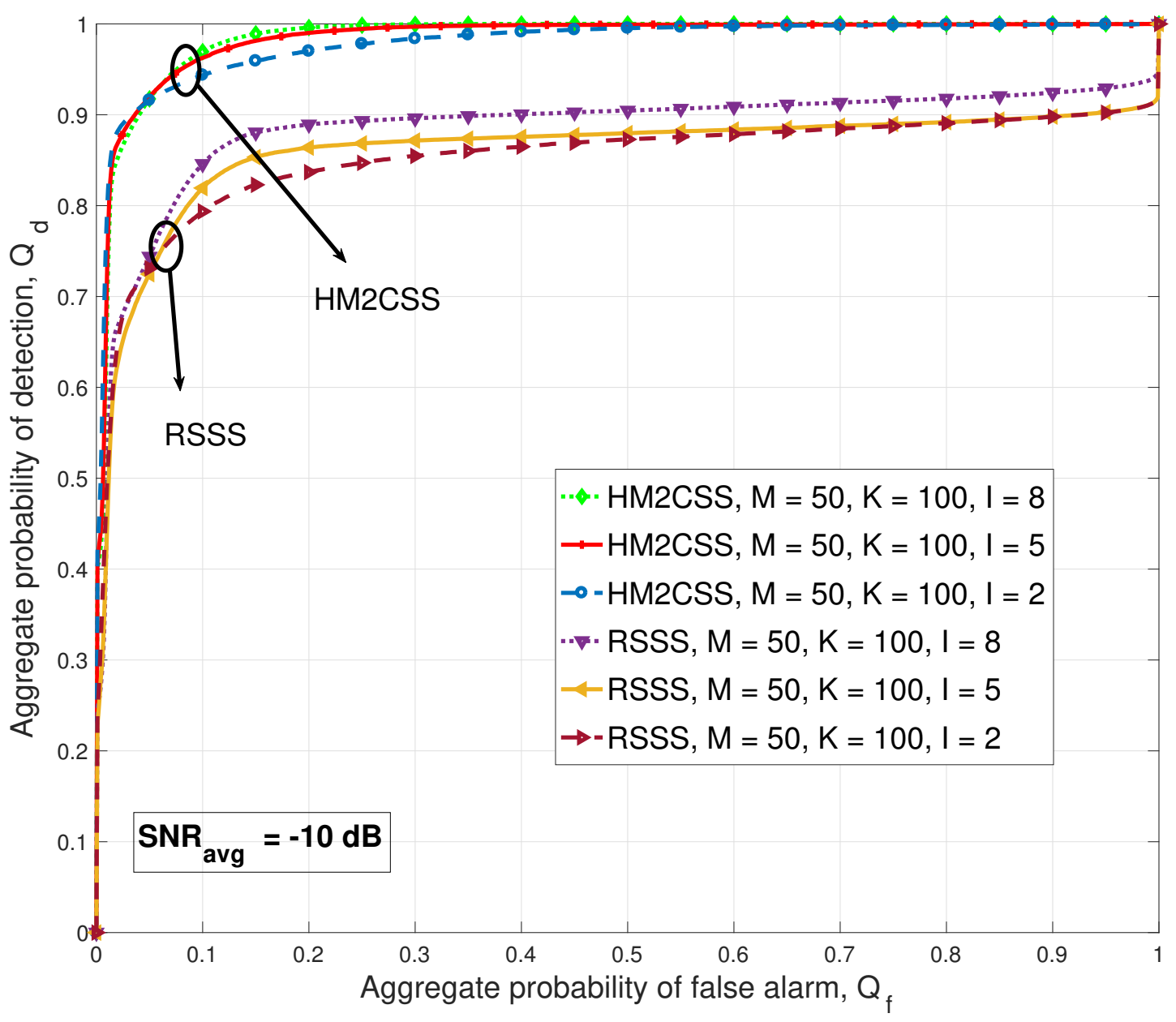

Figure 5.7: Global ROC results with various maximum number of channels allowed to be sensed by one SU for both the proposed distributed HM2CSS scheme and the existing distributed RSSS scheme. 
of cooperative SUs. This makes the system applicable to the future IoT networks by introducing the scalability advantage. However, increasing the network size decreases the system performance for the existing RSSS scheme. This can be explained by the way the RSSS scheme is accomplished or, in other words, the way cooperative SUs are being chosen. Due to the fact that in RSSS cooperative SUs are chosen in a random manner, the chances to get cooperative SUs with at least one of them to own feasible information for the channel decreases as soon as the network starts increasing in size. As a result, this leads to system performance degradation.

Fig. 5.7 discovers how changing the maximum number of channels sensed by one

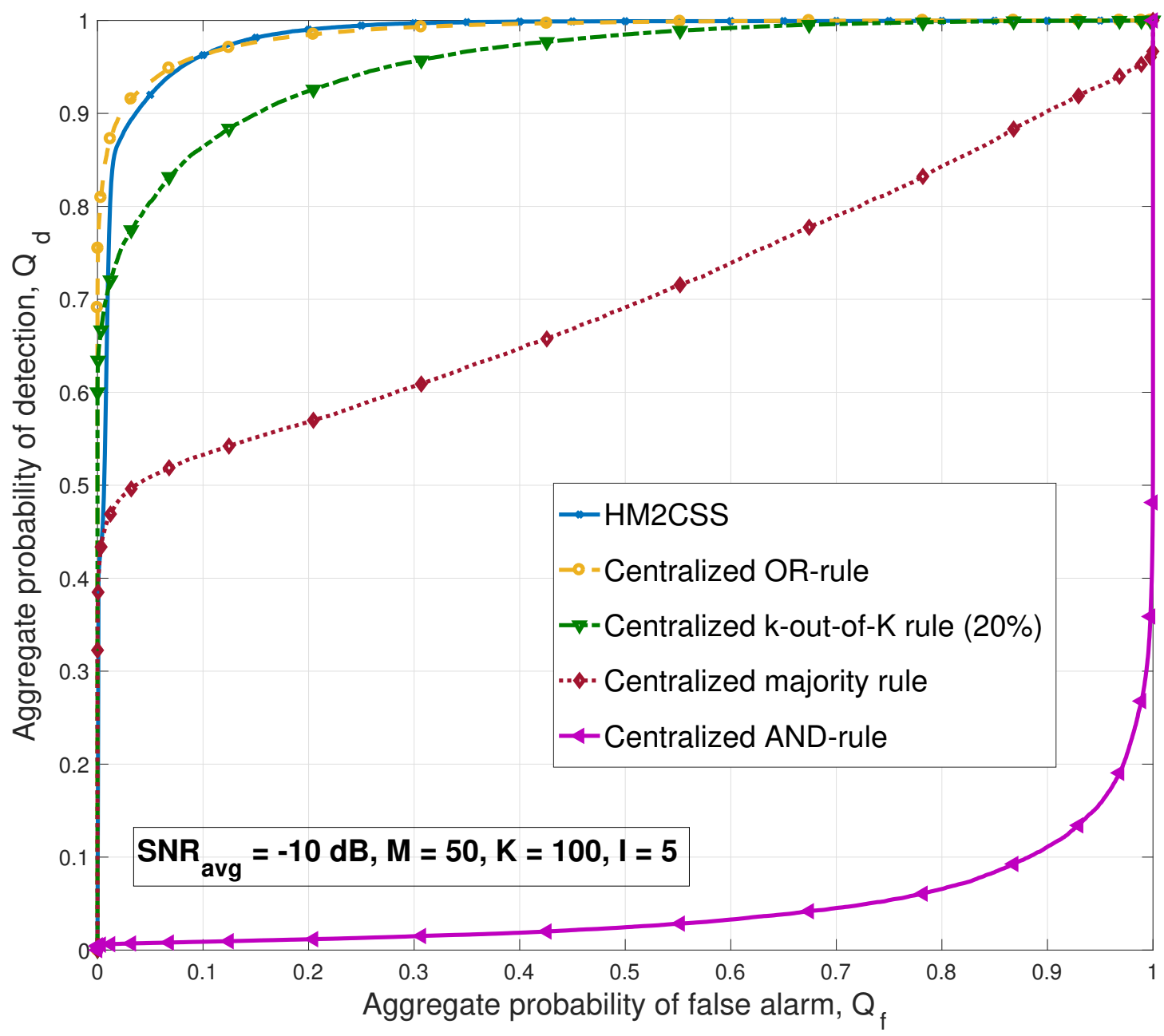

Figure 5.8: Global ROC results for the proposed distributed HM2CSS scheme compared to the existing centralized schemes. 
SU affects the aggregate ROC performance of both, the proposed HM2CSS and the existing RSSS schemes. It can be seen that in case of the proposed HM2CSS scheme, the enhancement of ROC results stops at a certain point of increasing $I$. This allows us to have decreased energy consumption per SU as sensing of additional channels requires extra power to be consumed on both sensing and transmission processes. It can be seen that the aggregate detection probability for the cases of $I=2$ and $I=5$ is slightly higher than the one for $I=8$ in case the aggregate false alarm probability is low, i.e., $Q_{f} \leq 5 \%$. This is due to the fact that the selection of cooperative SUs for the proposed HM2CSS scheme is being optimized. Choosing best 5 channels to sense by each SU can provide us with the better performance than sensing higher numbers of channels as 8 in case some of them are in the deep fading state with respect to SUs. This places another advantage for low-power IoT devices, which are not capable of sensing more than a certain number of channels at a time instance. However, for the existing RSSS technique, the system performance keeps growing up by increasing the number of channels sensed by one SU. The more randomly selected cooperative SUs are selected to sense channels, the better the system performance is. Randomness in the process can not guarantee that the selected channels to be sensed by a certain SU are not in deep fade state with respect to the SU. This is why choosing more channels to sense can enhance the performance in this case. It is a certain disadvantage of the RSSS process as higher amount of energy is needed to sense greater number of channels, which can create power consumption problems for IoT systems.

While Figs. 5.6 and 5.7 illustrate the proposed distributed HM2CSS scheme with another existing distributed scheme, Fig. 5.8 compares HM2CSS with the existing centralized techniques. Generally, it is a well known fact that the centralized schemes of OR and k-out-of-K rules tend to outperform distributed schemes in terms of ROC because of the high control level accommodated by an FC [60]. However, Fig. 5.8 illustrates that the proposed scheme outperforms most of the centralized rules as k-out-of-k, majority, and AND-rule and provides the performance of approximately the centralized OR-rule one. Both, the proposed distributed HM2CSS as well as centralized OR-rule satisfy the global probability of detection $Q_{d}=90 \%$ at the global probability of false alarm $Q_{f}$ of less than $10 \%$.

Finally, Fig. 5.9 shows ROC results for the proposed HM2CSS scheme for both 


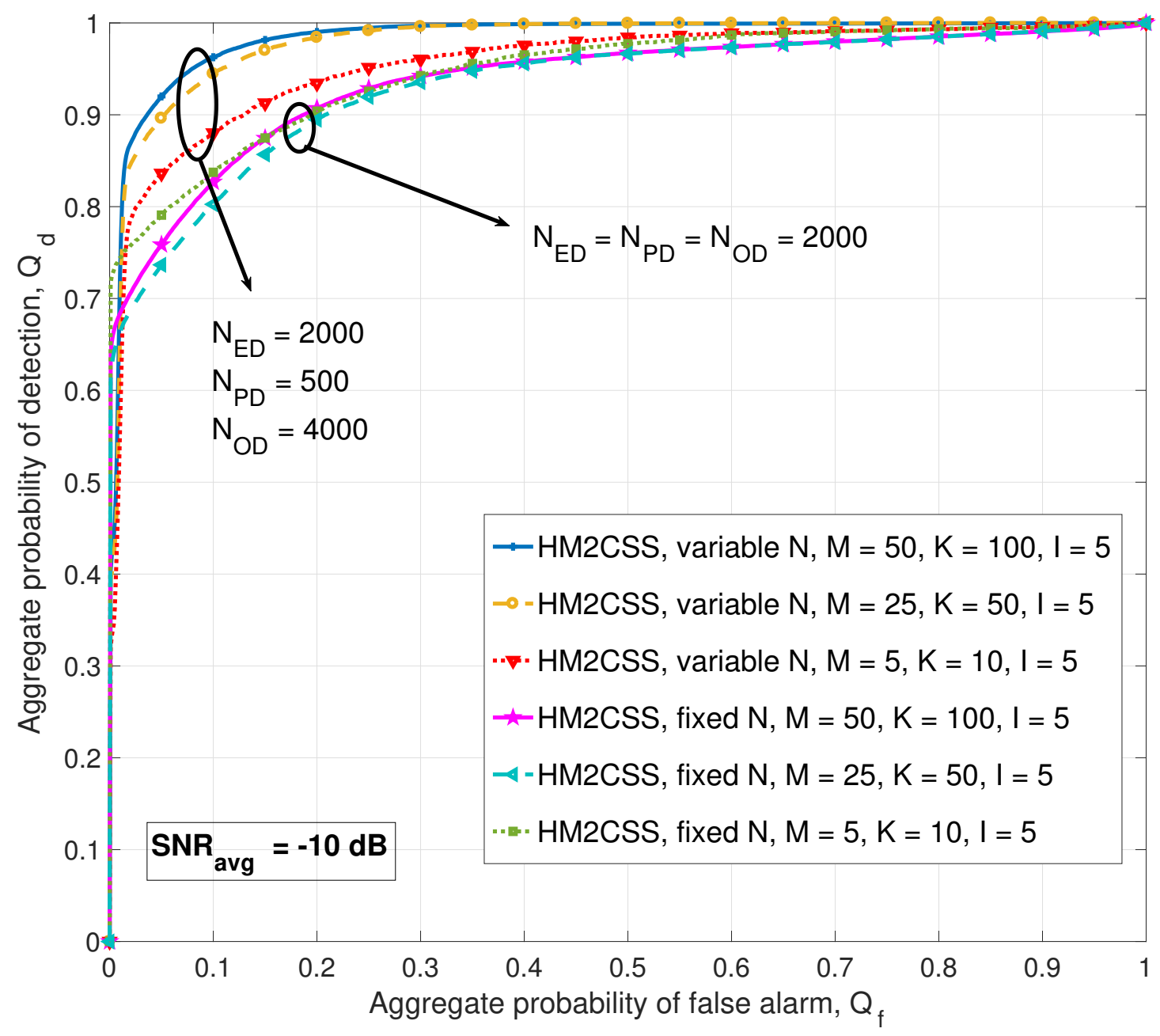

Figure 5.9: Global ROC results for the fixed and variable number of samples between cooperative SU-clusters of the proposed distributed HM2CSS scheme.

variable and fixed amount of samples being sensed by each SU for each channel. Synchronization during the learning process is an important issue for the distributed systems [6]. However, the synchronization issue comes within cooperative SUs. Hence, different clusters of cooperative SUs may sense channels using different number of samples per channel. This represents an advantage to heterogeneous systems in general as the number of samples needed for different types of detectors may vary. ROC with fixed $N_{E D}=N_{P D}=N_{O D}=2000$ results are compared with the allowed variation in the number of samples per channel as $N_{E D}=2000, N_{P D}=500$, and $N_{O D}=4000$. In the results of Fig. 5.9 it can be observed that the allowed variation 
in samples sensed by each detector type proves to enhance the ROC performance without disturbing synchronization within cooperative SUs' learning as only similar detector types may be chosen in the same learning clusters.

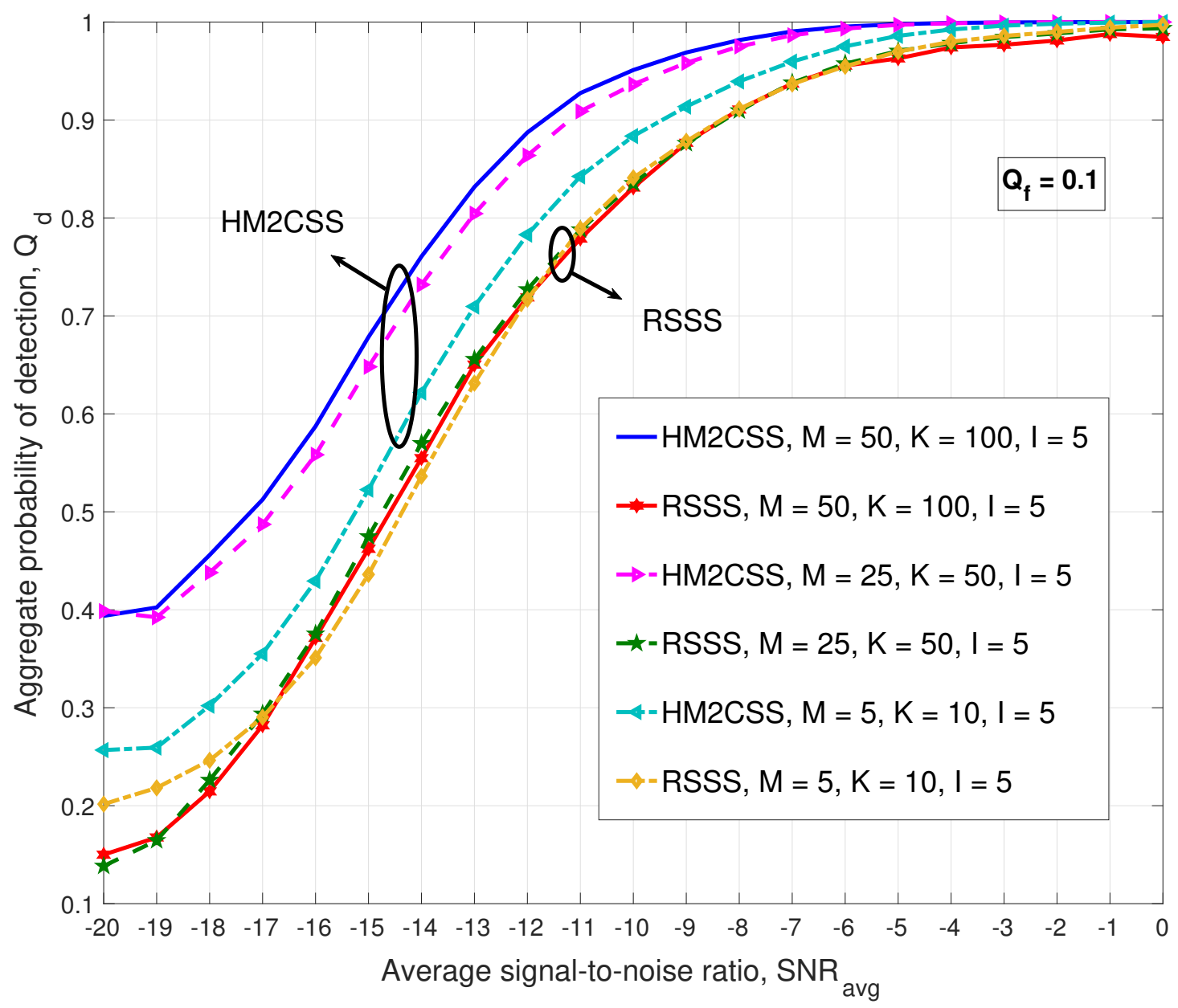

Figure 5.10: Global PU protection level vs $S N R_{\text {avg }}$ results for the proposed distributed HM2CSS scheme compared to the existing distributed RSSS scheme for different network sizes. 


\subsubsection{Aggregate Primary User Protection Level vs Average Signal-to-Noise Ration Analysis}

PU protection level or global probability of detection, $Q_{d}$, with respect to different values of $S N R_{\text {avg }}$ for the proposed HM2CSS scheme is compared to the existing RSSS scheme in Fig. 5.10. In this case, the probability of false-alarm is fixed and set to $10 \%$. It is clear that for all $S N R_{\text {avg }}$ values, the proposed HM2CSS scheme outperforms the existing RSSS scheme. The global probability of detection reaches

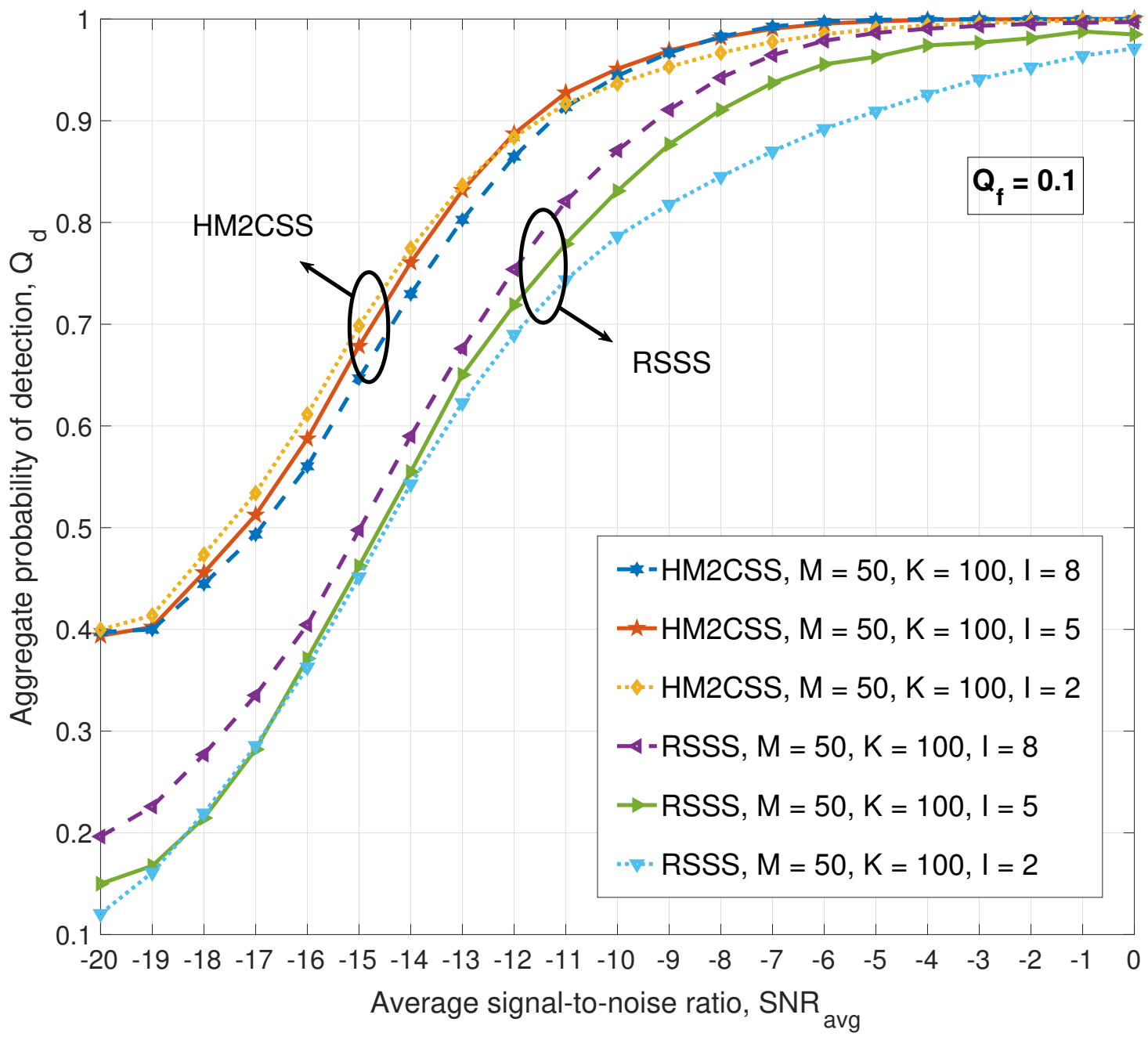

Figure 5.11: Global PU protection level vs $S N R_{\text {avg }}$ results for different values of maximum number of channels sensed by one SU for the proposed distributed HM2CSS scheme and the existing distributed RSSS scheme. 
$90 \%$ for the proposed HM2CSS scheme at $S N R_{\text {avg }}$ of slightly higher than $-12 \mathrm{~dB}$ for $M=50$ channels and $K=100 \mathrm{SUs},-12 \mathrm{~dB}$ for $M=25$ channels and $K=50 \mathrm{SUs}$, and $-9 \mathrm{~dB}$ for $M=5$ channels and $K=10$ SUs cases. Whereas, in all three cases the RSSS scheme achieves the minimum required global probability of detection at $S N R_{a v g}=-8 \mathrm{~dB}$. It can be concluded that at the $S N R_{a v g}$ value of $-10 \mathrm{~dB}$, the proposed HM2CSS scheme outperforms RSSS by $14.5 \%, 12 \%$, and $7.2 \%$ for the cases of $M=50$ channels and $K=100 \mathrm{SUs}, M=25$ channels and $K=50 \mathrm{SUs}$, and $M=5$ channels and $K=10$ SUs, respectively.

Fig. 5.11 discloses the effect of the maximum number of channels $I$ sensed by one SU by changing $S N R_{\text {avg }}$ for the proposed HM2CSS and existing RSSS schemes. The values of $I=8, I=5$, and $I=2$ are considered for simulation results. In case of the proposed HM2CSS scheme, increasing $I$ does not have a drastic impact on results. For all listed values of $I$, the global probability of detection reaches $90 \%$ at the $S N R_{\text {avg }}$ values under $-11 \mathrm{~dB}$. Moreover, at the $S N R_{\text {avg }}$ value of $-10 \mathrm{~dB}$, all three cases considered have the global detection probability of approximately $95 \%$. However, in the case of RSSS, the increase in the global probability of detection makes difference. For the given $I$ values of 8,5 , and 2, the corresponding global probability of detection reaches $90 \%$ at the values of $-9 \mathrm{~dB},-8 \mathrm{~dB}$, and $-6 \mathrm{~dB}$, respectively. This means that at the $S N R_{a v g}$ value of $-10 \mathrm{~dB}$, the IEEE 802.22 standard requirement of $Q_{d} \geq 90 \%$ is not reached. The global probability of detection at the $S N R_{a v g}$ value of $-10 \mathrm{~dB}$ is $86 \%, 83 \%$, and $78 \%$ for the $I$ values of 8,5 , and 2 , respectively. This brings an advantage of HM2CSS as in case a SU is not capable of sensing $I$ channels due to power limitations and has to decrease its value, the system performance will not experience sensible changes.

Fig. 5.12 takes into account the comparison between the proposed distributed HM2CSS scheme and the existing centralized schemes discussed above. Although at very low values of $S N R_{\text {avg }}$, centralized OR and k-out-of-K rules tend to outperform the proposed HM2CSS scheme, already at $S N R_{\text {avg }}$ of slightly higher than $-17 \mathrm{~dB}$ and $-14 \mathrm{~dB}$, the proposed HM2CSS scheme outperforms the k-out-of-K and OR centralized rules, respectively. This can be explained as follows. At very low SNR values noise level is high, and the centralized system tends to outperform due to high control level provided by the FC. However, at the $S N R_{a v g}$ value of $-10 \mathrm{~dB}$, the proposed distributed HM2CSS scheme outperforms the centralized OR, k-out-of-K, 


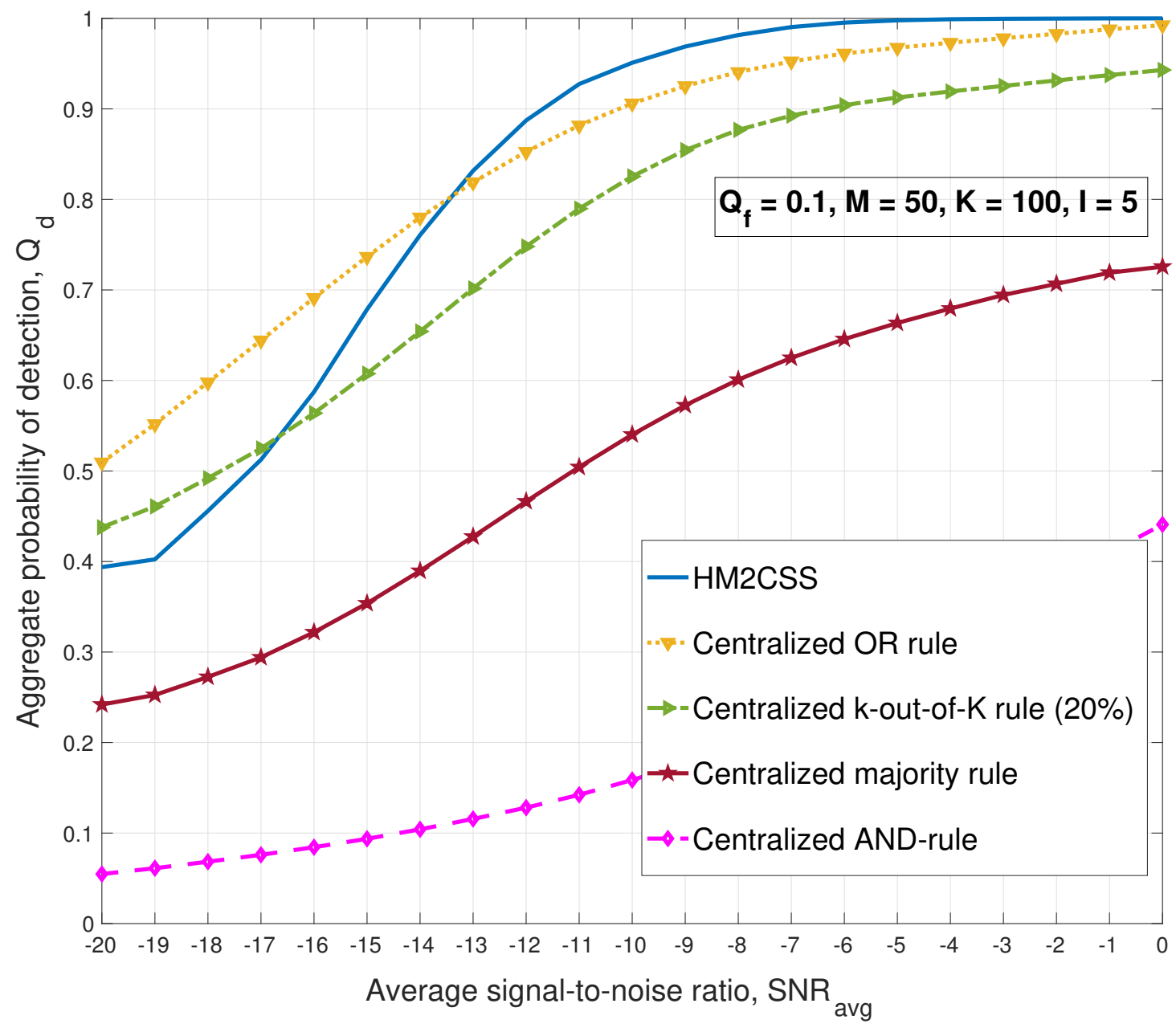

Figure 5.12: Global PU protection level vs $S N R_{\text {avg }}$ results for the proposed distributed HM2CSS scheme compared to the existing centralized schemes.

majority, and AND rules by $5.6 \%, 15.9 \%, 75.9 \%$, and more than 5 times, respectively, with the aggregate probability of detection value of $95 \%$.

Fig. 5.13 illustrates curves when the global probability of false alarm values are $Q_{f}=10 \%$ and $Q_{f}=30 \%$. The enhancement for the proposed HM2CSS scheme is in the similar manner for all three network size cases when increasing the values of global false alarm probability. Moreover, this enhancement has similar trend of approximately $10 \%$ in the aggregate probability of detection values for the $S N R_{\text {avg }}$ value of $-10 \mathrm{~dB}$. Finally, the increase in the system performance can be noticed as the network size grows. 


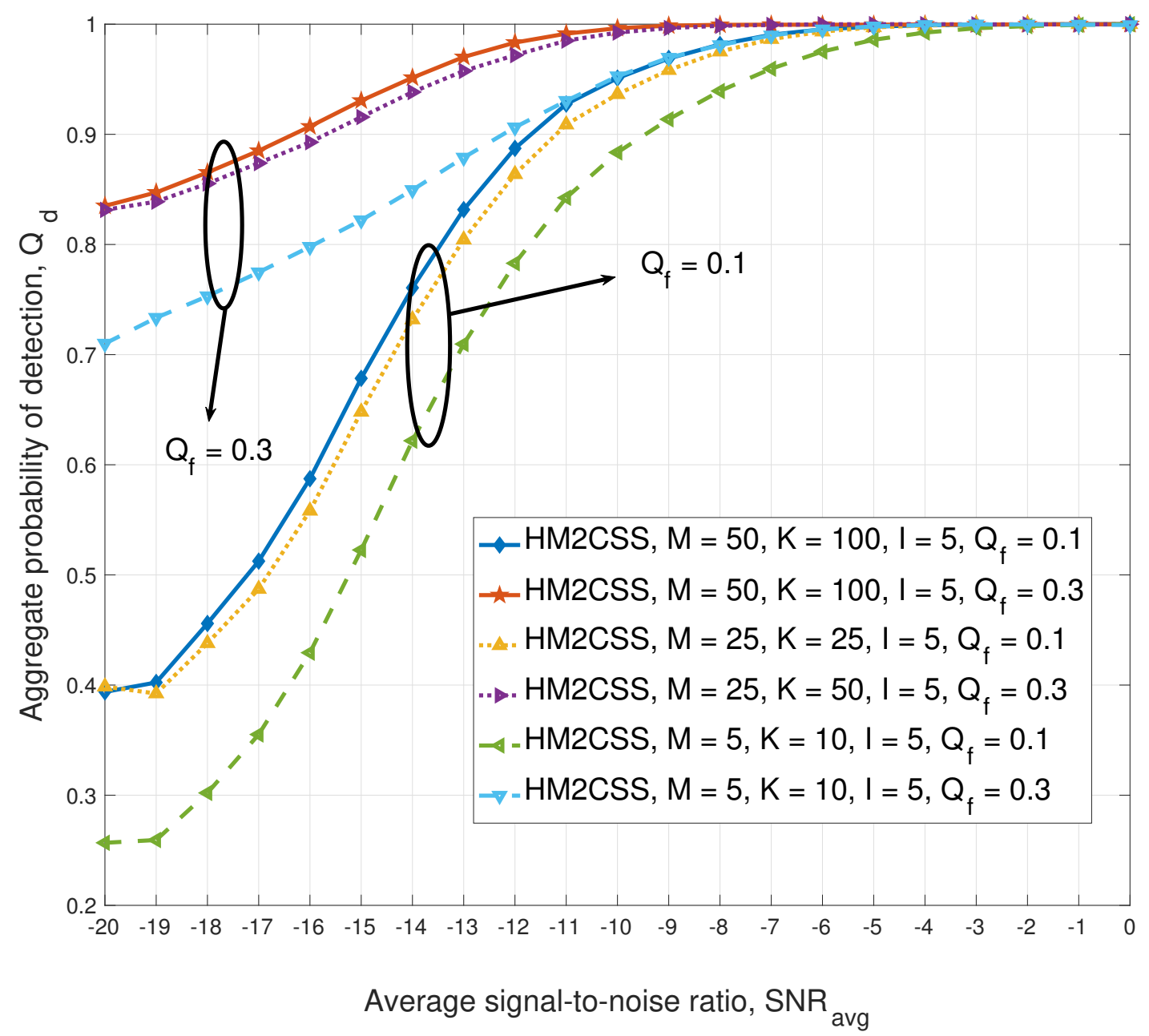

Figure 5.13: Global PU protection level vs $S N R_{\text {avg }}$ results for different values of global false alarm probability being fixed for the proposed HM2CSS scheme.

\subsubsection{Throughput Analysis}

In this sub-section, CRN throughput results of the proposed distributed HM2CSS scheme are compared with the existing distributed and centralized schemes. Concerning the IEEE 802.22 standard mentioned above, the aggregate false alarm probability is set to be $10 \%$. The interference level coming from each PU is set to be $-10 \mathrm{~dB}$, while the channel bandwidth as well as the activity of PUs is set to be $6 \mathrm{MHz}$ and $30 \%$, respectively. All simulation results are performed with respect to the aggregate PU protection level or the global probability of detection. 
Fig. 5.14 compares the proposed distributed HM2CSS scheme with the existing distributed RSSS scheme. It is clear that the proposed HM2CSS scheme tends to outperform the existing RSSS scheme. Already at the global detection probability of $60 \%, 65 \%$, and $87 \%$, the results of the existing RSSS scheme start to degrade with respect to the proposed HM2CSS scheme for the cases of $M=50$ channels and $K=100$ SUs, $M=25$ channels and $K=50$ SUs, and $M=5$ channels and $K=10$ SUs, respectively. It can also be noted that for the existing RSSS scheme, the aggregate throughput drops suddenly to very low values at the global detection probability of approximately $90 \%$ for all three networks mentioned above. However, the proposed HM2CSS scheme maintains the smooth logarithmic curve achieving better throughput results by 7.3, 3.6, and 1.4 times for the cases of $M=50$ channels

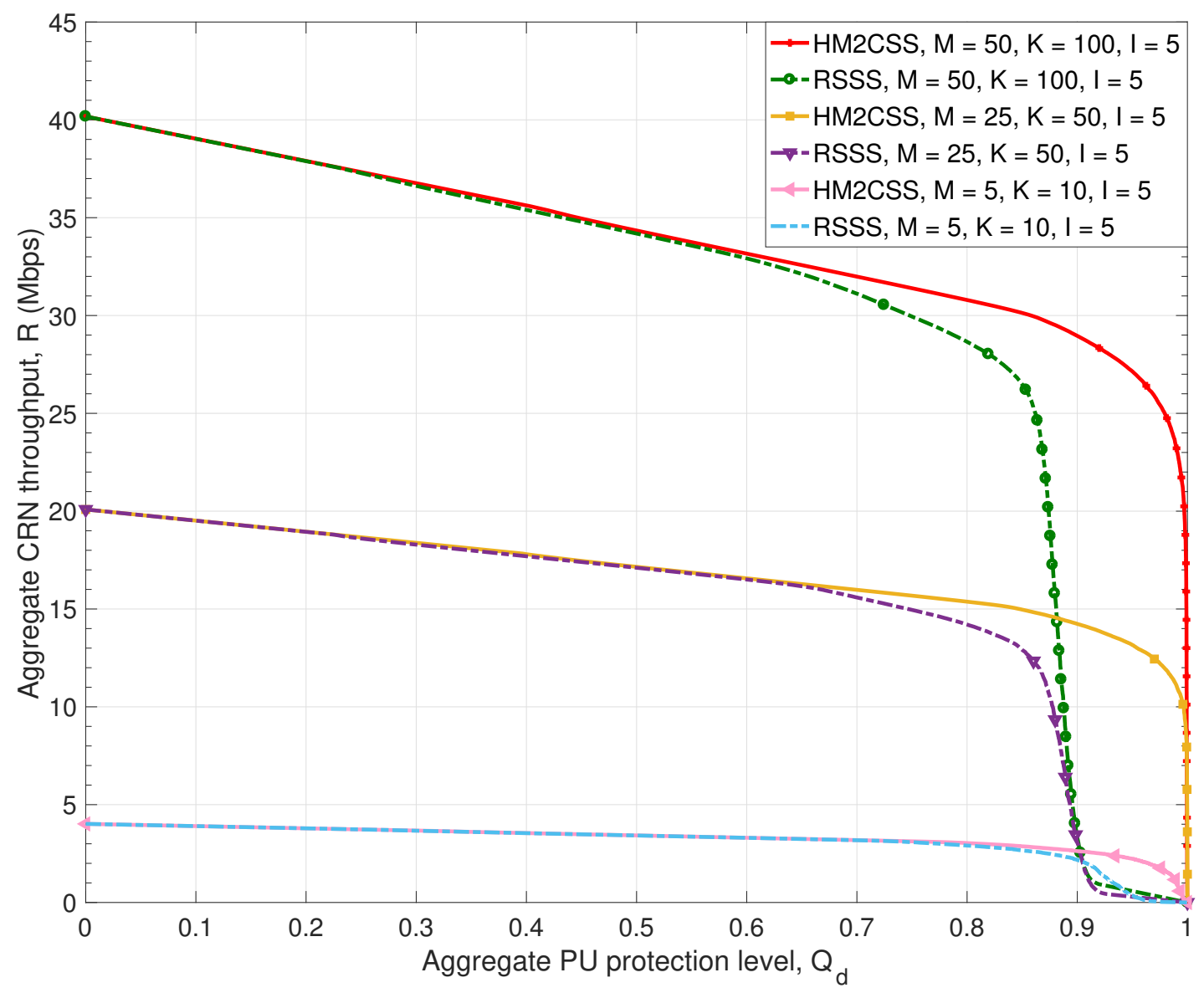

Figure 5.14: Aggregate CRN throughput results for the proposed distributed HM2CSS scheme and the existing distributed RSSS scheme. 
and $K=100$ SUs, $M=25$ channels and $K=50$ SUs, and $M=5$ channels and $K=10$ SUs, respectively. The enhancement of the proposed HM2CSS scheme significantly grows up with respect to the existing RSSS scheme as the network size grows.

Fig. 5.15 illustrates the comparison between the proposed distributed HM2CSS scheme and the existing centralized schemes. HM2CSS tends to perform in a similar manner as the centralized OR rule, achieving 29 Mbps of aggregate CRN throughput for the global detection probability of approximately 90\%. Moreover, HM2CSS outperforms the $20 \%$ k-out-of-k, majority, and AND rules by $11.5 \%, 7.25$ times, and 19.3 times, respectively.

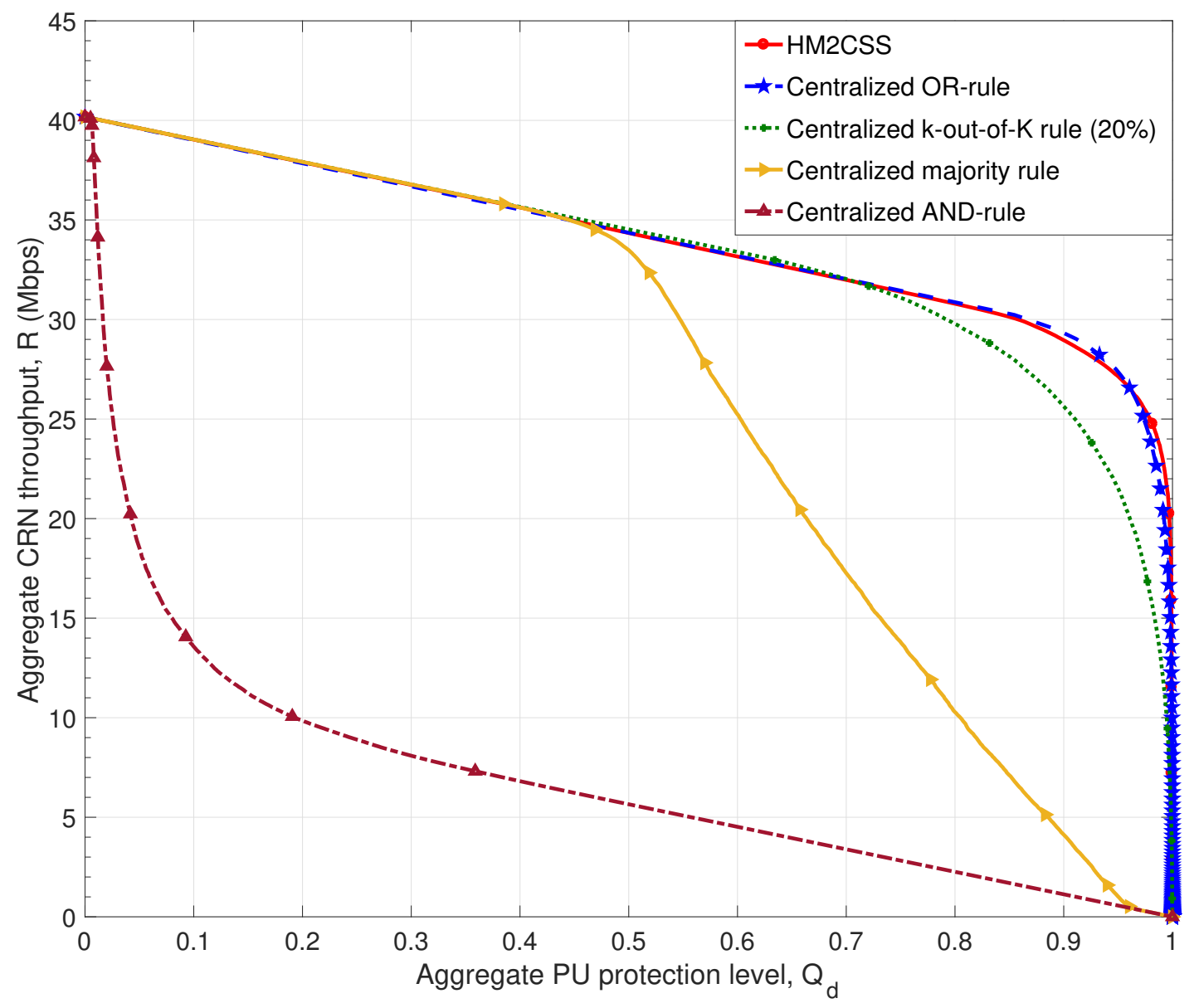

Figure 5.15: Aggregate CRN throughput results for the proposed distributed HM2CSS scheme and the existing centralized schemes. 

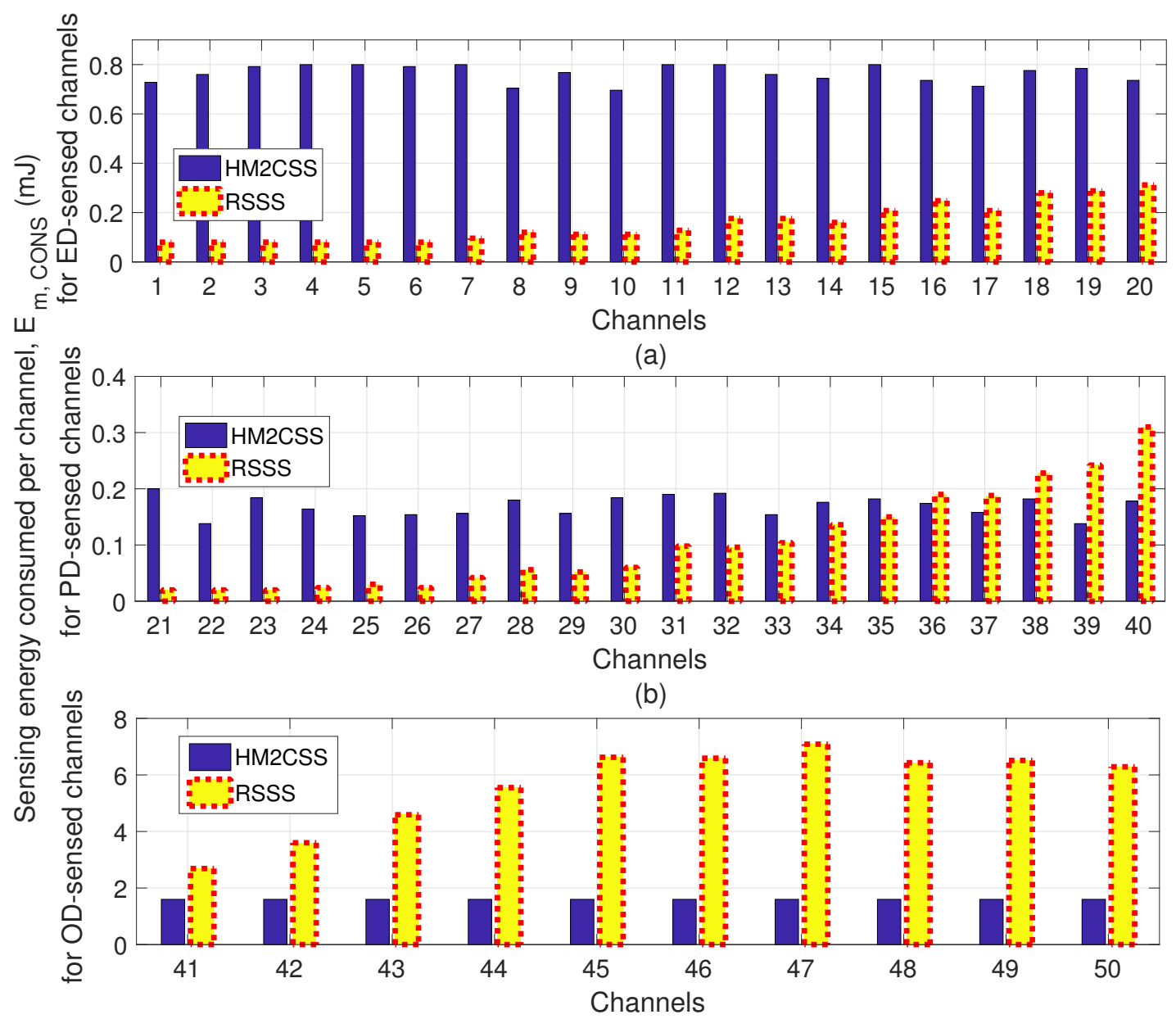

(c)

Figure 5.16: Comparison of the proposed HM2CSS scheme with the existing RSSS scheme in terms of average energy consumption level across each: (a) ED-sensed channel, (b) PD-sensed channel, and (c) OD-sensed channel.

\subsubsection{Energy Consumption on the Sensing Process}

Figs. 5.16, 5.17, and 5.18 illustrate average energy consumption on spectrum sensing. The results of the proposed HM2CSS scheme are compared with the existing RSSS scheme.

Fig. 5.16 shows average energy consumption on spectrum sensing for different channels. The proposed HM2CSS scheme fairly distributes energy consumption on the sensing process for all channels of the same type, while the existing multi-band 


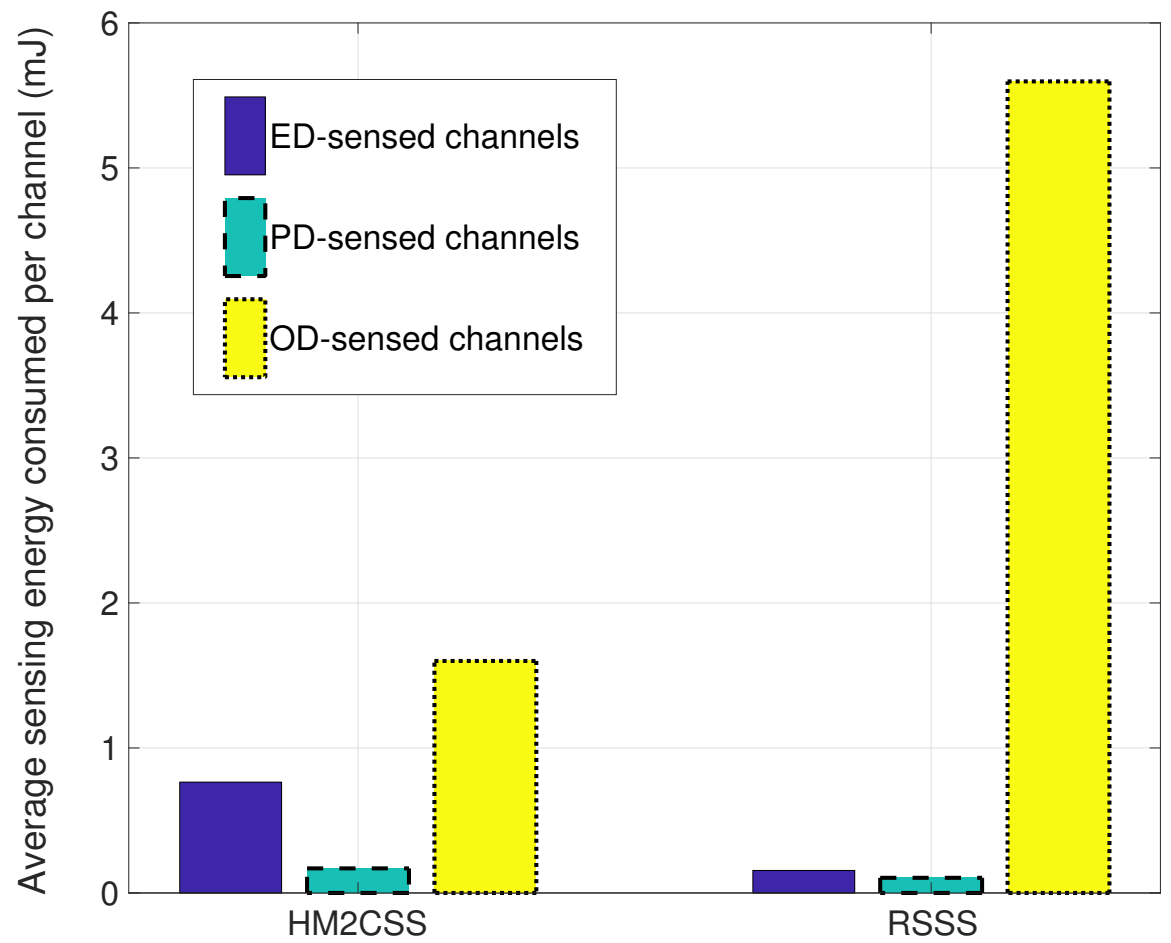

Figure 5.17: Comparison of proposed HM2CSS with existing RSSS in terms of average sensing energy consumption level across similar channel types.

scheme consumes most of the energy on sensing one channel and less on another. This is done through selecting near-equal, depending on availability, number of SUs to sense a certain channel. However, the existing RSSS scheme selects the number of SUs to sense a certain channel arbitrarily, i.e., in a random manner. This is why we observe approximately same level of energy consumed on sensing all channels by similar detector types for the proposed HM2CSS scheme, whereas in case of the RSSS scheme, energy consumed on the sensing process for different channels varies. It is essential to note that variation in the amount of energy being consumed for sensing channels 1 to 20, 21 to 40, and 41 to 50 comes from differences in the amount of samples being used for certain detector types. The higher the number of samples is being used to sense a certain channel, the higher the amount of energy is needed to perform the sensing process. Despite the fact that in total, the average amount of energy needed to be consumed on the sensing process for ED-sensed channels is higher for the HM2CSS scheme than the RSSS scheme and PD-sensed channels has a slight increase in the energy consumption as well, OD-sensed channels have drastically 


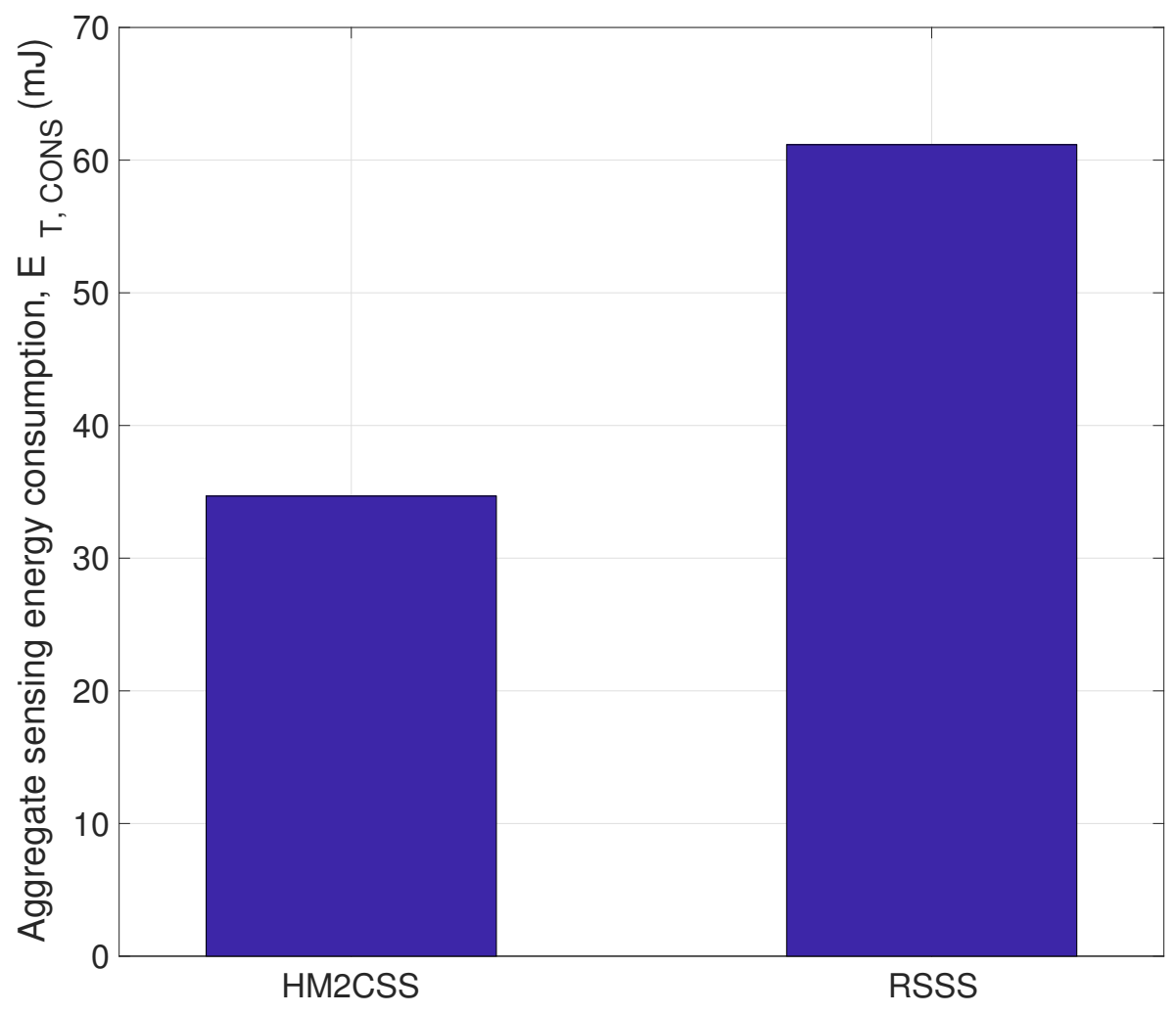

Figure 5.18: Aggregate energy consumption level on spectrum sensing for proposed HM2CSS and existing RSSS.

reduced energy consumption on sensing as shown in Fig. 5.17. This can be explained in the way that the more information is available about the channel, the less number of cooperative SUs is chosen to sense it. Finally, Fig. 5.18 illustrates that in total, the aggregate amount of energy needed to be consumed on the sensing process for the proposed HM2CSS scheme is $41.7 \%$ less than the existing RSSS scheme. This brings reduced aggregate energy consumption for the system configuration chosen. However, the aggregate consumption highly depends on the availability of the prior information about channels for both proposed and existing schemes.

\subsection{Summary}

In this chapter, we proposed a novel diffusion learning-based HM2CSS scheme for distributed multi-band CRNs. The proposed HM2CSS scheme consists of two stages. 
We formulated optimization problems in order to provide an efficient way of selecting cooperative SUs for all channels. The first stage consists of selecting a SU-leader based on which cooperative SUs can be selected in the second stage. Simulation results show that the proposed HM2CSS scheme brings improvements in terms of global detection probability and CRN throughput results. While the existing distributed scheme's performance starts degrading as soon as the network size increases, HM2CSS is proved to enhance the performance as the system size grows. This indicates HM2CSS as a scalable scheme in terms of detection performance and allows the network size grow by adding supplementary SUs without extra human intervention needed to keep system performance level stable. 


\section{Chapter 6}

\section{Conclusions and Future Work}

\subsection{Conclusions}

Due to the IoT emerge, distributed learning-based multi-band multi-user cognitive IoT systems are believed to be a part of future communication. However, lack of centralized control together with the increase in number of devices and dynamic environments place room for lots of challenges.

At first, we have discussed three different cognitive IoT system architectures, named as centralized, distributed, and hybrid. We pointed out advantages and disadvantages of each one of them. In total, cognitive IoT systems are believed to be hybrid, which include both centralized and distributed networks. However, due to the distributed nature of IoT and the focus placed on learning and feedback, distributed systems are of great importance. We have provided a list of challenges to be addressed in designing multi-band CSS schemes for cognitive IoT systems compared to conventional CSS schemes. These include application-centric approach, mobility of users, scalability, high integrity and heterogeneity, cooperation and learning, maintaining EE, fairness and cooperative SU-selection, computational complexity, sensing duration and synchronization, and interference management. The description of each one of them has been discussed in details.

In addition to this, we have examined the abilities of three different learningbased approaches to be incorporated in distributed cognitive IoT systems, named as incremental, consensus, and diffusion. The comparison has been provided, pointing out pros and cons of each one of them. Simulation results have been performed in terms of learning curves for the cases of no link failure and $30 \%$ link failure and the amount of iterations needed as the number of cooperative SUs grows. It has been 
proved that the diffusion-based learning algorithm is more efficient than the others mentioned above for cognitive IoT systems due to low complexity, relatively high speed of convergence, immunity to link failures, etc.

One of the main challenges in heterogeneous distributed learning-based CSS is cooperative SUs' scheduling to sense a subset of channels. To overcome this challenge, in this thesis, we propose a novel multi-band CSS scheme called HM2CSS. The proposed scheme is designed to solve the scalability in terms of detection performance, heterogeneity, fairness in cooperative SU-selection, sensing duration, and synchronization challenges of multi-band CSS in cognitive IoT systems. The proposed HM2CSS algorithm consists of two stages. The first stage involves selecting an SU-leader for each channel of the multi-band spectrum commodity. We formulate optimization problems depending on detector types used. Based on selected SU-leaders, cooperative SUs are selected in the second stage. For this purpose, an optimization problem is formulated. Then, cooperative SUs exchange locally sensed information using diffusion learning to determine the availability of channels. Simulation results show that the proposed HM2CSS scheme outperforms existing distributed and centralized schemes in terms of global detection probability and CRN throughput. The proposed HM2CSS scheme is proved to enhance system performance as the CRN size grows. This indicates the proposed HM2CSS scheme as scalable in terms of detection performance. It allows adding supplementary SUs without additional human intervention of bringing the performance level back. The proposed HM2CSS scheme satisfies the IEEE 802.22 detection performance standard, provides heterogeneity, and high performance in terms of global detection probability and CRN throughput for distributed cognitive IoT systems. The proposed HM2CSS scheme provides fair energy consumption for CSS on all channels and illustrates lower aggregate energy consumption for the system in total. Finally, one of the possible applications for the proposed scheme is smart transportations. Electrical vehicles can be equipped with sensing technologies and small computers, which are capable of performing the presented optimization problems. Nevertheless, HM2CSS has its own drawbacks yet to overcome as higher computational complexity and power consumption per channel for certain detector types. The scheme may still represent challenges for small IoT devices. Thus, future research insights are given in the next section in order to improve and overcome the drawbacks of HM2CSS and existing multi-band CSS schemes. 


\subsection{Future Work}

The research on the distributed CSS has mostly been limited to small scale networks. This thesis provides a unified problem formulation of distributed multi-band CSS to address challenges of cognitive IoT systems. The insights attained in this open demanding future research direction, including, but not limited to are:

- We can consider involving and comparing different compressive spectrum sensing and multi-co-set spectrum sensing techniques in order to decrease individual $\mathrm{SU}$ as well as system's sensing energy consumption in total.

- We have demonstrated that for the proposed HM2CSS scheme, there exists a maximum number of channels to be sensed by each SU, which illustrates the optimal systems performance. For example, in this thesis we did not have to sense eight channels per each SU as sensing five channels corresponded to similar detection performance. However, this was determined by means of simulations. In future, we are going to optimize the number of channels being sensed by each SU subject to the system performance analytically.

- In this thesis, we have assumed that each SU has sufficient amount of energy to perform diffusion-based learning. In future works, we are planning to investigate the case, when a SU can switch between diffusion and consensus-based learning to reduce the energy consumption of the system.

- Incorporating machine learning techniques to the learning process is another interesting topic of investigation. Machine learning can help in selecting cooperative SUs based on predictions and analytics.

- In this thesis, we have considered network-based reconfiguration, when each SU owns one detector. However, for some IoT applications owning strong SUs, which are able to have multiple CR units with different detector types, devicebased reconfiguration can be considered.

- This thesis considers opportunistic spectrum access in cognitive IoT systems. Other CR techniques can be considered for cognitive IoT systems as well. For instance, the underlay CR paradigm, where secondary IoT-nodes and PUs are allowed to share spectrum concurrently with interference constraints placed on secondary IoT-nodes. We also encourage the analysis of the overlay CR 
mechanism, where secondary IoT-nodes share spectrum with PUs concurrently by means of helping PU-transmitters to deliver the information to PU-receivers, i.e., acting as relays.

- Incorporating radio frequency EH (RFEH) techniques in order to increase the energy efficiency of the system is another approach to be considered. In case a $\mathrm{SU}$ is in the waiting mode or is not allowed to transmit, it could switch to the RFEH mode instead. This may further reduce human intervention as it can decrease the number of battery replacements in secondary-IoT nodes.

- This thesis highlights the importance of learning algorithms due to the distributed IoT nature. However, we as well mention that IoT networks will have a mixed topology of hybrid type. This is why investigating the borders of centralized and distributed systems, i.e., parts where the two different network topologies meet, is an important research topic to be considered.

- As discussed in this thesis, due to the distributed nature of IoT systems, the centralized network topology offer several disadvantages to IoT. Another way of overcoming them is by applying drone base stations as mobile cluster heads [61]. The analysis of hybrid network topologies with mobile drone base stations acting as cluster heads can be an interesting investigation.

- In hybrid IoT systems, when a FC fails, one of the SU nodes can take its role. In this case the chosen SU node is selected only if it is capable of performing FC tasks. An example of such systems is smart grid networks [62]. The problem may be set in the following way: given a network of 100 SUs, 10 from which are super-nodes, i.e., have the capabilities of performing FC tasks, what is the best way of choosing the new FC. In this case, at each time instance $T_{\text {instance }}$, the best super-node is chosen to perform FC tasks.

- One of the most important elements associated with distributed IoT systems is the ability to deal with local sensing. Locality is very important in IoT. However, it is not well-explored yet. Geo-location and stochastic geometry techniques can be combined together with the proposed scheme to increase system performance [63].

- In distributed cognitive IoT systems, it is essential to analyze time duration, when cooperative SU-selection has to be repeated. This problem is still open 
for research explorations and may depend on various factors, such as mobility of SUs, environmental changes, etc.

- Finally, it is important to investigate the response of the proposed algorithm to system changes. Currently addition of new users to the system requires the cooperative SU-selection process to be repeated over again. However, there may be more efficient solutions to this. The environment can be investigated together with the properties of new users and hence, decisions made whether it is better to repeat the process again or simply allow users join several sensing clusters. 


\section{List of References}

[1] M. A. Shah, S. Zhang, and C. Maple, "Cognitive radio networks for internet of things: Applications, challenges, and future," in 19th International Conference on Automation and Computing, Sep. 2013, pp. 1-6.

[2] X. Liu and J. L. Xie, "Priority-based spectrum access in cognitive D2D networks for IoT," in IEEE International Conference on Communications (ICC), May 2017, pp. 1-6.

[3] A. A. Khan, M. H. Rehmani, and A. Rachedi, "Cognitive-radio-based internet of things: Applications, architectures, spectrum related functionalities, and future research directions," IEEE Wireless Communications, vol. 24, no. 3, pp. 17-25, Jun. 2017.

[4] S. Rajendran, B. V. den Bergh, T. Vermeulen, and S. Pollin, "IEEE 5G spectrum sharing challenge: A practical evaluation of learning and feedback," IEEE Communications Magazine, vol. 54, no. 11, pp. 210-216, Nov. 2016.

[5] M. Ibnkahla, Cooperative Cognitive Radio Networks: The Complete Spectrum Cycle. Taylor and Francis Publishers, CRC Press, Boca Raton, FL, USA, 2015.

[6] A. El-Mougy, M. Ibnkahla, G. Hattab, and W. Ejaz, "Reconfigurable wireless networks," Proceedings of the IEEE, vol. 103, no. 7, pp. 1125-1158, Jul. 2015.

[7] S. Borkar and H. Pande, "Application of 5G next generation network to internet of things," in International Conference on Internet of Things and Applications (IOTA), Jan. 2016, pp. 443-447.

[8] G. Hattab and M. Ibnkahla, "Multiband spectrum access: Great promises for future cognitive radio networks," Proceedings of the IEEE, vol. 102, no. 3, pp. 282-306, Mar. 2014.

[9] S. Gaur and A. Annamalai, "Some integrals involving the $Q_{m}(a \sqrt{x}, b \sqrt{x})$ with application to error probability analysis of diversity receivers," IEEE Transactions on Vehicular Technology, vol. 52, no. 6, pp. 1568-1575, Nov. 2003.

[10] G. Fortino, "Agents meet the IoT: Toward ecosystems of networked smart objects," IEEE Systems, Man, and Cybernetics Magazine, vol. 2, no. 2, pp. 43-47, Apr. 2016. 
[11] R. Sharma, "Internet of things: An approach for advancement in educational institution," in 1st India International Conference on Information Processing (IICIP), Aug. 2016, pp. 1-4.

[12] C. Yang, J. Li, M. Guizani, A. Anpalagan, and M. Elkashlan, "Advanced spectrum sharing in 5G cognitive heterogeneous networks," IEEE Wireless Communications, vol. 23, no. 2, pp. 94-101, Apr. 2016.

[13] P. Rawat, K. Singh, and J. Bonnin, "Cognitive radio for M2M and internet of things: A survey," Computer Communications, vol. 94, pp. 1 - 29, Nov. 2016.

[14] K. B. Letaief and W. Zhang, "Cooperative communications for cognitive radio networks," Proceedings of the IEEE, vol. 97, no. 5, pp. 878-893, May 2009.

[15] P. Paysarvi-Hoseini and N. C. Beaulieu, "On the benefits of multichannel/wideband spectrum sensing with non-uniform channel sensing durations for cognitive radio networks," IEEE Transactions on Communications, vol. 60, no. 9, pp. 2434-2443, Sep. 2012.

[16] Z. Quan, S. Cui, A. H. Sayed, and H. V. Poor, "Optimal multiband joint detection for spectrum sensing in cognitive radio networks," IEEE Transactions on Signal Processing, vol. 57, no. 3, pp. 1128-1140, Mar. 2009.

[17] Z. Khan, J. J. Lehtomaki, S. I. Iellamo, R. Vuohtoniemi, E. Hossain, and Z. Han, "IoT connectivity in radar bands: A shared access model based on spectrum measurements," IEEE Communications Magazine, vol. 55, no. 2, pp. 88-96, Feb. 2017.

[18] M. McHenry, E. Livsics, T. Nguyen, and N. Majumdar, "XG dynamic spectrum access field test results [topics in radio communications]," IEEE Communications Magazine, vol. 45, no. 6, pp. 51-57, Jun. 2007.

[19] N. Khambekar, V. Chaudhary, and C. Spooner, "Estimating the use of spectrum for defining and enforcing the spectrum access rights," in IEEE Military Communications Conference (MILCOM), Oct. 2015, pp. 250-257.

[20] J. Mitola and G. Q. Maguire, "Cognitive radio: Making software radios more personal," IEEE Personal Communications, vol. 6, no. 4, pp. 13-18, Aug. 1999.

[21] S. Lin, L. Kong, Q. Gao, M. K. Khan, Z. Zhong, X. Jin, and P. Zeng, "Advanced dynamic channel access strategy in spectrum sharing $5 \mathrm{G}$ systems," IEEE Wireless Communications, vol. 24, no. 5, pp. 74-80, Oct. 2017.

[22] S. Haykin, "Cognitive radio: Brain-empowered wireless communications," IEEE Journal on Selected Areas in Communications, vol. 23, no. 2, pp. 201-220, Feb. 2005.

[23] Y. C. Liang, K. C. Chen, G. Y. Li, and P. Mahonen, "Cognitive radio networking and communications: An overview," IEEE Transactions on Vehicular Technology, vol. 60, no. 7, pp. 3386-3407, Sep. 2011. 
[24] B. Suseela and D. Sivakumar, "Non-cooperative spectrum sensing techniques in cognitive radio - a survey," in IEEE Technological Innovation in ICT for Agriculture and Rural Development (TIAR), Jul. 2015, pp. 127-133.

[25] P. Rungsawang and A. Khawne, "The implementation of spectrum sensing and spectrum allocation on cognitive radio," in 19th International Conference on Advanced Communication Technology (ICACT), Feb. 2017, pp. 817-821.

[26] R. F. Shigueta, M. Fonseca, A. C. Viana, A. Ziviani, and A. Munaretto, "A strategy for opportunistic cognitive channel allocation in wireless internet of things," in IFIP Wireless Days (WD), Nov. 2014, pp. 1-3.

[27] I. F. Akyildiz, W. y. Lee, M. C. Vuran, and S. Mohanty, "A survey on spectrum management in cognitive radio networks," IEEE Communications Magazine, vol. 46, no. 4, pp. 40-48, Apr. 2008.

[28] H. B. Salameh, S. Almajali, M. Ayyash, and H. Elgala, "Security-aware channel assignment in IoT-based cognitive radio networks for time-critical applications," in Fourth International Conference on Software Defined Systems (SDS), May 2017, pp. 43-47.

[29] X. Kang, Y. C. Liang, and J. Yang, "Riding on the primary: A new spectrum sharing paradigm for wireless-powered IoT devices," in IEEE International Conference on Communications (ICC), May 2017, pp. 1-6.

[30] M. Eisenhauer, S. Guilln, P. Sala, G. Fico, M. Arredondo, A. Cano, J. Posada, G. Gutirrez, C. Palau, K. Votis, C. Verdouw, S. Wolfert, G. Beers, H. Sundmaeker, G. Chatzikostas, S. Ziegler, C. Hemmens, M. Holst, A. Sthlbrst, and O. Lenz, "IoT european large-scale pilots: Integration, experimentation, and testing," in Cognitive Hyperconnected Digital Transformation - Internet of Things Intelligence Evolution, Jun. 2017, pp. 221-282.

[31] H2020, "Horizon 2020 work programme 2018-2020 - strategic programme overarching document," http://ec.europa.eu/programmes/horizon2020/sites/ horizon2020/files/stratprog_overarching_version_for_publication.pdf, Oct. 2017, Accessed on 15 Dec. 2017.

[32] F. Meucci, O. Cabral, F. J. Velez, A. Mihovska, and N. R. Prasad, "Spectrum aggregation with multi-band user allocation over two frequency bands," in IEEE Mobile WiMAX Symposium, Jul. 2009, pp. 81-86.

[33] R. Zhu, X. Zhang, X. Liu, W. Shu, T. Mao, and B. Jalaian, "ERDT: Energyefficient reliable decision transmission for intelligent cooperative spectrum sensing in industrial IoT," IEEE Access, vol. 3, pp. 2366-2378, Nov. 2015.

[34] A. Ebrahimzadeh, M. Najimi, S. M. H. Andargoli, and A. Fallahi, "Sensor selection and optimal energy detection threshold for efficient cooperative spectrum sensing," IEEE Transactions on Vehicular Technology, vol. 64, no. 4, pp. 15651577, Apr. 2015. 
[35] Y. Abdi and T. Ristaniemi, "Optimal multiband cooperative spectrum sensing with random interruptions," in IEEE Global Communications Conference (GLOBECOM), Dec. 2016, pp. 1-7.

[36] T. Xiong, Z. Li, Y. D. Yao, and P. Qi, "Random, persistent, and adaptive spectrum sensing strategies for multi-band spectrum sensing in cognitive radio networks with secondary user hardware limitation," IEEE Access, vol. 5, pp. 1485414866, Aug. 2017.

[37] W. Ejaz, G. Hattab, N. Cherif, M. Ibnkahla, F. Abdelkefi, and M. Siala, "Cooperative spectrum sensing with heterogeneous devices: Hard combining versus soft combining," IEEE Systems Journal, vol. 12, no. 1, pp. 1-12, Jul. 2016.

[38] F. Zhou, Z. Li, J. Si, B. Hao, and P. Qi, "Adaptive secondary-user selection without prior information for cooperative spectrum sensing in CRNs," in International Conference on Computer, Information and Telecommunication Systems (CITS), Jul. 2015, pp. 1-5.

[39] A. Li, G. Han, L. Shu, and M. Guizani, "Cooperative secondary users selection in cognitive radio ad hoc networks," in International Wireless Communications and Mobile Computing Conference (IWCMC), Sep. 2016, pp. 915-920.

[40] X. R. Li and X.-B. Li, "Common fallacies in applying hypothesis testing," in 11th International Conference on Information Fusion, Jun. 2008, pp. 1-8.

[41] G. Hattab and M. Ibnkahla, "Enhanced pilot-based spectrum sensing algorithm," in 27th Biennial Symposium on Communications (QBSC), Jun. 2014, pp. 57-60.

[42] E. Axell and E. G. Larsson, "Optimal and sub-optimal spectrum sensing of OFDM signals in known and unknown noise variance," IEEE Journal on Selected Areas in Communications, vol. 29, no. 2, pp. 290-304, Feb. 2011.

[43] Y. C. Liang, Y. Zeng, E. C. Y. Peh, and A. T. Hoang, "Sensing-throughput tradeoff for cognitive radio networks," IEEE Transactions on Wireless Communications, vol. 7, no. 4, pp. 1326-1337, Apr. 2008.

[44] S. Chaudhari, V. Koivunen, and H. V. Poor, "Autocorrelation-based decentralized sequential detection of OFDM signals in cognitive radios," IEEE Transactions on Signal Processing, vol. 57, no. 7, pp. 2690-2700, Jul. 2009.

[45] M. Souryal, M. Ranganathan, J. Mink, and N. E. Ouni, "Real-time centralized spectrum monitoring: Feasibility, architecture, and latency," in IEEE International Symposium on Dynamic Spectrum Access Networks (DySPAN), Sep. 2015, pp. 106-112.

[46] Y. Teranishi, R. Banno, and T. Akiyama, "Scalable and locality-aware distributed topic-based pub/sub messaging for IoT," in IEEE Global Communications Conference (GLOBECOM), Dec. 2015, pp. 1-7.

[47] H. Zhang, D. Jiang, F. Li, K. Liu, H. Song, and H. Dai, "Cluster-based resource allocation for spectrum-sharing femtocell networks," IEEE Access, vol. 4, pp. 8643-8656, Dec. 2016. 
[48] S. P. Eswaran and J. Bapat, "Service centric markov based spectrum sharing for internet of things (IoT)," in IEEE Region 10 Symposium, May 2015, pp. 9-12.

[49] T. Park, N. Abuzainab, and W. Saad, "Learning how to communicate in the internet of things: Finite resources and heterogeneity," IEEE Access, vol. 4, pp. 7063-7073, Nov. 2016.

[50] C. Pham, Y. Lim, and Y. Tan, "Management architecture for heterogeneous IoT devices in home network," in IEEE 5th Global Conference on Consumer Electronics, Oct. 2016, pp. 1-5.

[51] A. H. Sayed, "Adaptive networks," Proceedings of the IEEE, vol. 102, no. 4, pp. 460-497, Apr. 2014.

[52] Z. Li, F. R. Yu, and M. Huang, "A distributed consensus-based cooperative spectrum-sensing scheme in cognitive radios," IEEE Transactions on Vehicular Technology, vol. 59, no. 1, pp. 383-393, Jan. 2010.

[53] A. Hajihoseini and S. A. Ghorashi, "Distributed spectrum sensing for cognitive radio sensor networks using diffusion adaptation," IEEE Sensors Letters, vol. 1, no. 5, pp. 1-4, Oct. 2017.

[54] A. Ercan, O. Sunay, and I. F. Akyildiz, "RF energy harvesting and transfer for spectrum sharing cellular IoT communications in 5G systems," IEEE Transactions on Mobile Computing, vol. PP, no. 99, pp. 1-1, Aug. 2017.

[55] R. Narayanan, M. Srinivasan, S. A. Karthikeya, and C. S. R. Murthy, "A novel fairness-driven approach for heterogeneous gateways' link scheduling in IoT networks," in IEEE International Conference on Communications (ICC), May 2017, pp. 1-7.

[56] M. Soltani, T. Baykas, and H. Arslan, "On reducing multiband spectrum sensing duration for cognitive radio networks," in 84th Vehicular Technology Conference (VTC-Fall), Sep. 2016, pp. 1-5.

[57] N. Thakoor and J. Gao, "Branch-and-bound for model selection and its computational complexity," IEEE Transactions on Knowledge and Data Engineering, vol. 23, no. 5, pp. 655-668, May 2011.

[58] W. Ejaz and M. Ibnkahla, "Multiband spectrum sensing and resource allocation for IoT in cognitive 5G networks," IEEE Internet of Things Journal, vol. 5, no. 1, pp. 150-163, Feb. 2018.

[59] H. N. Abdullah and H. S. Abed, "Improvement of energy consumption in cognitive radio by reducing the number of sensed samples," in Al-Sadeq International Conference on Multidisciplinary in IT and Communication Science and Applications (AIC-MITCSA), May 2016, pp. 1-6.

[60] J. Vartiainen, M. Hyhty, R. Vuohtoniemi, and V. L. Ramani, "The future of spectrum sensing," in Eighth International Conference on Ubiquitous and Future Networks (ICUFN), Jul. 2016, pp. 247-252. 
[61] I. Bor-Yaliniz and H. Yanikomeroglu, "The new frontier in RAN heterogeneity: Multi-tier drone-cells," IEEE Communications Magazine, vol. 54, no. 11, pp. 48-55, Nov. 2016.

[62] L. Zhu, M. Li, Z. Zhang, X. Du, and M. Guizani, "Big data mining of users' energy consumption patterns in the wireless smart grid," IEEE Wireless Communications, vol. 25, no. 1, pp. 84-89, Feb. 2018.

[63] F. J. Martin-Vega, B. Soret, M. Aguayo-Torres, I. Z. Kovacs, and G. Gomez, "Geo-location based access for vehicular communications: Analysis and optimization via stochastic geometry," IEEE Transactions on Vehicular Technology, vol. PP, no. 99, pp. 1-1, Nov. 2017. 